SANDIA REPORT

SAND96-1960 • UC-721

Unlimited Release

Printed January 1997 Feo 1439

\title{
0 TI
}

\section{Condensed Listing of Surface Boreholes at the Waste Isolation Pilot Plant Project through 31 December 1995}

Leslie R. Hill, Richard Aguilar, Jerry W. Mercer, Gretchen Newman

\section{Prepared by}

Sandia National Laboratories

Albuquerque, New Mexico 87185 and Livermore, California 94550

for the United States Department of Energy

under Contract DE-AC04-94AL85000

Approved for public release; distribution is unlimited.




Issued by Sandia National Laboratories, operated for the United States Department of Energy by Sandia Corporation.

NOTICE: This report was prepared as an account of work sponsored by an agency of the United States Government. Neither the United States Government nor any agency thereof, nor any of their employees, nor any of their contractors, subcontractors, or their employees, makes any warranty, express or implied, or assumes any legal liability or responsibility for the accuracy, completeness, or usefulness of any information, apparatus, product, or process disclosed, or represents that its use would not infringe privately owned rights. Reference herein to any specific commercial product, process, or service by trade name, trademark, manufacturer, or otherwise, does not necessarily constitute or imply its endorsement, recommendation, or favoring by the United States Government, any agency thereof or any of their contractors or subcontractors. The views and opinions expressed herein do not necessarily state or reflect those of the United States Government, any agency thereof or any of their contractors.

Printed in the United States of America. This report has been reproduced directly from the best available copy.

Available to DOE and DOE contractors from

Office of Scientific and Technical Information

PO Box 62

Oak Ridge, TN 37831

Prices available from (615) 576-8401, FTS 626-8401

Available to the public from

National Technical Information Service

US Department of Commerce

5285 Port Royal Rd

Springfield, VA 22161

NTIS price codes

Printed copy: A05

Microfiche copy: A01 
DISCLAIMER

Portions of this document may be illegible in electronic image products. Images are produced from the best available original document. 


\title{
Condensed Listing of Surface Boreholes at the Waste Isolation Pilot Plant Project through 31 December 1995
}

\author{
Leslie R. Hill \\ Compliance Support Department \\ Richard Aguilar \\ YMP System Performance Assessment Department \\ Jerry W. Mercer \\ Engineering Projects and Explosives Applications Department \\ Sandia National Laboratories \\ Albuquerque, NM 87185-1341 \\ Gretchen Newman \\ GRAM, Inc. \\ Albuquerque, NM 87110
}

\begin{abstract}
This report contains a condensed listing of Waste Isolation Pilot Plant (WIPP) project surface boreholes drilled for the purpose of site selection and characterization through 31 December 1995. The US Department of Energy (DOE) sponsored the drilling activities, which were conducted primarily by Sandia National Laboratories. The listing provides physical attributes such as location (township, range, section, and state-plane coordinates), elevation, and total borehole depth, as well as the purpose for the borehole, drilling dates, and information about extracted cores. The report also presents the hole status (plugged, testing, monitoring, etc.) and includes salient findings and references. Maps with borehole locations and times-of-drilling charts are included.
\end{abstract}




\section{ACKNOWLEDGMENTS}

The authors would like to acknowledge the following individuals who contributed to the completion of this report. Richard L. Beauheim of Organization 6115 provided valuable input during our compilation of the WIPP site surface borehole data; he answered many technical questions arising during this exercise and directed the authors to existing data reports on specific boreholes. Steven J. Lambert gave detailed review guidance that was invaluable to the successful production of the report. Dennis W. Powers, Sue-Ellen Shaffer Osika, Robert D. Statler, and Paul E. Sanchez reviewed the draft report and provided input on the completeness and technical accuracy of the borehole data. Bob Jones of Tech Reps, Inc. provided comprehensive assistance in developing the graphics. Tim Peterson and Molly Minahan of Tech Reps, Inc. gave timely and effective editorial assistance. The authors would also like to acknowledge P.D. Seward for the initial compilation of WIPP site surface borehole data up to 30 September 1981, reported in SAND82-0080 (Seward, 1982). The present report updates information in his report and provides a common frame of reference for surface borehole spatial and physical data obtained during the characterization of the WIPP site through 1995. 


\section{PREFACE}

The data presented in this report were obtained primarily from published basic data reports and other Sandia-sponsored publications. The authors attempted to ensure the accuracy of the data reported herein; however, they. do not assume responsibility for errors in previously published documents that are discussed here. Where discrepancies were encountered in data reported among the various data sources, the authors identified the source of these discrepancies, often examining original field data sheets and dayfiles to verify the data. Corrections and/or changes in previously published data are noted by footnotes on the spreadsheets. The principal data sources for this report were the published Sandia basic data reports for the various boreholes and hydropads, USGS-OFR 78-592 (Jones, 1978) for potash holes (P-1 through P-21), and SAND88-1065 (Gonzales, 1989) for borehole locations. 
Page intentionally left blank. 


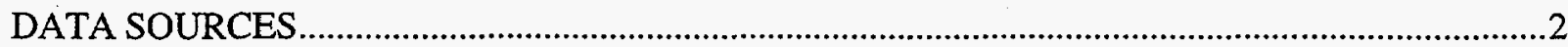

BOREHOLE DATA



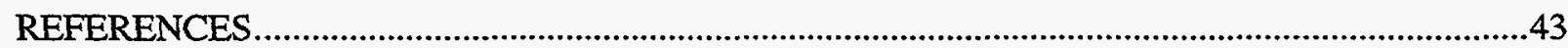

APPENDIX A: MEMORANDA REGARDING REFERENCED DATA .......................................

APPENDIX B: AS-BUILT ELEMENTS OF THE FOUR WIPP SHAFTS ......................................

\section{Table}

Table 1. DOE-Sponsored Surface Boreholes at the Waste Isolation Pilot Plant.

.6

\section{Figures}

Figure 1. Area of the Waste Isolation Pilot Plant-regional scale. .36

Figure 2. Area of the Waste Isolation Pilot Plant—immediate boundary scale................................37

Figure 3. Area of the Waste Isolation Pilot Plant $\longrightarrow$ underground repository scale. ...............................38

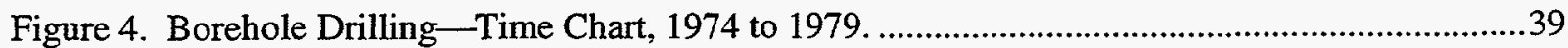

Figure 5. Borehole Drilling — Time Chart, 1979 to 1985 ................................................................40

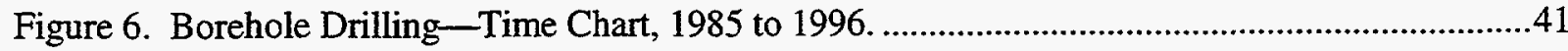


Page intentionally left blank. 


\section{BACKGROUND}

The history of radioactive waste disposal programs in the United States, which led to development of the Waste Isolation Pilot Plant (WIPP) project in southeastern New Mexico, began in the 1950s. In 1957 the National Academy of Sciences selected salt as an appropriate medium for a nuclear waste repository (NAS, 1957). Among the reasons for the selection were: (1) salt deposits demonstrate isolation from circulating groundwaters for long periods of geologic time; (2) salt deformation occurs slowly over time and would therefore prevent the formation of fractures or heal openings that could otherwise allow the release of radioactivity to the biosphere; and (3) salt readily dissipates heat generated from nuclear wastes.

In 1974 the Atomic Energy Commission authorized a detailed evaluation of an area with extensive geologic salt deposits in the Delaware Basin of southeastern New Mexico, approximately 50 $\mathrm{km}$ east of Carlsbad. Results of the evaluation indicated that the area would be suitable for a nuclear waste repository (Powers et al., 1978). The area had low population density (fewer than 30 inhabitants within $16 \mathrm{~km}$ of the site), and land acquisition would be simplified by maximum use of federal, rather than state and private, land. The proposed site was evaluated for its suitability as a nuclear waste repository through extensive investigations that included exploratory drilling, hydrologic testing, and the use of geophysical techniques. In most cases, the exploratory boreholes had multiple purposes to aid in establishing the geologic and hydrologic characteristics of the WIPP site. The host medium selected for the underground repository was the Permian Age Salado Formation, composed of thick (more than $600-\mathrm{m}$ ) bedded salts. The repository was to be located about $656 \mathrm{~m}(2,150 \mathrm{ft})$ below the ground surface.

In 1976 the project was given its present name, and in 1979 the WIPP was established by the US Department of Energy (DOE) as a research and development facility for the purpose of demonstrating the safe geologic disposal of transuranic radioactive wastes resulting from our nation's defense activities. Specifically, the objective of the WIPP project was to address the technology associated with long-term isolation of defense-related nuclear wastes in the geologic medium of natural salt deposits. Public Law 96-164 (1979) authorized the DOE to proceed with the development of the WIPP facility.

Prior to October 1992, the land of the WIPP site was administered by the US Department of Interior, Bureau of Land Management. The WIPP site encompasses 4,145 hectares (10,240 acres); surrounding areas are used primarily for livestock grazing, some potash mining, and oil and gas production (US DOE, 1980). Congress transferred jurisdiction of the WIPP site to the Secretary of Energy on 30 October 1992, through the Waste Isolation Pilot Plant Land Withdrawal Act (LWA) of 1992 (Public Law 102-579).

The agency now known as the DOE has undergone three name changes since its inception as the Atomic Energy Commission in 1946; thus many of the WIPP boreholes were named in accordance with the current designation of the responsible agency at the time of drilling (e.g., AEC: Atomic Energy Commission, ERDA: Energy Research and Development Administration). Two of the exploratory boreholes, AEC 7 and AEC 8, were drilled by Oak Ridge National Laboratory in 1974, before Sandia National Laboratories assumed responsibility for the site characterization activities. AEC 7 and AEC 8 were eventually deepened for further evaluation. Borehole Cabin Baby \#1 was acquired by DOE from a petroleum company and borehole D-268 was drilled by a potash mining company. These two boreholes were used by Sandia in site characterization work and thus are included in this report. The AEC, ERDA, DOE, and WIPP holes were drilled primarily to characterize the geology and stratigraphy of the WIPP site and its environs and to investigate the presence or absence of various geologic features or processes such as faulting, anticlinal structures, breccia pipes, and salt dissolution in the rocks near the proposed repository. The potash resource 
evaluation boreholes (P-1 through P-21) were drilled under the management of Fenix \& Scisson, Inc. These boreholes were logged and the stratigraphy and core were described by the US Geological Survey (USGS) Special Projects Division for DOE/Sandia to expand knowledge on the distribution, composition, and tonnage of potash resources in the WIPP site area (Jones, 1978).

WIPP 17, 20,23, and 24 were planned but not drilled because the technical justifications for these holes were satisfied by data obtained from earlier boreholes. WIPP 25 through WIPP 30 were drilled specifically to define the geology and hydrology of the Nash Draw area, a broad, shallow topographic depression west of the WIPP site (Bachman, 1980).

Rock units comprising the Late Permian Rustler Formation overlying the Salado Formation were studied extensively because some units contain groundwater. The Rustler plays an integral role in the performance assessment of the WIPP. These rock units may influence the migration of radionuclides and gases released from the repository to the accessible environment in the event of inadvertent human intrusion. Many of the WIPP boreholes (vertical holes drilled from the ground surface), particularly $\mathrm{H}-1$ through $\mathrm{H}-19$, were drilled specifically to evaluate the hydrologic and geochemical properties of the Magenta and Culebra dolomite members of the Rustler Formation and the contact zone between the Rustler and Salado formations (borehole $\mathrm{H}-13$ was not drilled). Boreholes H-19(b0) through H-19(b7), completed in 1995, were drilled for hydrologic and tracerbased flow testing (US DOE, 1995a).

Studies over the past two decades have determined that the Culebra Dolomite is the most transmissive rock unit above the proposed WIPP repository; it is thus considered to be the most probable pathway for radionuclide transport to the accessible environment if the repository is ever breached (US DOE, 1994). As part of the WIPP Water Quality Sampling Program (US DOE, 1995b), boreholes WQSP-1 through WQSP-6 were drilled for water quality monitoring and tracer tests in the Culebra Dolomite, and WQSP-6a was drilled for water quality monitoring in the Dewey Lake Red Beds above the Rustler.

\section{DATA SOURCES}

This report documents drilling activities performed in support of the WIPP Project. The listing summarizes the DOE's surface borehole drilling and testing activities through 31 December 1995 in spreadsheet format. This condensed listing of borehole data could be combined with other relevant WIPP project information to support the development of a Geographic Information System (GIS) (Aguilar et al., 1995). The data collected have been interpreted and published in a variety of documents, including basic data reports, hydrologic/hydraulic testing reports, potash resource test drilling reports, and other DOE, US Geological Survey, Sandia, and private industry reports. Some of these reports are listed among the references. Basic data reports were generally written as soon as possible after hole completion by the geologist who "rode" the hole.

A significant portion of the information presented here was obtained from basic data reports. The report by Seward (1982), which is an abridged surface borehole history for the WIPP site that included boreholes drilled to 30 September 1981, provided motivation to update the database.

Compilation of borehole data for the site required extensive review of the literature coupled with personal contacts with Sandia WIPP personnel, Westinghouse Electric Corporation employees, and private contractors familiar with the history of surface borehole activities at the WIPP. The January 22, 1991 memo from J.W. Mercer of Sandia provided information regarding the current status of the boreholes (see Appendix A). The borehole data (Table 1) are followed by maps showing borehole locations within the WIPP complex and surrounding area (Figures 1, 2, and 3). The 16-section 
controlled area of the WIPP, designated by the 1992 LWA, is outlined in these maps. Figures 4, 5 , and 6 show the chronology of the drilling program.

\section{BOREHOLE DATA}

The following is a key describing the data presented in Table 1:

$\mathbf{W D}=$

Borehole designation. The holes listed in Table 1 are grouped into four sections: (1) geologic evaluation, (2) hydrologic testing and monitoring, (3) resource evaluation, and (4) shaft construction. Within each section the holes are listed by hole alphanumeric identification number, except for the four shafts, which are listed chronologically.

Letters used as subdesignations for the hydrologic boreholes are based on the initial target horizon to be tested; for example, "a" and "b" represent completion to the Magenta and Culebra members of the Rustler Formation, respectively, and "c" represents completion to the Rustler/Salado contact.

The format used in naming the hydrologic boreholes varied somewhat over the 21year period (1974-1995) because of individual labeling preferences by those responsible for the drilling activities. For example, multiple boreholes at a given hydropad were at times differentiated by uppercase letter subdesignations (e.g., H-4A, H-4B, H-4C). In contrast, boreholes at other hydropad sites were at times differentiated by the use of lowercase letters with or without parentheses; for example, $\mathrm{H}-2$ (b1) and $\mathrm{H}-2 \mathrm{(b2}$ ) are two neighboring holes on pad $\mathrm{H}-2$ drilled to the Culebra. In the case of the H-3 hydropad, a combination of these two labeling schemes resulted. The first three boreholes, $\mathrm{H}-3$ (b1), H-3 (b2) and H-3 (b3), were completed to the Culebra Dolomite, whereas H-3D was completed to the Dewey Lake Red Beds overlying the Rustler Formation. Additionally, the H-3D borehole was drilled later than the other $\mathrm{H}-3$ boreholes.

For consistency, the authors have chosen to use lower case letter designations without parentheses to differentiate among multiple hydropad boreholes.

\section{Drilling Start/End Date =}

Month/Day/Year surface borehole drilling commenced (spudded in) and Month/Day/Year drilling was completed. If spudded in and drill completion dates were unavailable, the start and completion dates of the borehole field operations were used.

\section{Purpose $=$}

Abbreviation of general purpose for surface borehole drilling.

BHP - Borehole plugging test.

BREC - Breccia pipe exploration hole, a subset of GEO.

GEO - Geologic evaluation hole.

GEOHYD - Geologic, hydrologic evaluation hole.

HYD - Hydrologic monitoring hole (measuring fluid level and taking samples).

STRAT - Stratigraphic hole.

MULTI - Borehole used for several different purposes (e.g., GEO, HYD, STRAT).

NDGEOHYD - Nash Draw geologic and hydrologic test holes.

POT - Potash resource assessment hole. 
POTHYD - Potash resource assessment hole subsequently converted to a hydrologic hole.

RCHYD - Recompleted hydrologic hole.

HYDROCARBON - Borehole drilled by private industry for oil and/or gas exploration.

ENG - Borehole drilled for engineering activities associated with development of the WIPP repository.

Status $=$

Current condition of the borehole as of this printing. For example, a borehole may be described as plugged or in use as a hydrologic monitoring hole. Wherever possible, casing information is included. Most of this information was extracted from the January 22, 1991 memo from J.W. Mercer to W.D. Weart entitled "Test Holes Drilled in Support of Geotechnical Studies at the WIPP Site" (see Appendix A).

\section{Township/Range/Section and Section X-Y Coordinates $=$}

Township, Range, Section, and Section X-Y coordinates of the borehole location measured perpendicular from designated section lines (in feet). Most data were obtained from basic data reports and the "As-Built Surveys" for the boreholes reported in Table 3-5 of Gonzales (1989). For example, $1001 \mathrm{FNL}, 50 \mathrm{FEL}$ signifies that the hole is located at the intersection 1001 feet perpendicular to north section line and 50 feet perpendicular to east section line.

\section{State-Plane X-Y Coordinates =}

Most of the state-plane coordinates presented in Table 1 are taken from Table 3-6 of Gonzales (1989). State-plane coordinates for holes drilled after 1989 were extracted from recent data reports. The state-plane coordinates for each borehole were tied to surveyed distances from section lines by using known coordinates of the nearest NE section corner. An angle correction of $0.34^{\circ}$ clockwise from the section line to the state coordinate system was used in the conversion because the two systems do not run parallel. Section corner coordinates were provided to Sandia by the USGS and are listed in Appendix D of Gonzales (1989).

The point of origin for the state-plane coordinate system of the New Mexico east grid zone is located at $31^{\circ} 00^{\prime} \mathrm{N}$ and $104^{\circ} 20^{\prime} \mathrm{W}$. Origin values are arbitrarily set at 500,000 feet for the $\mathrm{X}$ coordinate and 0 feet for the $\mathrm{Y}$ coordinate. For example, borehole $\mathrm{P}-13$, which is located 156,973 feet east and 509,039 feet north of the point of origin, has state-plane coordinates of $656,973 \mathrm{X}$ and $509,039 \mathrm{Y}$

Elevation $(\mathrm{ft})=$

Surface elevation in feet above mean sea level. Elevations were taken from basic data reports and Gonzales (1989). When surface elevations were unavailable, monument elevations were employed.

\section{Total Depth (Below Ground Surface) $(\mathrm{ft})=$}

Total borehole depth in feet, rounded off to nearest foot using ground surface as the reference base. For boreholes where total depth was reported in basic data reports or other reference sources as depth from the top of the Kelly Bushing (KB), we have corrected to ground level reference base by subtracting the $\mathrm{KB}$ height above the ground surface from the total depth. These boreholes are indicated by an asterisk (*) following the reported depth. The KB height is given when available. 
Depth of Cored Interval(s) and Core Diameter(s) =

Interval(s) within the boreholes where core-drilling equipment was used. Most cores listed in Table 1 are size NX ( $25 / 32$ in. [54.8 mm]). Core diameters are given in the table when available. In cases where multiple core diameters are listed, deeper cores sometimes have larger diameters than shallower cores because the borehole was reamed to a larger diameter during subsequent drilling operations.

\section{Reference(s) $=$}

Report number, author(s), and date of publications containing information about the borehole; basic data reports were a key source.

\section{Comments $=$}

Purpose and objectives for the borehole. A brief statement on findings is included when available. It should be noted that findings resulting from initial basic interpretations may be changed by subsequent investigations.

The names of some geologic units-particularly the lower member of the Rustler Formation and the Dewey Lake Formation-have varied in the WIPP literature over the years. The former has been called either the "unnamed lower member" or the "lower member" of the Rustler, and a recent proposal recommends that the unit be informally named the Los Medaños Member (Holt and Powers, 1988); for brevity, this report uses the term "lower member." Names for the latter unit have also varied through the years. This report follows early usage and refers to the unit as the "Dewey Lake Red Beds."

\section{UNITS OF MEASURE}

All measurements related to the boreholes are reported in English units. These units are used to facilitate the comparison of original measurements by surveyors to establish the geographic coordinates of the boreholes, by drillers reporting sample and core depths, and by geophysical loggers in recording inhole variations in rock properties versus depth. If metric equivalents of the English units are desired, the following conversion factors should be used.

$\begin{array}{cc}\text { Multiply English unit } & \text { by } \\ \text { foot } & 0.3048 \\ \text { inch } & 25.4 \\ \text { inch } & 2.54 \\ \text { mile } & 1.6093 \\ \text { acre } & 0.405\end{array}$

to obtain metric equivalent
meter
millimeter
centimeter
kilometer
hectare


Table 1. DOE-Sponsored Surface Boreholes at the Waste Isolation Pilot Plant (continued)

\begin{tabular}{|c|c|c|c|c|c|c|c|c|}
\hline ID & $\begin{array}{l}\text { Drilling } \\
\text { Start/End } \\
\text { Date }\end{array}$ & Purpose & Status & $\begin{array}{l}\text { Township/ } \\
\text { Range/ } \\
\text { Section }\end{array}$ & $\begin{array}{l}\text { Section } X \& Y \\
\text { Coordinates }\end{array}$ & $\begin{array}{l}\text { State- } \\
\text { Plane } \\
\text { Coord. } \\
X \text { (ft) }\end{array}$ & $\begin{array}{c}\text { State- } \\
\text { Plane } \\
\text { Coord. } \\
Y \text { (ft) }\end{array}$ & $\begin{array}{c}\text { Surface } \\
\text { Elevation } \\
\text { (ft) }\end{array}$ \\
\hline \multicolumn{9}{|c|}{ Geologic Evaluation } \\
\hline AEC 7 & $\begin{array}{l}03 / 19 / 74 \\
04 / 48 / 74\end{array}$ & MULTI & $\begin{array}{l}\text { Partially } \\
\text { plugged/standby } \\
\text { condition. }\end{array}$ & T21S, R32E, S31 & 2039 FNL, 2037 FEL & 691,829 & 523,133 & 3656 \\
\hline $\begin{array}{c}A E C 7 \\
\text { (deepened) }\end{array}$ & $\begin{array}{l}02 / 27 / 79- \\
05 / 06 / 79\end{array}$ & $\begin{array}{c}\text { RCHYD, } \\
\text { BHP }\end{array}$ & $\begin{array}{l}\text { Used as a } \\
\text { hydrologic } \\
\text { monitoring well. }\end{array}$ & T21S, R32E, S31 & $2039 \mathrm{FNL}, 2037 \mathrm{FEL}$ & 691,829 & 523,133 & 3656 \\
\hline AEC 8 & $\begin{array}{l}04 / 24 / 74- \\
05 / 19 / 74\end{array}$ & GEO & $\begin{array}{l}\text { Partially } \\
\text { plugged/standby } \\
\text { condition. }\end{array}$ & T22S, R31E, S11 & 935 FNL, 1979 FWL & 679,951 & 513,567 & 3532 \\
\hline $\begin{array}{c}\text { AEC } 8 \\
\text { (deepened) }\end{array}$ & $\begin{array}{l}06 / 28 / 76- \\
08 / 05 / 76\end{array}$ & RCHYD & $\begin{array}{l}\text { Used as a } \\
\text { hydrologic } \\
\text { monitoring well. }\end{array}$ & T22S, R31E, S11 & $935 \mathrm{FNL}, 1979 \mathrm{FW}$ & 679,951 & 513,567 & 3532 \\
\hline $\begin{array}{c}\text { B-25 } \\
\text { (BECHTEL 25) }\end{array}$ & $\begin{array}{l}12 / 01 / 78- \\
01 / 05 / 79\end{array}$ & $\begin{array}{c}\text { ENG, HYD, } \\
\text { STRAT }\end{array}$ & Plugged & T22S, R31E, S20 & 796 FSL, 777 FEL & 666,693 & 499,415 & 3408 \\
\hline$D O E-1$ & $\begin{array}{l}07 / 14 / 82- \\
07 / 28 / 82\end{array}$ & $\begin{array}{l}\text { STRAT, } \\
\text { GEOHYD }\end{array}$ & $\begin{array}{l}\text { Used as a } \\
\text { hydrologic } \\
\text { monitoring well. }\end{array}$ & T22S, R31E, S28 & $610 \mathrm{FEL}, 182 \mathrm{FSL}$ & 672,206 & 493,563 & 3465 \\
\hline DOE-2 & $\begin{array}{l}08 / 28 / 84- \\
09 / 18 / 84\end{array}$ & $\begin{array}{l}\text { STRAT, } \\
\text { GEOHYD }\end{array}$ & $\begin{array}{l}\text { Partially plugged/ } \\
\text { conditioned for } \\
\text { deepening. }\end{array}$ & T22S, R31E, S8 & 704 FSL, 128 FEL & 667,317 & 509,876 & 3418 \\
\hline
\end{tabular}


Table 1. DOE-Sponsored Surface Boreholes at the Waste Isolation Pilot Plant (continued)

\begin{tabular}{|c|c|c|c|c|c|}
\hline ID & $\left|\begin{array}{c}\text { Total } \\
\text { Depth }(\mathrm{ft})\end{array}\right|$ & $\begin{array}{l}\text { Depth of } \\
\text { Cored } \\
\text { Interval(s) } \\
\text { (ft) }\end{array}$ & 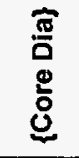 & Reference(s) & Comments \\
\hline \multicolumn{6}{|c|}{ Geologic Evaluation } \\
\hline AEC 7 & $3906^{*}$ & $1028-3906$ & $\left\{4.2^{\prime \prime}\right\}$ & $\begin{array}{l}\text { SAND80-1375 (Christensen and } \\
\text { Peterson, 1981), SAND79-0268 } \\
\text { (Sandia and D'Appolonia, 1983a), } \\
\text { memo: 7/10/74 G.A. Dinwiddie to W.S. } \\
\text { Twenhofel, USGS OFR 81-468 (Jones, } \\
\text { 1981a) }\end{array}$ & $\begin{array}{l}\text { Drilling directed by ORNL. Total depth reached the } \\
\text { lower Castile. Evaluated lithology and stratigraphy of } \\
\text { evaporite sequence for a radioactive waste repository. } \\
\text { Hole completed in repeat section of Anhydrite II. Gas } \\
\text { blowout on } 04 / 19 / 74 \text {. }\end{array}$ \\
\hline $\begin{array}{c}\text { AEC } 7 \\
\text { (deepened) }\end{array}$ & $4720^{*}$ & $3914-4702$ & $\left\{4.2^{\prime \prime}\right\}$ & $\begin{array}{l}\text { SAND79-0268 (Sandia and } \\
\text { D'Appolonia, 1983a), SAND79-0739 } \\
\text { (Christensen, 1979), SAND80-1375 } \\
\text { (Christensen and Peterson, 1981), } \\
\text { SAND89-7056 (Stensrud et al., 1990) }\end{array}$ & $\begin{array}{l}\text { Deepened from the lower Castile to the upper Bell } \\
\text { Canyon to evaluate fluid-bearing zones of Bell } \\
\text { Canyon and to test borehole piugging } \\
\text { operations/materials. Well rehabilitated and casing } \\
\text { perforated in April, } 1988 \text { for hydrologic testing in the } \\
\text { Culebra Dolomite. }\end{array}$ \\
\hline AEC 8 & $3019^{*}$ & $31-3019$ & $\left\{4.2^{\prime \prime}\right\}$ & $\begin{array}{l}\text { SAND79-0269 (Sandia and } \\
\text { D'Appolonia, 1983b), SAND79-0739 } \\
\text { (Christensen, 1979), USGS-WRI 79- } \\
98 \text { (Mercer and Orr, 1979) }\end{array}$ & $\begin{array}{l}\text { Drilling directed by ORNL. Preliminary site } \\
\text { examination. Provided lithologic and stratigraphic } \\
\text { information through the Salado Formation. Hole } \\
\text { completed in Anhydrite III of Castile Formation. }\end{array}$ \\
\hline $\begin{array}{c}\text { AEC } 8 \\
\text { (deepened) }\end{array}$ & $4919^{*}$ & $4809-4863$ & $\left\{4.2^{\prime \prime}\right\}$ & $\begin{array}{l}\text { SAND79-0269 (Sandia and } \\
\text { D'Appolonia, 1983b), SAND79-0739 } \\
\text { (Christensen, 1979), USGS WRI 79- } \\
98 \text { (Mercer and Or, 1979) }\end{array}$ & $\begin{array}{l}\text { Deepened from top of Castile into Ford Shale of Bell } \\
\text { Canyon Formation. Established flow of salt in Castile } \\
\text { halites. Drill stem tests run in Castile Formation show } \\
\text { permeabilities to be in micro-darcies range. }\end{array}$ \\
\hline $\begin{array}{c}\text { B-25 } \\
\text { (BECHTEL 25) }\end{array}$ & 902 & $22-902$ & $\left\{2.2^{\prime \prime}\right\}$ & $\begin{array}{l}\text { Document No. 22-V-510-02 Bechtel } \\
\text { National, Inc. (1979) }\end{array}$ & $\begin{array}{l}\text { Drilled to determine lithology \& stratigraphy for } \\
\text { geologic correlation \& shaft design input, determine } \\
\text { in situ permeability characteristics of selected } \\
\text { horizons, and acquire cores for lab rock testing. } \\
\text { More than } 60 \text { shallow (generally less than } 100^{\prime} \text { ) } \\
\text { holes were drilled for Bechtel to provide information } \\
\text { used in foundation design for surface facilities. } \\
\text { Only B-25 was drilled to significant depth. } \\
\text { Hydrologic testing included the Magenta, the } \\
\text { Culebra, and the Rustler/Salado contact. Hole } \\
\text { completed in upper unit of Salado Formation. }\end{array}$ \\
\hline DOE-1 & $4057^{*}$ & $\begin{array}{l}2135-2376 \\
3342-4057\end{array}$ & $\begin{array}{l}\left\{4.2^{\prime \prime}\right\} \\
\left\{4.2^{\prime \prime}\right\}\end{array}$ & $\begin{array}{l}\text { TME } 3159 \text { (Freeland, 1982), SAND85- } \\
7206 \text { (Hydro Geo Chem, Inc., 1985), } \\
\text { SAND86-7109 (Intera Technologies, } \\
\text { Inc., 1986) }\end{array}$ & $\begin{array}{l}\text { Drilled to acquire stratigraphic data and to } \\
\text { investigate a seismically inferred structural anomaly } \\
\text { (anticlinal) in Castile Formation and test for gas or } \\
\text { fluids associated with anticlinal structures. No } \\
\text { structural disruption in halite \& anhydrite units } \\
\text { observed; no brine or gas found. Hole bottomed in } \\
\text { Anhydrite I of Castile Formation. }\end{array}$ \\
\hline DOE-2 & 981 & $48-981$ & $\left\{2.2^{\prime \prime}\right\}$ & $\begin{array}{l}\text { SAND86-0611 (Mercer et al., 1987), } \\
\text { SAND86-0954 (Mercer, 1987), } \\
\text { SAND86-7166 (Saulnier et al., 1986) }\end{array}$ & $\begin{array}{l}\text { Drilled to investigate a structural depression about } \\
\text { two miles north of WIPP center. Halite is absent from } \\
\text { the Forty-niner \& Tamarisk Members; halite is present } \\
\text { in the lower member of the Rustler Formation. Hole } \\
\text { completed in upper Salado. }\end{array}$ \\
\hline
\end{tabular}


Table 1. DOE-Sponsored Surface Boreholes at the Waste Isolation Pilot Plant (continued)

\begin{tabular}{|c|c|c|c|c|c|c|c|c|}
\hline ID & $\begin{array}{c}\text { Drilling } \\
\text { Start/End } \\
\text { Date }\end{array}$ & Purpose & Status & $\begin{array}{c}\text { Township/ } \\
\text { Rangel } \\
\text { Section }\end{array}$ & $\begin{array}{c}\text { Section } X \& Y \\
\text { Coordinates }\end{array}$ & $\begin{array}{c}\text { State- } \\
\text { Plane } \\
\text { Coord. } \\
X \text { (ft) }\end{array}$ & $\begin{array}{c}\text { State- } \\
\text { Plane } \\
\text { Coord. } \\
\text { Y (ft) }\end{array}$ & $\begin{array}{c}\text { Surface } \\
\text { Elevation } \\
\text { (ft) }\end{array}$ \\
\hline $\begin{array}{c}\text { DOE-2 } \\
\text { (deepened) }\end{array}$ & $\begin{array}{l}04 / 29 / 85- \\
06 / 08 / 85\end{array}$ & $\begin{array}{l}\text { RCHYD, } \\
\text { STRAT }\end{array}$ & $\begin{array}{l}\text { Used for } \\
\text { hydrologic } \\
\text { monitoring of } \\
\text { Rustler Formation } \\
\text { and is isolated by } \\
\text { packer below base } \\
\text { of Rustler. }\end{array}$ & T22S, R31E, S8. & 704 FSL, 128 FEL & 667,317 & 509,876 & 3418 \\
\hline ERDA 6 & $\begin{array}{c}06 / 13 / 75- \\
09 / 18 / 75\end{array}$ & MULTI & $\begin{array}{l}\text { Plugged back } 2773 \\
\text { to } 2560 \mathrm{ft} \text { with } 225 \\
\text { sacks cement. }\end{array}$ & T21S, R31E, S35 & $2152 \mathrm{FSL}, 910 \mathrm{FEL}$ & 682,279 & 521,970 & 3540 \\
\hline ERDA 9 & $\begin{array}{c}04 / 28 / 76 \\
06 / 04 / 76\end{array}$ & MULTI & $\begin{array}{l}\text { Used as a } \\
\text { hydrologic. } \\
\text { monitoring well. }\end{array}$ & T22S, R31E, S20 & 267 FSL, 177 FEL & 667,301 & 498,887 & 3409 \\
\hline ERDA 10 & $\begin{array}{c}08 / 18 / 77 \\
10 / 14 / 77\end{array}$ & $\begin{array}{l}\text { GEO, } \\
\text { BHP }\end{array}$ & $\begin{array}{l}\text { Hole plugged to } \\
\text { surface on } \\
10 / 14 / 77\end{array}$ & T23S, R30E, S34 & $2327 \mathrm{FEL}, 200 \mathrm{FNL}$ & 644,058 & 461,520 & 3371 \\
\hline WPP 11 & $\begin{array}{c}02 / 06 / 78- \\
03 / 14 / 78\end{array}$ & $\begin{array}{l}\text { GEO, } \\
\text { STRAT }\end{array}$ & $\begin{array}{l}\text { Hole plugged and } \\
\text { abandoned by } \\
\text { DOE/Sandia. }\end{array}$ & T22S, R31E, S9 & 294 FWL, 709 FNL & 667,700 & 513,751 & 3426 \\
\hline WIPP 12 & $\begin{array}{l}11 / 09 / 78- \\
12 / 07 / 78\end{array}$ & $\begin{array}{l}\text { GEO, } \\
\text { STRAT }\end{array}$ & $\begin{array}{l}\text { Partially } \\
\text { plugged/standby } \\
\text { condition. }\end{array}$ & T22S, R31E, S17 & $150 \mathrm{FSL}, 80 \mathrm{FEL}$ & 667,371 & 504,068 & 3472 \\
\hline $\begin{array}{l}\text { MIPP } 12 \\
\text { (deepened) }\end{array}$ & $\begin{array}{l}11 / 17 / 81- \\
01 / 01 / 82\end{array}$ & $\begin{array}{c}\text { GEO } \\
\text { GEOHYD }\end{array}$ & $\begin{array}{l}\text { Used as a } \\
\text { hydrologic } \\
\text { monitoring well. } \\
\text { Plugged back to } \\
\text { monitor Culebra. }\end{array}$ & T22S, R31E, S17 & $150 \mathrm{FSL}, 80 \mathrm{FEL}$ & 667,371 & 504,068 & 3472 \\
\hline WPP 13 & $\begin{array}{l}07 / 26 / 78- \\
08 / 06 / 78\end{array}$ & $\begin{array}{l}\text { GEO, } \\
\text { BREC }\end{array}$ & $\begin{array}{l}\text { Used as a } \\
\text { hydrologic } \\
\text { monitoring well. }\end{array}$ & T22S, R31E, S17 & '2567 FSL, $1729 \mathrm{FWL}$ & 663,885 & 506,464 & 3405 \\
\hline $\begin{array}{l}\text { WIPP } 13 \\
\text { (deepened) }\end{array}$ & $\begin{array}{c}08 / 26 / 79- \\
10 / 05 / 79\end{array}$ & $\begin{array}{l}\text { GEO, } \\
\text { STRAT, } \\
\text { GEOHYD }\end{array}$ & $\begin{array}{l}\text { Used as a } \\
\text { hydrologic } \\
\text { monitoring well. }\end{array}$ & T22S, R31E, S17 & 2567 FSL, 1729 FWL & 663,885 & 506,464 & 3405 \\
\hline
\end{tabular}


Table 1. DOE-Sponsored Surface Boreholes at the Waste Isolation Pilot Plant (continued)

\begin{tabular}{|c|c|c|c|c|c|}
\hline ID & $\begin{array}{c}\text { Total } \\
\text { Depth }(f t)\end{array}$ & $\begin{array}{l}\text { Depth of } \\
\text { Cored } \\
\text { Interval(s) } \\
\text { (ft) }\end{array}$ & 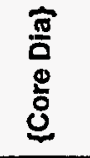 & Reference(s) & Comments \\
\hline $\begin{array}{l}\text { DOE-2 } \\
\text { (deepened) }\end{array}$ & 4325 & $1011-4325$ & $\left\{4.2^{\prime \prime}\right\}$ & $\begin{array}{l}\text { SAND86-0611 (Mercer et al., 1987), } \\
\text { SAND86-0954 (Mercer, 1987) }\end{array}$ & $\begin{array}{l}\text { Hole deepened into the Bell Canyon to investigate } \\
\text { deep dissolution throughout the Castile Formation. } \\
\text { Evidence supporting deep dissolution not observed. } \\
\text { Hole completed in Hays sandstone of Bell Canyon } \\
\text { Formation. }\end{array}$ \\
\hline ERDA 6 & 2775 & $\begin{array}{r}166-171 \\
212-217 \\
499-504 \\
693-695 \\
835-2775\end{array}$ & $\begin{array}{l}\left\{2.0^{\prime \prime}\right\} \\
\left\{2.0^{\prime \prime}\right\} \\
\left\{2.0^{\prime \prime}\right\} \\
\left\{2.0^{\prime \prime}\right\} \\
\left\{2.0^{\prime \prime}\right\}\end{array}$ & $\begin{array}{l}\text { SAND77-0946 (Griswold, 1977a), } \\
\text { SAND79-0267 (Sandia and USGS, } \\
\text { 1983a), USGS OFR 81-468 (Jones, } \\
\text { 1981a), WTSD-TME-3153 (Popielak et } \\
\text { al., 1983) }\end{array}$ & $\begin{array}{l}\text { Drilled to evaluate stratigraphy at initial proposed } \\
\text { WPP site. Established pronounced anticlinal } \\
\text { structure, salt flow, deformation \& fracturing of } \\
\text { Castile anhydrites. At } 2711 \mathrm{ft} \text {, encountered } \\
\text { pressurized brine and dissolved } \mathrm{H}_{2} \mathrm{~S} \text {. After WPP } \\
12 \text { encountered brine, ERDA } 6 \text { was re-entered } \\
\text { (02/19/82 to } 05 / 10 / 83 \text { ) to test its brine reservoir. } \\
\text { The two reservoirs falled to respond to one another. }\end{array}$ \\
\hline ERDA 9 & $2877^{*}$ & $1085-2859$ & $\left\{4.2^{\prime \prime}\right\}$ & $\begin{array}{l}\text { SAND77-0946 (Griswold, 1977a), } \\
\text { SAND80-7119 (Griswold and } \\
\text { McWhiter, 1981), SAND79-0270 } \\
\text { (Sandia and USGS, 1983b), SAND87- } \\
7125 \text { (Stensrud et al., 1987), USGS } \\
\text { OFR 81-469 (Jones, 1981b) }\end{array}$ & $\begin{array}{l}\text { Centered on second proposed WPP site southwest } \\
\text { of ERDA 6. Confirmed satisfactory stratigraphy, } \\
\text { lithology, and mineralogy. Drill stem tests showed } \\
\text { no significant amount of fluid within the Salado. } \\
\text { Hole bottomed } 50 \mathrm{ft} \text { into the Castile Formation in } \\
\text { Anhydrite lil. Hole completed in Anhydrite III of } \\
\text { Castile Formation. }\end{array}$ \\
\hline ERDA 10 & $4417^{*}$ & $2082-4417$ & $\left\{4.2^{21}\right\}$ & $\begin{array}{l}\text { SAND79-0271 (Sandia and } \\
\text { D'Appolonia, 1983C) }\end{array}$ & $\begin{array}{l}\text { Drilled to evaluate dissolution. Hole completed in } \\
\text { Olds sandstone of Bell Canyon Formation. }\end{array}$ \\
\hline WPP 11 & $3570^{\star}$ & $714-3521$ & $\left\{4.2^{m}\right\}$ & $\begin{array}{l}\text { SAND79-0272 (Sandia and USGS, } \\
\text { 1982) }\end{array}$ & $\begin{array}{l}\text { Drilled to evaluate presence/absence of salt } \\
\text { deformation. Confirmed an anticline in the Castile. } \\
\text { No brine encountered. Salt present. Hole completed } \\
\text { in Anhydrite I of Castile Formation. }\end{array}$ \\
\hline WPP 12 & $2776^{*}$ & $\begin{array}{c}123-2759 \\
\text { (discontin- } \\
\text { uous) }\end{array}$ & $\left\{4.2^{\prime \prime}\right\}$ & $\begin{array}{l}\text { SAND82-2336 (Sandia and } \\
\text { D'Appolonia, 1982a) }\end{array}$ & $\begin{array}{l}\text { Drilled to evaluate presence/absence of salt } \\
\text { deformation. Stratigraphy confirmed to top of Castile, } \\
\text { indicated thinning of lower Salado \& more clay searns } \\
\text { exist relative to ERDA 9. Hole completed in upper } \\
\text { unit of Salado Formation. }\end{array}$ \\
\hline $\begin{array}{l}\text { WPP } 12 \\
\text { (deepened) }\end{array}$ & $3928^{*}$ & $2776-3928$ & $\left\{4.2^{\prime \prime}\right\}$ & $\begin{array}{l}\text { TME } 3148 \text { (Black, 1982), SAND86- } \\
7166 \text { (Sauinier et al., 1986), SAND87- } \\
7125 \text { (Stensrud et al., 1987), SAND88- } \\
7014 \text { (Stensrud et al., 1988b) }\end{array}$ & $\begin{array}{l}\text { Deepened to: (1) investigate presence of anticlinal or } \\
\text { domal structure \& thickening of halite indicated by } \\
\text { seismic reflection surveys, (2) determine the nature } \\
\text { and extent of deformation in Castile Formation, and } \\
\text { (3) characterize any fluid-bearing Castile zones. } \\
\text { Encountered pressurized brine in Castile. Hole } \\
\text { completed in Anhydrite l of Castile Formation. }\end{array}$ \\
\hline WPP 13 & $1025^{*}$ & $\begin{array}{l}570-595 \\
656-729 \\
827-878\end{array}$ & $\left\{4.2^{\prime \prime}\right\}$ & $\begin{array}{l}\text { SAND79-0273 (Sandia and USGS, } \\
\text { 1979a), SAND87-7125 (Stensrud et al., } \\
\text { 1987) }\end{array}$ & $\begin{array}{l}\text { Drilled to evaluate resistivity anomaly. No evidence of } \\
\text { dissolution breccia pipe. Hole completed in upper unit } \\
\text { of Salado Formation. }\end{array}$ \\
\hline $\begin{array}{l}\text { WPP } 13 \\
\text { (deepened) }\end{array}$ & $3850^{*}$ & $1023-3850$ & $\left\{4.2^{\prime}\right\}$ & $\begin{array}{l}\text { SAND82-1880 (Sandia and } \\
\text { D'Appolonia, 1982b), SAND87-7125 } \\
\text { (Stensrud et al., 1987), SAND94-0991 } \\
\text { (Powers, 1996) }\end{array}$ & $\begin{array}{l}\text { Deepened to obtain structural information on } \\
\text { deformation in Castile \& lower Salado \& obtain } \\
\text { coreffluid samples to help determine age \& origin of a } \\
\text { presumed disturbed zone. Hole completed in } \\
\text { Anhydrite I of Castile Formation. }\end{array}$ \\
\hline
\end{tabular}


Table 1. DOE-Sponsored Surface Boreholes at the Waste Isolation Pilot Plant (continued)

\begin{tabular}{|c|c|c|c|c|c|c|c|c|}
\hline ID & $\begin{array}{l}\text { Drilling } \\
\text { Start/End } \\
\text { Date }\end{array}$ & Purpose & Status & $\begin{array}{l}\text { Township/ } \\
\text { Rangel } \\
\text { Section }\end{array}$ & $\begin{array}{l}\text { Section } X \& Y \\
\text { Coordinates }\end{array}$ & $\begin{array}{l}\text { State- } \\
\text { Plane } \\
\text { Coord. } \\
X \text { (ft) }\end{array}$ & $\begin{array}{c}\text { State- } \\
\text { Plane } \\
\text { Coord. } \\
Y(\mathrm{ft})\end{array}$ & $\begin{array}{c}\text { Surface } \\
\text { Elevation } \\
\text { (ft) }\end{array}$ \\
\hline WPP 14 & $\begin{array}{c}05 / 01 / 81- \\
06 / 08 / 81\end{array}$ & $\begin{array}{l}\text { GEO, } \\
\text { STRAT }\end{array}$ & $\begin{array}{l}\text { Plugged and } \\
\text { abandoned by } \\
\text { DOE. }\end{array}$ & T22S, R31E, S9 & 97 FSL, 2104 FEL & $670,628^{1}$ & $509,285^{1}$ & 3429 \\
\hline WIPP 15 & $\begin{array}{l}03 / 08 / 78- \\
04 / 05 / 78\end{array}$ & $\begin{array}{l}\text { GEO, } \\
\text { STRAT }\end{array}$ & $\begin{array}{l}\text { Hole loaded with } \\
\text { mud and } \\
\text { temporarily capped } \\
\text { pending further } \\
\text { testing and/or } \\
\text { plugging. Hole } \\
\text { relinquished to land } \\
\text { owner on } 08 / 02 / 78 \\
\text { for use as water } \\
\text { well. }\end{array}$ & T23S, R35E, S18 & $2426 \mathrm{FNL}, 1973 \mathrm{FWL}$ & $\begin{array}{c}785,854 \\
\text { (est) }\end{array}$ & $\begin{array}{c}475,695 \\
\text { (est) }\end{array}$ & 3269 \\
\hline WIPP 16 & $\begin{array}{c}01 / 11 / 80- \\
02 / 08 / 80\end{array}$ & $\begin{array}{l}\text { BREC, } \\
\text { GEO }\end{array}$ & $\begin{array}{l}\text { Plugged and } \\
\text { abandoned by } \\
\text { DOE. }\end{array}$ & T21S, R30E, S5 & 2357 FSL, 139 FWL & 630,443 & 548,609 & 3383 \\
\hline WPP 18 & $\begin{array}{c}03 / 14 / 78- \\
03 / 30 / 78\end{array}$ & $\begin{array}{l}\text { STRAT, } \\
\text { GEO }\end{array}$ & $\begin{array}{l}\text { Cleaned out and } \\
\text { cased hole } \\
10 / 03 / 85 \text { - } \\
10 / 11 / 85 \text {. Hole } \\
\text { currently used as a } \\
\text { Culebra hydroiogic } \\
\text { monitoring well. }\end{array}$ & T22S, R31E, S20 & $984 \mathrm{FNL}, 12 \mathrm{FEL}$ & 667,446 & 502,935 & 3456 \\
\hline WIPP 19 & $\begin{array}{c}04 / 06 / 78- \\
05 / 08 / 78\end{array}$ & $\begin{array}{l}\text { STRAT, } \\
\text { GEO }\end{array}$ & $\begin{array}{l}\text { Cleaned out and } \\
\text { cased hole } \\
09 / 28 / 85 \text { - } \\
10 / 02 / 85 \text {. Hole } \\
\text { currently used as a } \\
\text { Culebra hydrologic } \\
\text { monitoring well. }\end{array}$ & T22S, R31E, S20 & $2287 \mathrm{FNL}, 13 \mathrm{FEL}$ & 667,453 & 501,632 & 3433 \\
\hline WPP 21 & $\begin{array}{l}05 / 24 / 78- \\
05 / 26 / 78\end{array}$ & $\begin{array}{l}\text { STRAT, } \\
\text { GEO }\end{array}$ & $\begin{array}{l}\text { Cleaned out and } \\
\text { cased } 09 / 18 / 85 \text { - } \\
10 / 06 / 85 . \text { Hole } \\
\text { currently used as a } \\
\text { Culebra hydrologic } \\
\text { monitoring well. }\end{array}$ & T22S, R31E, S20 & 1451 FSL, 12 FEL & 667,459 & 500,071 & 3417 \\
\hline WIPP 22 & $\begin{array}{c}05 / 08 / 78- \\
05 / 23 / 78\end{array}$ & $\begin{array}{l}\text { STRAT, } \\
\text { GEO }\end{array}$ & $\begin{array}{l}\text { Cleaned out and } \\
\text { cased hole } \\
09 / 23 / 85- \\
10 / 08 / 85 \text {. Hole } \\
\text { currently used as a } \\
\text { Culebra hydrologic } \\
\text { monitoring well. }\end{array}$ & T22S, R31E, S20 & 2545 FSL, 11 FEL & 667,453 & 501,165 & 3426 \\
\hline
\end{tabular}


Table 1. DOE-Sponsored Surface Boreholes at the Waste Isolation Pilot Plant (continued)

\begin{tabular}{|c|c|c|c|c|c|}
\hline ID & $\begin{array}{c}\text { Total } \\
\text { Depth }(\mathrm{ft})\end{array}$ & $\begin{array}{l}\text { Depth of } \\
\text { Cored } \\
\text { Interval(s) } \\
\text { (ft) }\end{array}$ & $\begin{array}{l}\text { 菑 } \\
\text { @ } \\
\stackrel{0}{0}\end{array}$ & Reference(s) & Comments \\
\hline WIPP 14 & 1000 & $15-957$ & $\{2.2 "\}$ & $\begin{array}{l}\text { SAND82-1783 (Sandia and } \\
\text { D'Appolonia, 1982C) }\end{array}$ & $\begin{array}{l}\text { Drilled in shallow surface depression (karst-like } \\
\text { feature) centered on a negative gravity anomaly. } \\
\text { Rustler Formation is less dense than in adjacent } \\
\text { holes, but believed too deep to account for } \\
\text { observed gravity anomaly. Dewey Lake } \\
\text { stratigraphy nomal. Hole completed in upper unit } \\
\text { of Salado Formation. }\end{array}$ \\
\hline WPP 15 & 811 & $0-810$ & $\left\{2.0^{\prime \prime}\right\}$ & $\begin{array}{l}\text { SAND79-0274 (Sandia and University } \\
\text { of New Mexico, 1981), SAND94-0991 } \\
\text { (Powers, 1996) }\end{array}$ & $\begin{array}{l}\text { Hole drilled to recover material for paleoclimate } \\
\text { evaluation at San Simon sink. State-plane } \\
\text { coordinates are estimated (Gonzales, 1989). Hole } \\
\text { completed in Santa Rosa Formation. }\end{array}$ \\
\hline WIPP 16 & 1300 & $123-1300$ & $\left\{2.2^{\prime \prime}\right\}$ & $\begin{array}{l}\text { USGS OFR 82-968 (Snyder and Gard, } \\
\text { 1982), SAND94-0991 (Powers, 1996) }\end{array}$ & $\begin{array}{l}\text { Drilled to depth of nearby Mississippi Chemical } \\
\text { potash mine to characterize breccia pipe and to } \\
\text { determine stratigraphic displacement of beds. } \\
\text { Confirmed collapse of strata to lower Rustler, } \\
\text { though stratigraphic order is maintained. Halite \& } \\
\text { anhydrite present within cores. Hole completed in } \\
\text { rubble of lower member of Rustler Formation. }\end{array}$ \\
\hline WIPP 18 & 1060 & Not Cored & & $\begin{array}{l}\text { SAND79-0275 (Sandia and USGS, } \\
\text { 1980a), SAND86-7166 (Saulnier et al., } \\
\text { 1986) }\end{array}$ & $\begin{array}{l}\text { Drilled to investigate possible fault in Rustler } \\
\text { Formation or Dewey Lake Red Beds as interpreted } \\
\text { from seismic reflection records. Flat, correlative } \\
\text { beds above Salado Formation were indicated, } \\
\text { contradicting previous seismic reflection data } \\
\text { interpretations. Hole completed in upper member } \\
\text { of Salado Formation. }\end{array}$ \\
\hline WPP 19 & 1038 & $8-1038$ & $\left\{2.0^{\prime \prime}\right\}$ & $\begin{array}{l}\text { SAND79-0276 (Sandia and USGS, } \\
\text { 19806), SAND86-7166 (Saulnier et al., } \\
\text { 1986) }\end{array}$ & $\begin{array}{l}\text { Drilled to investigate possible fault in Rustler } \\
\text { Formation or Dewey Lake Red Beds interpreted } \\
\text { from seismic reflection records. Flat, correlative } \\
\text { beds above Salado Formation were indicated, } \\
\text { contradicting previous seismic reflection data } \\
\text { interpretations. Hole completed in upper member } \\
\text { of Salado Formation. }\end{array}$ \\
\hline WPP 21 & 1045 & Not Cored & & $\begin{array}{l}\text { SAND79-0277 (Sandia and USGS, } \\
\text { 1980C), SAND86-7166 (Saulnier et al., } \\
\text { 1986) }\end{array}$ & $\begin{array}{l}\text { Drilled to investigate possible fault in Rustler } \\
\text { Formation or Dewey Lake Red Beds interpreted } \\
\text { from seismic reflection records. Flat, correlative } \\
\text { beds above Salado Formation were indicated } \\
\text { contradicting previous seismic reflection data } \\
\text { interpretations. Hole completed in upper member of } \\
\text { Salado Formation. }\end{array}$ \\
\hline WIPP 22 & 1450 & Not Cored & & $\begin{array}{l}\text { SAND79-0278 (Sandia and USGS, } \\
\text { 1980d), SAND86-7166 (Saulnier et al., } \\
\text { 1986) }\end{array}$ & $\begin{array}{l}\text { Drilled to investigate possible fault in Rustler } \\
\text { Formation or Dewey Lake Red Beds interpreted } \\
\text { from seismic reflection records. Flat, correlative } \\
\text { beds above Salado Formation were indicated, } \\
\text { contradicting previous seismic reflection data } \\
\text { interpretations. Hole completed in McNutt potash } \\
\text { unit of Salado Formation. }\end{array}$ \\
\hline
\end{tabular}


Table 1. DOE-Sponsored Surface Boreholes at the Waste Isolation Pilot Plant (continued)

\begin{tabular}{|c|c|c|c|c|c|c|c|c|}
\hline ID & $\begin{array}{c}\text { Drilling } \\
\text { Start/End } \\
\text { Date }\end{array}$ & Purpose & Status & $\begin{array}{l}\text { Township/ } \\
\text { Rangel } \\
\text { Section }\end{array}$ & $\begin{array}{c}\text { Section } X \& Y \\
\text { Coordinates }\end{array}$ & $\begin{array}{l}\text { State- } \\
\text { Plane } \\
\text { Coord. } \\
X \text { (ft) }\end{array}$ & $\begin{array}{c}\text { State- } \\
\text { Plane } \\
\text { Coord. } \\
Y(\mathrm{ft})\end{array}$ & $\begin{array}{c}\text { Surface } \\
\text { Elevation } \\
\text { (ft) }\end{array}$ \\
\hline WIPP 25 & $\begin{array}{c}08 / 28 / 78 \\
09 / 07 / 78\end{array}$ & $\begin{array}{l}\text { GEO. } \\
\text { GEOHYD }\end{array}$ & $\begin{array}{l}\text { Used as a } \\
\text { hydrologic } \\
\text { monitoring well. }\end{array}$ & T22S, R30E, S15 & 1853 FSL, 2838 FEL & 643,343 & 505,868 & 3213 \\
\hline WPP 26 & $\begin{array}{l}08 / 28 / 78- \\
09 / 06 / 78\end{array}$ & $\begin{array}{l}\text { GEO, } \\
\text { GEOHYD }\end{array}$ & $\begin{array}{l}\text { Used as a } \\
\text { hydrologic } \\
\text { monitoring well. }\end{array}$ & T22S, R30E, S29 & 2232 FNL, 12 FEL & 635,509 & 496,516 & 3152 \\
\hline WPPP 27 & $\begin{array}{l}09 / 12 / 78 \\
10 / 09 / 78\end{array}$ & $\begin{array}{c}\text { GEO, } \\
\text { GEOHYD }\end{array}$ & $\begin{array}{l}\text { Used as a } \\
\text { hydrologic } \\
\text { monitoring well. }\end{array}$ & T21S, R30E, S21 & $90 \mathrm{FNL}, 1485 \mathrm{FWL}$ & 637,103 & 535,612 & 3177 \\
\hline WPP 28 & $\begin{array}{c}08 / 07 / 78- \\
08 / 25 / 78\end{array}$ & $\begin{array}{l}\text { GEO, } \\
\text { GEOHYD }\end{array}$ & $\begin{array}{l}\text { Used as a } \\
\text { hydrologic } \\
\text { monitoring well. }\end{array}$ & T21S, R31E, S18 & 99 FNL, 2401 FEL & 659,579 & 540,722 & 3347 \\
\hline WIPP 29 & $\begin{array}{l}10 / 02 / 78- \\
10 / 10 / 78\end{array}$ & $\begin{array}{c}\text { GEO, } \\
\text { GEOHYD }\end{array}$ & $\begin{array}{l}\text { Used as a } \\
\text { hydrologic } \\
\text { monitoring well. }\end{array}$ & T22S, R29E, S34 & $407 \mathrm{FSL}, 1828 \mathrm{FEL}$ & 612,378 & 488,559 & 2977 \\
\hline WIPP 30 & $\begin{array}{c}09 / 08 / 78- \\
09 / 23 / 78\end{array}$ & $\begin{array}{c}\text { GEO, } \\
\text { GEOHYD }\end{array}$ & $\begin{array}{l}\text { Used as a } \\
\text { hydrologic } \\
\text { monitoring well. }\end{array}$ & T21S, R31E, S33 & $668 \mathrm{FNL}, 177 \mathrm{FWL}$ & 667,536 & 524,337 & 3428 \\
\hline WAPP 31 & $\begin{array}{c}09 / 18 / 78- \\
10 / 04 / 78\end{array}$ & $\begin{array}{l}\text { GEO, } \\
\text { BREC }\end{array}$ & $\begin{array}{l}\text { Used as a } \\
\text { monitoring well for } \\
\text { two years. Plugged } \\
\text { after deepened and } \\
\text { tested. }\end{array}$ & T20S, R30E, S35 & $423 \mathrm{FSL}, 1762 \mathrm{FWL}$ & $619,615^{2}$ & 554,620 & 3401 \\
\hline $\begin{array}{l}\text { WPP } 31 \\
\text { (deepened) }\end{array}$ & $\begin{array}{c}07 / 18 / 80- \\
09 / 29 / 80\end{array}$ & BREC & $\begin{array}{l}\text { Plugged and } \\
\text { abandoned. }\end{array}$ & T20S, R30E, S35 & $423 \mathrm{FSL}, 1762 \mathrm{FW}$ & $619,615^{2}$ & 554,620 & 3401 \\
\hline WPP 32 & $\begin{array}{c}08 / 07 / 79- \\
08 / 16 / 79\end{array}$ & $\begin{array}{l}\text { STRAT, } \\
\text { GEO, } \\
\text { BREC }\end{array}$ & $\begin{array}{l}\text { Abandoned on } \\
08 / 22 / 79 \text {. Hole } \\
\text { plugged to surface } \\
\text { with } 500 \mathrm{ft}^{3} \text { of } \\
\text { cement. }\end{array}$ & T22S, R29E, S33 & 1673 FSL, 29 FEL & 608,848 & 489,850 & 3023 \\
\hline WMPP 33 & $\begin{array}{l}07 / 17 / 79- \\
07 / 25 / 79\end{array}$ & $\begin{array}{l}\text { STRAT, } \\
\text { GEO, } \\
\text { BREC }\end{array}$ & $\begin{array}{l}\text { Plugged and } \\
\text { abandoned on } \\
09 / 04 / 79 \text {. }\end{array}$ & T22S, R30E, S13 & $1762 \mathrm{FSL}, 2427 \mathrm{FWL}$ & 653,981 & 505,790 & 3323 \\
\hline WIPP 34 & $\begin{array}{c}08 / 16 / 79- \\
09 / 01 / 79\end{array}$ & GEO & $\begin{array}{l}\text { Plugged and } \\
\text { abandoned on } \\
09 / 04 / 79 \text {. }\end{array}$ & T22S, R31E, $s 9^{3}$ & 202 FSL, 2000 FWL & 669,449 & 509,375 & 3433 \\
\hline
\end{tabular}


Table 1. DOE-Sponsored Surface Boreholes at the Waste Isolation Pilot Plant (continued)

\begin{tabular}{|c|c|c|c|c|c|}
\hline ID & $\begin{array}{c}\text { Total } \\
\text { Depth (ft) }\end{array}$ & $\begin{array}{l}\text { Depth of } \\
\text { Cored } \\
\text { Interval(s) } \\
\text { (ft) }\end{array}$ & 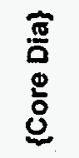 & Reference(s) & Comments \\
\hline WPP 25 & 655 & $\begin{array}{c}0-21 \\
21-238 \\
238-650\end{array}$ & $\begin{array}{l}\left\{2.9^{\prime \prime}\right\} \\
\left\{2.4^{\prime \prime}\right\} \\
\left\{4.0^{\prime \prime}\right\}\end{array}$ & $\begin{array}{l}\text { SAND79-0279 (Sandia and USGS, } \\
\text { 1979b) }\end{array}$ & $\begin{array}{l}\text { Drilled to help define stratigraphy \& hydraulics of } \\
\text { Nash Draw, especially dissolution status. Hole } \\
\text { completed in upper member of Saladio Formation. }\end{array}$ \\
\hline WPP 26 & 503 & $\begin{array}{c}0-10 \\
10-222 \\
222-503\end{array}$ & $\begin{array}{l}\left\{2.9^{\prime \prime}\right\} \\
\left\{2.1^{\prime \prime}\right\} \\
\left\{3.5^{\prime \prime}\right\}\end{array}$ & $\begin{array}{l}\text { SAND79-0280 (Sandia and USGS, } \\
1979 C \text { ) }\end{array}$ & $\begin{array}{l}\text { Drilled to help define stratigraphy \& hydraulics of } \\
\text { Nash Draw, especially dissolution status. Hole } \\
\text { completed in upper member of Salado Formation. }\end{array}$ \\
\hline WPPP 27 & 592 & $\begin{array}{c}0-21 \\
21-592\end{array}$ & $\begin{array}{l}\left\{2.9^{\prime \prime}\right\} \\
\left\{4.0^{\prime \prime}\right\}\end{array}$ & $\begin{array}{l}\text { SAND79-0281 (Sandia and USGS, } \\
\text { 1979d) }\end{array}$ & $\begin{array}{l}\text { Drilled to help define stratigraphy } \& \text { hydraulics of } \\
\text { Nash Draw, especially dissolution status. Hole } \\
\text { completed in upper member of Salado Formation. }\end{array}$ \\
\hline WIPP 28 & 801 & $\begin{array}{c}0-10 \\
10-21 \\
21-801\end{array}$ & $\begin{array}{l}\left\{2.9^{\prime \prime}\right\} \\
\left\{2.3^{\prime \prime}\right\} \\
\left\{3.5^{\prime \prime}\right\}\end{array}$ & $\begin{array}{l}\text { SAND79-0282 (Sandia and USGS, } \\
1979 e \text { ) }\end{array}$ & $\begin{array}{l}\text { Drilled to help define stratigraphy \& hydraulics of } \\
\text { Nash Draw, especially dissolution status. Hole } \\
\text { completed in upper member of Salado Formation. }\end{array}$ \\
\hline WIPP 29 & 378 & $\begin{array}{c}0-5 \\
5-12 \\
12-378\end{array}$ & $\begin{array}{l}\left\{2.9^{11}\right\} \\
\left\{2.1^{11}\right. \\
\left\{2.2^{14}\right\}\end{array}$ & $\begin{array}{l}\text { SAND79-0283 (Sandia and USGS, } \\
\text { 1979f) }\end{array}$ & $\begin{array}{l}\text { Drilled to help define stratigraphy } \& \text { hydraulics of } \\
\text { Nash Draw, especially dissolution status. Hole } \\
\text { completed in MCNutt potash unit of Salado Formation. }\end{array}$ \\
\hline WIPP 30 & 912 & $\begin{array}{c}0-7 \\
7-17 \\
17-912\end{array}$ & $\begin{array}{l}\left\{2.9^{\prime \prime}\right\} \\
\left\{2.0^{\circ \prime}\right\} \\
\left\{3.5^{\prime \prime}\right\}\end{array}$ & $\begin{array}{l}\text { SAND79-0284 (Sandia and USGS, } \\
\text { 1980e), SAND88-7014 (Stensrud et } \\
\text { al.,1988b) }\end{array}$ & $\begin{array}{l}\text { Drilled to define changes in stratigraphy \& hydrology } \\
\text { between WPP site and Nash Draw, especially } \\
\text { dissolution status. Hole completed in upper member } \\
\text { of Salado Formation. }\end{array}$ \\
\hline WIPP 31 & 810 & 459-810 & $\left\{3.5^{\prime \prime}\right\}$ & WPO2732 (Statler, 1978) & $\begin{array}{l}\text { Drilled to characterize breccia pipe. Intermittent } \\
\text { coring to } 810 \mathrm{ft} \text { in initial exploratory hole confirmed } \\
\text { breccia to depths below top of halite in adjacent beds. } \\
\text { Hole completed in a breccia material. }\end{array}$ \\
\hline $\begin{array}{l}\text { MPP } 31 \\
\text { (deepened) }\end{array}$ & 1982 & $819-1982$ & $\left\{3.5^{\prime \prime}\right\}$ & $\begin{array}{l}\text { USGS OFR-82-968 (Snyder and } \\
\text { Gard, 1982), WPO7885 (Powers, } \\
\text { 1980), SAND94-0991 (Powers, 1996) }\end{array}$ & $\begin{array}{l}\text { Cored continuoushy from } 810 \mathrm{ft} \text { to borehole total } \\
\text { depth. Characterized breccia to depths below level of } \\
\text { Yates Formation. Hole completed in breccia material. }\end{array}$ \\
\hline WIPP 32 & 390 & $\begin{array}{c}4-13 \\
13-353\end{array}$ & $\begin{array}{l}\left\{1.5^{\prime \prime}\right\} \\
\left\{2.4^{4}\right\}\end{array}$ & $\begin{array}{l}\text { SAND80-1102 (Sandia and } \\
\text { USGS,1980f), WPO7885 (Powers, } \\
\text { 1980), SAND94-0991 (Powers, 1996) }\end{array}$ & $\begin{array}{l}\text { Drilled on local topographic high point in Nash Draw } \\
\text { considered by some investigators as breccia pipe. } \\
\text { Borehole characterized stratigraphy in upper Salado } \\
\text { to TD. No evidence of breccia pipe. Hole } \\
\text { completed in McNutt potash unit of Salado } \\
\text { Formation. }\end{array}$ \\
\hline WIPP 33 & 840 & $\begin{array}{c}48-58 \\
384-480 \\
540-588 \\
650-700\end{array}$ & $\begin{array}{l}\left\{3.5^{\prime \prime}\right\} \\
\left\{3.5^{\prime \prime}\right\} \\
\left\{3.5^{\prime \prime}\right\} \\
\left\{3.5^{\prime \prime}\right\}\end{array}$ & $\begin{array}{l}\text { SAND80-2011 (Sandia and } \\
\text { USGS,1981a), SAND94-0991 } \\
\text { (Powers, 1996) }\end{array}$ & $\begin{array}{l}\text { Drilled to investigate the stratigraphy and structure of } \\
\text { a small closed depression in the northwestern WIPP } \\
\text { site area. }\end{array}$ \\
\hline WIPP 34 & 1820 & $\begin{array}{l}627-654 \\
654-1793\end{array}$ & $\begin{array}{l}\left\{3.5^{\prime \prime}\right\} \\
\left\{2.4^{4}\right\}\end{array}$ & $\begin{array}{l}\text { SAND81-2643 (Sandia and } \\
\text { USGS,1981b) }\end{array}$ & $\begin{array}{l}\text { Drilled to investigate slight syncline, interpreted as } \\
\text { karst feature, in upper Salado/lower Rustler in } \\
\text { disturbed zone. Drilling data indicated slight } \\
\text { syncline; normal stratigraphy through McNutt } \\
\text { potash zone. Hole completed in lower unit of } \\
\text { Salado Formation. }\end{array}$ \\
\hline
\end{tabular}


Table 1. DOE-Sponsored Surface Boreholes at the Waste Isolation Pilot Plant (continued)

\begin{tabular}{|c|c|c|c|c|c|c|c|c|}
\hline ID & $\begin{array}{c}\text { Drilling } \\
\text { Start/End } \\
\text { Date }\end{array}$ & Purpose & Status & $\begin{array}{c}\text { Township/ } \\
\text { Range/ } \\
\text { Section }\end{array}$ & $\begin{array}{c}\text { Section } X \& Y \\
\text { Coordinates }\end{array}$ & $\begin{array}{l}\text { State- } \\
\text { Plane } \\
\text { Coord. } \\
X \text { (ft) }\end{array}$ & $\begin{array}{c}\text { State- } \\
\text { Plane } \\
\text { Coord. } \\
Y \text { (ft) }\end{array}$ & $\begin{array}{c}\text { Surface } \\
\text { Elevation } \\
\text { (ft) }\end{array}$ \\
\hline \multicolumn{9}{|c|}{ Hydrologic Testing and Monitoring } \\
\hline$H-1$ & $\begin{array}{l}05 / 20 / 76- \\
06 / 09 / 76\end{array}$ & HYD & $\begin{array}{l}\text { Used as a } \\
\text { hydrologic } \\
\text { monitoring well. }\end{array}$ & T22S, R31E, S29 & 1083 FEL, 623 FNL & 666,400 & 497,991 & 3403 \\
\hline $\mathrm{H}-2 \mathrm{a}$ & $\begin{array}{l}02 / 14 / 77 \\
02 / 19 / 77\end{array}$ & HYD & $\begin{array}{l}\text { Used as a } \\
\text { hydrologic } \\
\text { monitoring well. }\end{array}$ & T22S, R31E, S29 & 727 FNL, 1698 FWL & 663,897 & 497,912 & 3377 \\
\hline $\begin{array}{c}\mathrm{H}-2 \mathrm{a} \\
\text { (deepened) }\end{array}$ & $\begin{array}{l}07 / 12 / 83- \\
07 / 17 / 83\end{array}$ & HYD & $\begin{array}{l}\text { Used as a } \\
\text { hydrologic } \\
\text { monitoring well. }\end{array}$ & T22S, R31E, S29 & 727 FNL, $1698 \mathrm{FWL}$ & 663,897 & 497,912 & 3377 \\
\hline $\mathrm{H}-2 \mathrm{~b} 1$ & $\begin{array}{l}02 / 07 / 77- \\
02 / 14 / 77\end{array}$ & HYD & $\begin{array}{l}\text { Used as a } \\
\text { hydrologic } \\
\text { monitoring well. }\end{array}$ & T22S, R31E, S29 & $696 \mathrm{FNL}, 1661 \mathrm{FWL}$ & 663,860 & 497,943 & 3378 \\
\hline $\mathrm{H}-2 \mathrm{~b} 2$ & $\begin{array}{c}07 / 16 / 83- \\
07 / 30 / 83\end{array}$ & HYD & $\begin{array}{l}\text { Used as a } \\
\text { hydrologic } \\
\text { monitoring well. }\end{array}$ & T22S, R31E, S29 & 701 FNL, $1691 \mathrm{FWL}$ & 663,890 & 497,938 & 3377 \\
\hline $\mathrm{H}-2 \mathrm{C}$ & $\begin{array}{l}01 / 28 / 77- \\
02 / 05 / 77\end{array}$ & HYD & $\begin{array}{l}\text { Used as a } \\
\text { hydrologic } \\
\text { monitoring well. }\end{array}$ & T22S, R31E, S29 & $637 \mathrm{FNL}, 1709 \mathrm{FWL}$ & 663,907 & 498,002 & 3377 \\
\hline $\mathrm{H}-3 \mathrm{~b} 1$ & $\begin{array}{l}07 / 25 / 76- \\
08 / 12 / 76\end{array}$ & HYD & $\begin{array}{l}\text { Well used for } \\
\text { hydrologic } \\
\text { monitoring and } \\
\text { tracer testing. }\end{array}$ & T22S, R31E, S29 & 2085 FSL, 138 FEL & 667,377 & 495,440 & 3389 \\
\hline
\end{tabular}


Table 1. DOE-Sponsored Surface Boreholes at the Waste Isolation Pilot Plant (continued)

\begin{tabular}{|c|c|c|c|c|c|}
\hline ID & $\begin{array}{c}\text { Total } \\
\text { Depth }(\mathrm{ft})\end{array}$ & $\begin{array}{l}\text { Depth of } \\
\text { Cored } \\
\text { Interval(s) } \\
\text { (ft) }\end{array}$ & 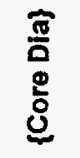 & Reference(s) & Comments \\
\hline \multicolumn{6}{|c|}{ Hydrologic Testing and Monitoring } \\
\hline$H-1$ & $848^{*}$ & $731-842$ & $\left\{4.2^{\prime \prime}\right\}$ & $\begin{array}{l}\text { USGS-WRI 79-98 (Mercer and Orr, } \\
\text { 1979), SAND87-7166 (Stensrud et } \\
\text { al.,1988a) }\end{array}$ & $\begin{array}{l}\text { Drilled for hydraulic testing \& water level monitoring } \\
\text { across the Magenta \& Culebra Dolomites \& the } \\
\text { Rustler/Salado contact. Hole completed in upper } \\
\text { member of Salado Formation to fully expose all three } \\
\text { test intervals. }\end{array}$ \\
\hline $\mathrm{H}-2 \mathrm{a}$ & 563 & $513-563$ & $\left\{2.2^{\prime \prime}\right\}$ & $\begin{array}{l}\text { SAND85-7206 (Hydro Geo Chem, Inc., } \\
\text { 1985), SAND85-7263 (Intera } \\
\text { Technologies, Inc., and Hydro Geo } \\
\text { Chem, Inc., 1985), SAND86-7166 } \\
\text { (Saulnier et al., 1986), SAND87-7166 } \\
\text { (Stensrud et al., 1988a), USGS-WRI } \\
\text { 79-98 (Mercer and Orr, 1979) }\end{array}$ & $\begin{array}{l}\text { Hole was initially completed in the Magenta Dolomite. } \\
\text { Hole later deepened to Tamarisk member of Rustler } \\
\text { Formation to expose a full section of the Magenta. }\end{array}$ \\
\hline $\begin{array}{c}\mathrm{H}-2 \mathrm{a} \\
\text { (deepened) }\end{array}$ & 672 & $616-672$ & $\left\{2.2^{m}\right\}$ & $\begin{array}{l}\text { SAND85-7206 (Hydro Geo Chem, Inc., } \\
\text { 1985), SAND85-7263 (Intera } \\
\text { Technologies, Inc., and Hydro Geo } \\
\text { Chem, Inc., 1985), SAND86-7166 } \\
\text { (Sauinier et al., 1986), SAND87-7166 } \\
\text { (Stensrud et al., 1988a) }\end{array}$ & $\begin{array}{l}\text { Drilled to provide oriented core for subsequent } \\
\text { fracture orientation studies and for } \\
\text { anhydrite/gypsum alteration studies. Other objective } \\
\text { for this hole was to provide test intervals for } \\
\text { groundwater studies. In April } 1984 \text { well was re- } \\
\text { entered because it had degraded. Casing and } \\
\text { screen assembly were installed to top of Culebra. } \\
\text { Hole completed in lower member of Rustler } \\
\text { Formation to expose a full section of the Culebra. }\end{array}$ \\
\hline $\mathrm{H}-2 \mathrm{~b} 1$ & 661 & $611-661$ & $\left\{2.2^{\prime \prime}\right\}$ & $\begin{array}{l}\text { SAND85-7263 (Intera Technologies, } \\
\text { Inc., and Hydro Geo Chem, Inc., 1985), } \\
\text { SAND86-7166 (Sauinier et al., 1986), } \\
\text { SAND87-7166 (Stensrud et al., 1988a), } \\
\text { SAND89-7056 (Stensrud et al., 1990) }\end{array}$ & $\begin{array}{l}\text { Casing perforated in Magenta \& Culebra Dolomites. } \\
\text { Hole later completed in lower member of Rustier } \\
\text { Formation. }\end{array}$ \\
\hline $\mathrm{H}-2 \mathrm{~b} 2$ & 660 & $450-660$ & $\left\{2.2^{\prime \prime}\right\}$ & $\begin{array}{l}\text { SAND85-7206 (Hydro Geo Chem, Inc., } \\
\text { 1985), SAND85-7263 (Intera } \\
\text { Technologies, Inc., and Hydro Geo } \\
\text { Chem, Inc., 1985), SAND86-7166 } \\
\text { (Saulnier et al., 1986), SAND87-7166 } \\
\text { (Stensrud et al., 1988a) }\end{array}$ & $\begin{array}{l}\text { Drilled for monitoring \& testing groundwater in the } \\
\text { Culebra Dolomite. Pumping and slug tests were } \\
\text { conducted to characterize the hydraulic properties } \\
\text { of the Culebra Dolomite \& establish its hydraulic } \\
\text { relationship with overtying/underlying water-bearing } \\
\text { units. Hole completed in lower member of Rustler } \\
\text { Formation. }\end{array}$ \\
\hline $\mathrm{H}-2 \mathrm{c}$ & 795 & $743-795$ & $\left\{2.2^{m}\right\}$ & $\begin{array}{l}\text { SAND85-7263 (Intera Technologies, } \\
\text { Inc., and Hydro Geo Chem, Inc., 1985), } \\
\text { SAND86-7109 (Intera Technologies, } \\
\text { Inc., 1986), SAND86-7 166 (Saulnier et } \\
\text { al., 1986), SAND87-7166 (Stensrud et } \\
\text { al., 1988a), USGS WRI 79-98 (Mercer } \\
\text { and Orr, 1979) }\end{array}$ & $\begin{array}{l}\text { Hole is completed below the Rustler/Salado contact \& } \\
\text { perforated in the Culebra Dolomite. }\end{array}$ \\
\hline $\mathrm{H}-3 \mathrm{~b} 1$ & 902 & $\begin{array}{l}\text { Not } \\
\text { Cored }\end{array}$ & & $\begin{array}{l}\text { SAND85-7206 (Hydro Geo Chem, Inc., } \\
\text { 1985), SAND86-7109 (Intera } \\
\text { Technologies, Inc., 1986), SAND86- } \\
7166 \text { (Sauinier et al., 1986), SAND86- } \\
7177 \text { (Pearson et al., 1987), SAND89- } \\
7056 \text { (Stensrud et al., 1990) }\end{array}$ & $\begin{array}{l}\text { Multi-completion hole where Magenta and Culebra } \\
\text { Dolomite and Rustler/Salado contact were tested. } \\
\text { Hole completed in upper member of Salado } \\
\text { Formation. }\end{array}$ \\
\hline
\end{tabular}


Table 1. DOE-Sponsored Surface Boreholes at the Waste Isolation Pilot Plant (continued)

\begin{tabular}{|c|c|c|c|c|c|c|c|c|}
\hline ID & $\begin{array}{c}\text { Drilling } \\
\text { Start/End } \\
\text { Date }\end{array}$ & Purpose & Status & $\begin{array}{l}\text { Township/ } \\
\text { Rangel } \\
\text { Section }\end{array}$ & $\begin{array}{c}\text { Section X \& Y } \\
\text { Coordinates }\end{array}$ & $\begin{array}{l}\text { State- } \\
\text { Plane } \\
\text { Coord. } \\
\mathrm{X} \text { (ft) }\end{array}$ & $\begin{array}{c}\text { State- } \\
\text { Plane } \\
\text { Coord. } \\
Y(\mathrm{ft})\end{array}$ & $\begin{array}{c}\text { Surface } \\
\text { Elevation } \\
\text { (ft) }\end{array}$ \\
\hline $\mathrm{H}-3 \mathrm{~b} 2$ & $\begin{array}{l}10 / 25 / 83- \\
11 / 08 / 83\end{array}$ & HYD & $\begin{array}{l}\text { Well used for } \\
\text { hydrologic } \\
\text { monitoring and } \\
\text { tracer testing. }\end{array}$ & T22S, R31E, S29 & 2122 FSL, 231 FEL & 667,283 & 495,476 & 3389 \\
\hline$H-3 b 3$ & $\begin{array}{l}11 / 15 / 83- \\
12 / 16 / 83\end{array}$ & HYD & $\begin{array}{l}\text { Well used for } \\
\text { hydrologic } \\
\text { monitoring and } \\
\text { tracer testing. }\end{array}$ & T22S, R31E, S29 & $2022 \mathrm{FSL}, 217 \mathrm{FEL}$ & 667,298 & 495,376 & 3388 \\
\hline $\mathrm{H}-3 \mathrm{~d}$ & $\begin{array}{c}03 / 31 / 87- \\
04 / 22 / 87\end{array}$ & HYD & $\begin{array}{l}\text { Well used for } \\
\text { hydrologic } \\
\text { monitoring and } \\
\text { tracer testing. }\end{array}$ & T22S, R31E, S29 & $2067 \mathrm{FSL}, 164 \mathrm{FEL}$ & 667,350 & 495,421 & 3387 \\
\hline $\mathrm{H}-4 \mathrm{a}$ & $\begin{array}{l}04 / 30 / 78- \\
05 / 2278\end{array}$ & HYD & $\begin{array}{l}\text { Well used for } \\
\text { hydrologic } \\
\text { monitoring and } \\
\text { tracer testing. }\end{array}$ & T23S, R31E, S5 & $546 \mathrm{FNL}, 720 \mathrm{FWL}$ & 662,993 & 486,962 & 3333 \\
\hline$H-4 b$ & $\begin{array}{c}04 / 30 / 78- \\
05 / 15 / 78\end{array}$ & HYD & $\begin{array}{l}\text { Well used for } \\
\text { hydrologic } \\
\text { monitoring and } \\
\text { tracer testing. }\end{array}$ & T23S, R31E, S5 & 498 FNL, 633 FWL & 662,906 & 487,554 & 3333 \\
\hline $\mathrm{H}-4 \mathrm{c}$ & $\begin{array}{c}04 / 30 / 78- \\
05 / 09 / 78\end{array}$ & HYD & $\begin{array}{l}\text { Well used for } \\
\text { hydrologic } \\
\text { monitoring and } \\
\text { tracer testing. }\end{array}$ & T23S, R31E, S5 & $446 \mathrm{FNL}, 718 \mathrm{FWL}$ & 662,991 & 487,607 & 3334 \\
\hline $\mathrm{H}-5 \mathrm{a}$ & $\begin{array}{l}05 / 22 / 78- \\
06 / 20 / 78\end{array}$ & HYD & $\begin{array}{l}\text { Used as a } \\
\text { hydrologic } \\
\text { monitoring well. }\end{array}$ & T22S, R31E, S15 & $1092 \mathrm{FNL}, 185 \mathrm{FEL}$ & 677,828 & 508,111 & 3506 \\
\hline$H-5 b$ & $\begin{array}{l}05 / 22 / 78- \\
06 / 13 / 78\end{array}$ & HYD & $\begin{array}{l}\text { Used as a } \\
\text { hydrologic } \\
\text { monitoring well. }\end{array}$ & T22S, R31E, S15 & 1008 FNL, $236 \mathrm{FEL}$ & 677,777 & 508,194 & 3506 \\
\hline
\end{tabular}


Table 1. DOE-Sponsored Surface Boreholes at the Waste Isolation Pilot Plant (continued)

\begin{tabular}{|c|c|c|c|c|c|}
\hline ID & $\begin{array}{c}\text { Total } \\
\text { Depth }(\mathrm{ft})\end{array}$ & $\begin{array}{l}\text { Depth of } \\
\text { Cored } \\
\text { Interval(s) } \\
\text { (ft) }\end{array}$ & 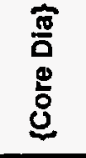 & Reference(s) & Comments \\
\hline $\mathrm{H}-3 \mathrm{~b} 2$ & 725 & $32-725$ & $\left\{2.2^{\prime \prime}\right\}$ & $\begin{array}{l}\text { SAND85-7206 (Hydro Geo Chem, Inc., } \\
\text { 1985), SAND86-7109 (Intera } \\
\text { Technologies, Inc., 1986), SAND86- } \\
7166 \text { (Saulnier et al., 1986), SAND86- } \\
7177 \text { (Pearson et al., 1987), SAND89- } \\
7056 \text { (Stensrud et al., 1990), Appendix } \\
\text { A4; Technical Memorandum (Mercer, } \\
\text { 1994) }\end{array}$ & $\begin{array}{l}\text { Hole is completed in Culebra Dolomite for tracer and } \\
\text { cross hole testing. }\end{array}$ \\
\hline $\mathrm{H}-3 \mathrm{~b} 3$ & 730 & $34-730$ & $\left\{2.2^{n}\right\}$ & $\begin{array}{l}\text { SAND85-7206 (Hydro Geo Chem, Inc., } \\
\text { 1985), SAND86-7109 (Intera } \\
\text { Technologies, Inc., 1986), SAND86- } \\
7166 \text { (Saulnier et al., 1986), SAND86- } \\
7177 \text { (Pearson et al., 1987), Appendix } \\
\text { A }^{4} \text { Technical Memorandum (Mercer, } \\
\text { 1994) }\end{array}$ & $\begin{array}{l}\text { Hole is completed in Culebra Dolomite for tracer and } \\
\text { cross hole testing. }\end{array}$ \\
\hline$H-3 d$ & 554 & $\begin{array}{l}\text { Not } \\
\text { Cored }\end{array}$ & & $\begin{array}{l}\text { SAND85-7206 (Hydro Geo Chem, } \\
\text { Inc., 1985), SAND86-7109 (Intera } \\
\text { Technologies, Inc., 1986), SAND86- } \\
7166 \text { (Sauinier et al., 1986), SAND86- } \\
7177 \text { (Pearson et al., 1987), Sanchez } \\
\text { and McCasland (1994), SAND89- } \\
\text { 7056 (Stensrud et al., 1990), Appendix } \\
\text { A.; Technical Memorandum (Mercer, }^{4} \\
\text { 1994) }\end{array}$ & $\begin{array}{l}\text { Hole is open from bottom of surface casing at } 39 \mathrm{ft} \\
\text { Hole completed to test Dewy Lake Formation and is } \\
\text { used as a water level observation well. This borehole } \\
\text { originally was called H-3b4. }\end{array}$ \\
\hline $\mathrm{H}-4 \mathrm{a}$ & 532 & $365-415$ & $\left\{2.2^{\prime \prime}\right\}$ & $\begin{array}{l}\text { SAND85-7206 (Hydro Geo Chem, Inc., } \\
\text { 1985), SAND86-7166 (Saulnier et al., } \\
\text { 1986), USGS-WRI } 81-36 \text { (Mercer et } \\
\text { al., 1981) }\end{array}$ & $\begin{array}{l}\text { Hole originally completed in the Magenta Dolomite, } \\
\text { later deepened to the lower member of Rustler } \\
\text { Formation. }\end{array}$ \\
\hline $\mathrm{H}-4 \mathrm{~b}$ & 529 & $477-529$ & $\left\{2.2^{\prime \prime}\right\}$ & $\begin{array}{l}\text { USGS-WRI 81-36 (Mercer et al., } \\
\text { 1981), SAND85-7206 (Hydro Geo } \\
\text { Chem, Inc., 1985), SAND86-7109 } \\
\text { (Intera Technologies, Inc., 1986), } \\
\text { SAND86-7166 (Saulnier et al., 1986) }\end{array}$ & $\begin{array}{l}\text { Completed in Culebra Dolomite. Hole bottomed in } \\
\text { lower member of Rustler Formation. }\end{array}$ \\
\hline$H-4 c$ & 661 & $611-661$ & $\left\{2.2^{\prime \prime}\right\}$ & $\begin{array}{l}\text { SAND85-7206 (Hydro Geo Chem, Inc., } \\
\text { 1985), SAND86-7166 (Saulnier et al., } \\
\text { 1986), SAND87-7125 (Stensrud et al., } \\
\text { 1987), USGS-WRI 81-36 (Mercer et } \\
\text { al., 1981), USGS WRI 79-98 (Mercer } \\
\text { and Orr, 1979) }\end{array}$ & $\begin{array}{l}\text { Completed in the Rustler/Salado contact \& the } \\
\text { Culebra Dolomite. Hole bottomed in upper member } \\
\text { of Salado Formation. }\end{array}$ \\
\hline $\mathrm{H}-5 \mathrm{a}$ & 930 & $775-824$ & $\left\{2.2^{11}\right\}$ & $\begin{array}{l}\text { USGS-WRI 82-19 (Dennehy and } \\
\text { Mercer, 1982), SAND82-0080 } \\
\text { (Seward, 1982), SAND85-7206 (Hydro } \\
\text { Geo Chem, Inc., 1985), SAND86-7166 } \\
\text { (Sauinier et al., 1986) }\end{array}$ & $\begin{array}{l}\text { Hole originally completed in the Magenta Dolomite, } \\
\text { later deepened to the lower member of Rustler } \\
\text { Formation. }\end{array}$ \\
\hline$H-5 b$ & 925 & $882-925$ & $\left\{2.2^{\prime \prime}\right\}$ & $\begin{array}{l}\text { USGS-WRI 82-19 (Dennehy and } \\
\text { Mercer, 1982), SAND82-0080 } \\
\text { (Seward, 1982), SAND85-7206 (Hydro } \\
\text { Geo Chem, Inc., 1985), SAND86-7166 } \\
\text { (Saulnier et al., 1986) }\end{array}$ & $\begin{array}{l}\text { Hole is completed in the Culebra Dolomite. Hole } \\
\text { bottomed in lower member of Rustler Formation. }\end{array}$ \\
\hline
\end{tabular}


Table 1. DOE-Sponsored Surface Boreholes at the Waste Isolation Pilot Plant (continued)

\begin{tabular}{|c|c|c|c|c|c|c|c|c|}
\hline ID & $\begin{array}{l}\text { Drilling } \\
\text { Start/End } \\
\text { Date }\end{array}$ & Purpose & Status & $\begin{array}{l}\text { Township/ } \\
\text { Range/ } \\
\text { Section }\end{array}$ & $\begin{array}{l}\text { Section } X \& Y \\
\text { Coordinates }\end{array}$ & $\begin{array}{l}\text { State- } \\
\text { Plane } \\
\text { Coord. } \\
\text { X (ft) }\end{array}$ & $\begin{array}{c}\text { State- } \\
\text { Plane } \\
\text { Coord. } \\
Y(f t)\end{array}$ & $\begin{array}{c}\text { Surface } \\
\text { Elevation } \\
\text { (ft) }\end{array}$ \\
\hline $\mathrm{H}-5 \mathrm{c}$ & $\begin{array}{l}05 / 22 / 78- \\
06 / 03 / 78\end{array}$ & HYD & $\begin{array}{l}\text { Used as a } \\
\text { hydrologic } \\
\text { monitoring well. }\end{array}$ & T22S, R31E, S15 & $1006 \mathrm{FNL}, 135 \mathrm{FEL}$ & 677,878 & 508,198 & 3505 \\
\hline $\mathrm{H}-6 \mathrm{a}$ & $\begin{array}{l}07 / 06 / 78- \\
07 / 11 / 78\end{array}$ & HYD & $\begin{array}{l}\text { Well used for } \\
\text { hydrologic } \\
\text { monitoring and } \\
\text { tracer testing. }\end{array}$ & T22S, R31E, S18 & $274 \mathrm{FWL}, 283 \mathrm{FNL}$ & 657,132 & 508,881 & 3347 \\
\hline$H-6 b$ & $\begin{array}{c}06 / 27 / 78- \\
07 / 05 / 78\end{array}$ & HYD & $\begin{array}{l}\text { Well used for } \\
\text { hydrologic } \\
\text { monitoring and } \\
\text { tracer testing. }\end{array}$ & T22S, R31E, S18 & $323 \mathrm{FWL}, 196 \mathrm{FNL}$ & 657,180 & 508,969 & 3348 \\
\hline$H-6 c$ & $\begin{array}{l}06 / 20 / 78- \\
06 / 26 / 78\end{array}$ & HYD & $\begin{array}{l}\text { Well used for } \\
\text { hydrologic } \\
\text { monitoring and } \\
\text { tracer testing. }\end{array}$ & T22S, R31E, S18 & 374 FWL, 281 FNL & 657,232 & 508,884 & 3348 \\
\hline $\mathrm{H}-7 \mathbf{a}$ & $\begin{array}{l}09 / 18 / 79- \\
09 / 22 / 79\end{array}$ & HYD & $\begin{array}{l}\text { Used as a } \\
\text { hydrologic } \\
\text { monitoring well. }\end{array}$ & T23S, R30E, S14 & 2495 FNL, 2492 FWL & 648,790 & 475,132 & 3164 \\
\hline $\mathrm{H}-7 \mathrm{~b} 1$ & $\begin{array}{c}09 / 13 / 79- \\
09 / 18 / 79\end{array}$ & HYD & $\begin{array}{l}\text { Used as a } \\
\text { hydrologic } \\
\text { monitoring well. }\end{array}$ & T23S, R30E, S14 & 2566 FNL, 2563 FWL & 648,862 & 475,061 & 3164 \\
\hline $\mathrm{H}-7 \mathrm{~b} 2$ & $\begin{array}{l}09 / 02 / 83- \\
09 / 21 / 83\end{array}$ & HYD & $\begin{array}{l}\text { Used as a } \\
\text { hydrologic } \\
\text { monitoring well. }\end{array}$ & T23S, R30E, S14 & 2662 FNL, 2538 FWL & 648,837 & 474,965 & 3164 \\
\hline $\mathrm{H}-7 \mathrm{c}$ & $\begin{array}{l}09 / 06 / 79- \\
09 / 13 / 79\end{array}$ & HYD & $\begin{array}{l}\text { Used as a } \\
\text { hydrologic } \\
\text { monitoring well. }\end{array}$ & T23S, R30E, S14 & 2592 FNL, 2468 FWL & 648,766 & 475,035 & 3163 \\
\hline$H-8 a$ & $\begin{array}{l}09 / 07 / 79- \\
09 / 18 / 79\end{array}$ & HYD & $\begin{array}{l}\text { Used as a } \\
\text { hydrologic } \\
\text { monitoring well. }\end{array}$ & T24S, R30E, S23 & 1963 FNL, 1487 FEL & 650,392 & 438,678 & 3433 \\
\hline$H-8 b$ & $\begin{array}{l}08 / 06 / 79- \\
08 / 12 / 79\end{array}$ & HYD & $\begin{array}{l}\text { Used as a } \\
\text { hydrologic } \\
\text { monitoring well. }\end{array}$ & T24S, R3OE, S23 & $1995 \mathrm{FNL}, 1405 \mathrm{FEL}$ & 650,473 & 438,646 & 3433 \\
\hline
\end{tabular}


Table 1. DOE-Sponsored Surface Boreholes at the Waste Isolation Pilot Plant (continued)

\begin{tabular}{|c|c|c|c|c|c|}
\hline ID & $\begin{array}{c}\text { Total } \\
\text { Depth }(\mathrm{ft})\end{array}$ & $\begin{array}{l}\text { Depth of } \\
\text { Cored } \\
\text { Interval(s) } \\
\text { (ft) }\end{array}$ &  & Reference(s) & Comments \\
\hline$H-5 \mathrm{C}$ & 1076 & $1026-1076$ & $\left\{2.2^{\prime \prime}\right\}$ & $\begin{array}{l}\text { USGS-WRI 82-19 (Dennehy and } \\
\text { Mercer, 1982), SAND82-0080 } \\
\text { (Seward, 1982), SAND85-7206 (Hydro } \\
\text { Geo Chem, Inc., 1985), SAND86-7166 } \\
\text { (Saulnier et al., 1986) }\end{array}$ & $\begin{array}{l}\text { Hole is completed in the Rustler/Salado contact and } \\
\text { the Culebra Dolomite. Hole bottomed in upper } \\
\text { member of Salado Formation. }\end{array}$ \\
\hline $\mathrm{H}-6 \mathrm{a}$ & 637 & $\begin{array}{l}475-525 \\
.\end{array}$ & $\left\{2.2^{\prime \prime}\right\}$ & $\begin{array}{l}\text { USGS-WRI 82-8 (Dennehy, 1982), } \\
\text { SAND82-0080 (Seward, 1982), } \\
\text { SAND85-7206 (Hydro Geo Chem, Inc., } \\
\text { 1985), SAND86-7166 (Saulnier et al., } \\
\text { 1986) }\end{array}$ & $\begin{array}{l}\text { Hole originally completed in the Magenta Dolomite, } \\
\text { later deepened to the lower member of Rustler } \\
\text { Formation. }\end{array}$ \\
\hline$H-6 b$ & 640 & $592-640$ & $\left\{2.2^{\prime \prime}\right\}$ & $\begin{array}{l}\text { USGS-WRI 82-8 (Dennehy, 1982), } \\
\text { SAND82-0080 (Seward, 1982), } \\
\text { SAND85-7206 (Hydro Geo Chem, Inc., } \\
\text { 1985), SAND86-7166 (Saulnier et al., } \\
\text { 1986) }\end{array}$ & $\begin{array}{l}\text { Hole is completed in Culebra Dolomite. Hole } \\
\text { bottomed in lower member of Rustler Formation. }\end{array}$ \\
\hline$H-6 c$ & 741 & $700-741$ & $\left\{2.2^{\prime \prime}\right\}$ & $\begin{array}{l}\text { USGS-WRI } 82-8 \text { (Dennehy, 1982), } \\
\text { SAND82-0080 (Seward, 1982), } \\
\text { SAND85-7206 (Hydro Geo Chem, Inc., } \\
\text { 1985), SAND86-7166 (Saulnier et al., } \\
\text { 1986) }\end{array}$ & $\begin{array}{l}\text { Hole is completed in Rustler/Salado contact \& the } \\
\text { Culebra Dolomite. Hole bottomed in upper member } \\
\text { of Salado Formation. }\end{array}$ \\
\hline $\mathrm{H}-7 \mathrm{a}$ & 154 & 114-154 & $\left\{3.5^{\prime \prime}\right\}$ & $\begin{array}{l}\text { USGS-WRI 82-38 (Drellack and Wells, } \\
\text { 1982a), SAND86-7109 (Intera } \\
\text { Technologies, Inc., 1986), SAND86- } \\
7166 \text { (Saulnier et al., 1986) }\end{array}$ & $\begin{array}{l}\text { Hole is completed in Magenta Dolomite. Hole } \\
\text { bottomed in Tamarisk member of Rustler Formation. }\end{array}$ \\
\hline $\mathrm{H}-7 \mathrm{~b} 1$ & 286 & $234-286$ & $\left\{3.5^{\prime \prime}\right\}$ & $\begin{array}{l}\text { USGS-WRI } 82-38 \text { (Drellack and Wells, } \\
\text { 1982a), SAND86-7109 (Intera } \\
\text { Technologies, Inc., 1986), SAND86- } \\
7166 \text { (Saulnier et al., 1986) }\end{array}$ & $\begin{array}{l}\text { Hole is completed in Culebra Dolomite. Hole } \\
\text { bottomed in lower member of Rustler Formation. }\end{array}$ \\
\hline $\mathrm{H}-7 \mathrm{~b} 2$ & 295 & $103-295$ & $\left\{3.5^{\prime \prime}\right\}$ & $\begin{array}{l}\text { SAND85-7206 (Hydro Geo Chem, Inc., } \\
\text { 1985), SAND86-7109 (Intera } \\
\text { Technologies, Inc., 1986, SAND86- } \\
\text { 7166 (Saulnier et al., 1986) }\end{array}$ & $\begin{array}{l}\text { Hole is completed in Culebra Dolomite to provide data } \\
\text { with which to evaluate transmissivity and, if possible, } \\
\text { the storativity of the Culebra member at the H-7 } \\
\text { hydropad. Hole bottomed in lower member of Rustler } \\
\text { Formation. }\end{array}$ \\
\hline $\mathrm{H}-7 \mathrm{C}$ & 420 & $\begin{array}{l}140-161 \\
203-292 \\
360-420\end{array}$ & $\begin{array}{l}\left\{3.5^{\prime}\right\} \\
\left\{3.5^{\prime}\right\} \\
\left\{3.5^{\prime \prime}\right\}\end{array}$ & $\begin{array}{l}\text { USGS-WRI 82-38 (Drellack and Wells, } \\
\text { 1982a), SAND86-7109 (Intera } \\
\text { Technologies, Inc., 1986), SAND86- } \\
7166 \text { (Saulnier et al., 1986) }\end{array}$ & $\begin{array}{l}\text { Hole is completed in Rustler/Salado contact. Hole } \\
\text { bottomed in upper member of Salado Formation. }\end{array}$ \\
\hline$H-8 a$ & 505 & $83-505$ & $\left\{3.5^{\prime \prime}\right\}$ & $\begin{array}{l}\text { USGS-WRI 82-4118 (Wells and } \\
\text { Drellack, 1982), SAND86-7109 (Intera } \\
\text { Technologies, Inc., 1986), SAND86- } \\
7166 \text { (Saulnier et al., 1986) }\end{array}$ & $\begin{array}{l}\text { Hole is completed in Magenta Dolomite. Hole } \\
\text { bottomed in Tamarisk member of Rustler Formation. }\end{array}$ \\
\hline$H-8 b$ & 624 & $576-624$ & $\left\{3.5^{\prime \prime}\right\}$ & $\begin{array}{l}\text { USGS-WRI 82-4118 (Wells and } \\
\text { Drellack, 1982), SAND86-7109 (Intera } \\
\text { Technologies, Inc., 1986), SAND86- } \\
7166 \text { (Saulnier et al., 1986) }\end{array}$ & $\begin{array}{l}\text { Hole is completed in Culebra Dolomite. Hole } \\
\text { bottomed in lower member of Rustler Formation. }\end{array}$ \\
\hline
\end{tabular}


Table 1. DOE-Sponsored Surface Boreholes at the Waste Isolation Pilot Plant (continued)

\begin{tabular}{|c|c|c|c|c|c|c|c|c|}
\hline ID & $\begin{array}{c}\text { Drilling } \\
\text { Start/End } \\
\text { Date }\end{array}$ & Purpose & Status & $\begin{array}{l}\text { Township/ } \\
\text { Rangel } \\
\text { Section }\end{array}$ & $\begin{array}{c}\text { Section } X \& Y \\
\text { Coordinates }\end{array}$ & $\begin{array}{l}\text { State- } \\
\text { Plane } \\
\text { Coord. } \\
X \text { (ft) }\end{array}$ & $\begin{array}{c}\text { State- } \\
\text { Plane } \\
\text { Coord. } \\
Y(\mathrm{ft})\end{array}$ & $\begin{array}{c}\text { Surface } \\
\text { Elevation } \\
\text { (ft) }\end{array}$ \\
\hline $\mathrm{H}-8 \mathrm{C}$ & $\begin{array}{l}07 / 27 / 79- \\
08 / 06 / 79\end{array}$ & HYD & $\begin{array}{l}\text { Used as a } \\
\text { hydrologic } \\
\text { monitoring well. }\end{array}$ & T24S, R30E, S23 & $2059 \mathrm{FNL}, 1470 \mathrm{FEL}$ & 650,409 & 438,581 & 3433 \\
\hline $\mathrm{H}-9 \mathrm{a}$ & $\begin{array}{l}07 / 09 / 79- \\
09 / 05 / 79\end{array}$ & HYD & $\begin{array}{l}\text { Used as a } \\
\text { hydrologic } \\
\text { monitoring well. }\end{array}$ & T24S, R31E, S4 & 2392 FNL, 139 FWL & 667,879 & 453,977 & 3405 \\
\hline $\begin{array}{c}\mathrm{H}-9 \mathrm{a} \\
\text { (deepened) }\end{array}$ & $\begin{array}{c}07 / 21 / 83- \\
07 / 27 / 83\end{array}$ & HYD & $\begin{array}{l}\text { Used as a } \\
\text { hydrologic. } \\
\text { monitoring well. }\end{array}$ & T24S, R31E, S4 & $2392 \mathrm{FNL}, 139 \mathrm{FWL}$ & 667,879 & 453,977 & 3405 \\
\hline$H-9 b$ & $\begin{array}{l}08 / 14 / 79- \\
08 / 28 / 79\end{array}$ & HYD & $\begin{array}{l}\text { Used as a } \\
\text { hydrologic } \\
\text { monitoring well. }\end{array}$ & T24S, R31E, S4 & $2391 \mathrm{FNL}, 239 \mathrm{FWL}$ & 667,979 & 453,978 & 3406 \\
\hline $\mathrm{H}-9 \mathrm{c}$ & $\begin{array}{c}08 / 01 / 79- \\
08 / 24 / 79\end{array}$ & HYD & $\begin{array}{l}\text { Used as a } \\
\text { hydrologic } \\
\text { monitoring well. }\end{array}$ & T24S, R31E, S4 & $2479 \mathrm{FNL}, 188 \mathrm{FWL}$ & 667,929 & 453,890 & 3406 \\
\hline$H-10 a$ & $\begin{array}{c}08 / 21 / 79- \\
08 / 26 / 79\end{array}$ & HYD & $\begin{array}{l}\text { Used as a } \\
\text { hydrologic } \\
\text { monitoring well. }\end{array}$ & T23S, R32E, S20 & 433 FSL, 2069 FEL & 697,463 & 467,561 & 3687 \\
\hline $\mathrm{H}-10 \mathrm{~b}$ & $\begin{array}{c}10 / 07 / 79- \\
10 / 13 / 79\end{array}$ & HYD & $\begin{array}{l}\text { Used as a } \\
\text { hydrologic } \\
\text { monitoring well. }\end{array}$ & T23S, R32E, S20 & $485 \mathrm{FSL}, 1982 \mathrm{FEL}$ & 697,549 & 467,613 & 3687 \\
\hline$H-10 c$ & $\begin{array}{c}08 / 11 / 79- \\
08 / 20 / 79\end{array}$ & HYD & $\begin{array}{l}\text { Used as a } \\
\text { hydrologic } \\
\text { monitoring well. }\end{array}$ & T23S, R32E, S20 & $385 \mathrm{FSL}, 1982 \mathrm{FEL}$ & 697,550 & 467,513 & 3687 \\
\hline $\mathrm{H}-11 \mathrm{~b} 1$ & $\begin{array}{c}08 / 03 / 83- \\
09 / 02 / 83\end{array}$ & $\begin{array}{l}\text { HYD, } \\
\text { MULTI }\end{array}$ & $\begin{array}{l}\text { Well used for } \\
\text { hydrologic } \\
\text { monitoring and } \\
\text { tracer testing. }\end{array}$ & T22S, R31E, S33 & 1511 FSL, 174 FEL & 672,647 & 489,617 & 3411 \\
\hline
\end{tabular}


Table 1. DOE-Sponsored Surface Boreholes at the Waste Isolation Pilot Plant (continued)

\begin{tabular}{|c|c|c|c|c|c|}
\hline ID & $\begin{array}{c}\text { Total } \\
\text { Depth }(\mathrm{ft})\end{array}$ & $\begin{array}{l}\text { Depth of } \\
\text { Cored } \\
\text { Interval(s) } \\
\text { (ft) }\end{array}$ & $\begin{array}{l}\text { 荘 } \\
0 \\
0 \\
0 \\
0\end{array}$ & Reference(s) & Comments \\
\hline $\mathrm{H}-8 \mathrm{C}$ & 808 & $463-808$ & $\left\{3.5^{\prime \prime}\right\}$ & $\begin{array}{l}\text { USGS-WRI 82-4118 Wells and } \\
\text { Drellack, 1982), SAND86-7109 (Intera } \\
\text { Technologies, Inc., 1986), SAND86- } \\
7166 \text { (Saulnier et al., 1986) }\end{array}$ & $\begin{array}{l}\text { Hole is completed in Rustler/Salado contact. Hole } \\
\text { bottomed in upper member of Salado Formation. }\end{array}$ \\
\hline $\mathrm{H}-9 \mathrm{a}$ & 559 & $514-559$ & $\left\{3.5^{\prime \prime}\right\}$ & $\begin{array}{l}\text { USGS-WRI 82-4111 (Drellack and } \\
\text { Wells, 1982b), SAND85-7206 (Hydro } \\
\text { Geo Chem, Inc., 1985), SAND85-7263 } \\
\text { (Intera Technologies, Inc., and Hydro } \\
\text { Geo Chem, Inc.; 1985) }\end{array}$ & $\begin{array}{l}\text { Hole is completed in Magenta Dolomite. Hole } \\
\text { bottomed in Tamarisk member of Rustler Formation. }\end{array}$ \\
\hline $\begin{array}{c}\mathrm{H}-9 \mathrm{a} \\
\text { (deepened) }\end{array}$ & 692 & $616-689$ & $\left\{2.2^{\prime \prime}\right\}$ & $\begin{array}{l}\text { SAND85-7206 (Hydro Geo Chem, Inc., } \\
\text { 1985), SAND85-7263 (Intera } \\
\text { Technologies, inc., and Hydro Geo } \\
\text { Chem, Inc., 1985), SAND86-7166 } \\
\text { (Saulnier et al., 1986) }\end{array}$ & $\begin{array}{l}\text { Hole deepened and recompleted in Culebra. Hole } \\
\text { bottomed in lower member of Rustler Formation. }\end{array}$ \\
\hline$H-9 b$ & 708 & $640-680$ & $\left\{3.5^{\prime \prime}\right\}$ & $\begin{array}{l}\text { USGS-WRI 82-4111 (Drellack and } \\
\text { Wells, 1982b), SAND85-7206 (Hydro } \\
\text { Geo Chem, Inc., 1985), SAND85-7263 } \\
\text { (Intera Technologies, Inc., and Hydro } \\
\text { Geo Chem, Inc., 1985), SAND86-7166 } \\
\text { (Saulnier et al., 1986) }\end{array}$ & $\begin{array}{l}\text { Hole is completed in Culebra Dolomite. Hole } \\
\text { bottomed in lower member of Rustler Formation. }\end{array}$ \\
\hline$H-9 c$ & 816 & $\begin{array}{l}505-562 \\
620-681 \\
789-816\end{array}$ & $\begin{array}{l}\left\{3.5^{5}\right\} \\
\left\{3.5^{\prime \prime}\right\} \\
\left\{3.5^{n}\right\}\end{array}$ & $\begin{array}{l}\text { USGS-WRI 82-4111 (Drellack and } \\
\text { Wells, 1982b), SAND85-7206 (Hydro } \\
\text { Geo Chem, Inc., 1985), SAND85-7263 } \\
\text { (Intera Technologies, Inc., and Hydro } \\
\text { Geo Chem, Inc., 1985), SAND86-7166 } \\
\text { (Saulnier et al., 1986) }\end{array}$ & $\begin{array}{l}\text { Hole is completed in the Rustler/Salado contact and } \\
\text { the Culebra Dolomite. Hole bottomed in upper } \\
\text { member of Salado Formation. }\end{array}$ \\
\hline $\mathrm{H}-10 \mathrm{a}$ & 1318 & $1247-1287$ & $\left\{3.5^{\prime \prime}\right\}$ & $\begin{array}{l}\text { USGS-WRI 83-4124 Wells and } \\
\text { Drellack, 1983), SAND86-7166 } \\
\text { (Sauinier et al., 1986) }\end{array}$ & $\begin{array}{l}\text { Hole is completed in Magenta Dolomite. Hole } \\
\text { bottomed in Tamarisk member of Rustler Formation. }\end{array}$ \\
\hline $\mathrm{H}-10 \mathrm{~b}$ & 1398 & $\begin{array}{c}639-659 \\
1348-1398\end{array}$ & $\begin{array}{l}\left\{3.5^{\prime \prime}\right\} \\
\left\{3.5^{\prime \prime}\right\}\end{array}$ & $\begin{array}{l}\text { USGS-WRI 83-4124 Wells and } \\
\text { Drellack, 1983), SAND86-7166 } \\
\text { (Saulnier et al., 1986) }\end{array}$ & $\begin{array}{l}\text { Hole is completed in Culebra Dolomite. Hole } \\
\text { bottomed in lower member of Rustler Formation. }\end{array}$ \\
\hline$H-10 c$ & $1538^{*}$ & $\begin{array}{l}1230-1281 \\
1346-1394 \\
1489-1518\end{array}$ & $\begin{array}{l}\left\{3.5^{\prime \prime}\right\} \\
\left\{3.5^{\prime \prime}\right\} \\
\left\{3.5^{\prime \prime}\right\}\end{array}$ & $\begin{array}{l}\text { USGS-WRI 83-4124 (Wells and } \\
\text { Drellack, 1983), SAND86-7166 } \\
\text { (Saulnier et al., 1986) }\end{array}$ & $\begin{array}{l}\text { Hole is completed in the Rustler/Salado contact. Hole } \\
\text { bottomed in upper member of Salado Formation. }\end{array}$ \\
\hline $\mathrm{H}-11 \mathrm{~b} 1$ & 785 & $210-785$ & $\left\{2.2^{\prime \prime}\right\}$ & $\begin{array}{l}\text { SAND85-7206 (Hydro Geo Chem, } \\
\text { Inc., 1985), SAND85-7263 (Intera } \\
\text { Technologies, InC., and Hydro Geo } \\
\text { Chem, Inc., 1985), SAND86-7166 } \\
\text { (Saulnier et al., 1986), SAND89- } \\
0200 \text { (Mercer and Snyder, 1990a), } \\
\text { SAND89-7056 (Stensrud et al., } \\
\text { 1990), Sanchez and McCasland } \\
\text { (1994) }\end{array}$ & $\begin{array}{l}\text { Drilled to investigate stratigraphy and hydrology of } \\
\text { the Culebra. Evaluations in } 1983 \text { indicated no } \\
\text { evidence of groundwater movement along the } \\
\text { contact of the Rustler Formation with the overlying } \\
\text { Dewey Lake Red Beds. Halite deposits } 20 \mathrm{ft} \text { below } \\
\text { base of Culebra. Hole bottomed in lower member } \\
\text { of the Rustler Formation. }\end{array}$ \\
\hline
\end{tabular}


Table 1. DOE-Sponsored Surface Boreholes at the Waste Isolation Pilot Plant (continued)

\begin{tabular}{|c|c|c|c|c|c|c|c|c|}
\hline ID & $\begin{array}{c}\text { Drilling } \\
\text { Start/End } \\
\text { Date }\end{array}$ & Purpose & Status & $\begin{array}{l}\text { Township/ } \\
\text { Rangel } \\
\text { Section }\end{array}$ & $\begin{array}{l}\text { Section } X \& Y \\
\text { Coordinates }\end{array}$ & $\begin{array}{l}\text { State- } \\
\text { Plane } \\
\text { Coord. } \\
X \text { (ft) }\end{array}$ & $\begin{array}{c}\text { State- } \\
\text { Plane } \\
\text { Coord. } \\
Y \text { (ft) }\end{array}$ & $\begin{array}{c}\text { Surface } \\
\text { Elevation } \\
\text { (ft) }\end{array}$ \\
\hline $\mathrm{H}-11 \mathrm{~b} 2$ & $\begin{array}{c}10 / 01 / 83- \\
11 / 28 / 83\end{array}$ & $\begin{array}{l}\text { HYD, } \\
\text { MULTI }\end{array}$ & $\begin{array}{l}\text { Well used for } \\
\text { hydrologic } \\
\text { monitoring and } \\
\text { tracer testing. }\end{array}$ & T22S, R31E, S33 & 1436 FSL, 169 FEL & 672,653 & 489,542 & 3411 \\
\hline $\mathrm{H}-11 \mathrm{b3}$ & $\begin{array}{l}12 / 01 / 83 \\
01 / 04 / 84\end{array}$ & $\begin{array}{l}\text { HYD, } \\
\text { MULTI }\end{array}$ & $\begin{array}{l}\text { Well used for } \\
\text { hydrologic } \\
\text { monitoring and } \\
\text { tracer testing. }\end{array}$ & T22S, R31E, S33 & $1502 \mathrm{FSL}, 105 \mathrm{FEL}$ & 672,716 & 489,608 & 3412 \\
\hline $\mathrm{H}-11 \mathrm{bA}$ & $\begin{array}{c}02 / 23 / 88 \\
03 / 15 / 88\end{array}$ & $\begin{array}{l}\text { HYD, } \\
\text { MULTI }\end{array}$ & $\begin{array}{l}\text { Well used for } \\
\text { hydrologic } \\
\text { monitoring and } \\
\text { tracer testing. }\end{array}$ & T22S, R31E, S33 & $1515 \mathrm{FSL}, 320 \mathrm{FEL}$ & 672,501 & 489,620 & 3410 \\
\hline $\mathrm{H}-12$ & $\begin{array}{c}10 / 04 / 83- \\
10 / 18 / 83\end{array}$ & HYD & $\begin{array}{l}\text { Used as a } \\
\text { hydrologic } \\
\text { monitoring well. }\end{array}$ & T23S, R31E, S15 & $23 \mathrm{FNL}, 92 \mathrm{FEL}$ & 678,079 & 477,535 & 3426 \\
\hline$H-14$ & $\begin{array}{c}09 / 25 / 86- \\
10 / 23 / 86\end{array}$ & HYD & $\begin{array}{l}\text { Used as a } \\
\text { hydrologic } \\
\text { monitoring well. }\end{array}$ & T22S, R31E, S29 & 372 FSL, 562 FWL & 662,815 & 493,697 & 3346 \\
\hline$H-15$ & $\begin{array}{c}10 / 24 / 86- \\
11 / 14 / 86\end{array}$ & HYD & $\begin{array}{l}\text { Used as a } \\
\text { hydrologic } \\
\text { monitoring well. }\end{array}$ & T22S, 31E, S28 & 89 FNL, 174 FEL & 672,606 & 498,572 & 3480 \\
\hline$H-16$ & $\begin{array}{l}07 / 13 / 87- \\
08 / 18 / 87\end{array}$ & HYD & $\begin{array}{l}\text { Used as a } \\
\text { hydrologic } \\
\text { monitoring well. }\end{array}$ & T22S, R31E, S20 & $1113 \mathrm{FSL}, 1241 \mathrm{FEL}$ & 666,231 & 499,726 & 3410 \\
\hline
\end{tabular}


Table 1. DOE-Sponsored Surface Boreholes at the Waste Isolation Pilot Plant (continued)

\begin{tabular}{|c|c|c|c|c|c|}
\hline ID & $\begin{array}{c}\text { Total } \\
\text { Depth }(\mathrm{ft})\end{array}$ & $\begin{array}{l}\text { Depth of } \\
\text { Cored } \\
\text { Interval(s) } \\
\text { (ft) }\end{array}$ & $\begin{array}{l}\text { 颉 } \\
\stackrel{0}{0} \\
\stackrel{0}{0}\end{array}$ & Reference(s) & Comments \\
\hline$H-11 b 2$ & 776 & $\begin{array}{c}50-75 \\
145-220 \\
420-721\end{array}$ & $\begin{array}{l}\left\{2.2^{\prime \prime}\right\} \\
\left\{2.2^{\prime \prime}\right\} \\
\left\{2.2^{\prime \prime}\right\}\end{array}$ & $\begin{array}{l}\text { SAND85-7206 (Hydro Geo Chem, } \\
\text { Inc., 1985), SAND85-7263 (intera } \\
\text { Technologies, Inc., and Hydro Geo } \\
\text { Chem, Inc., 1985), SAND86-7166 } \\
\text { (Saulnier et al., 1986), SAND89- } \\
\text { O200 (Mercer and Snyder, 1990a), } \\
\text { SAND89-7056 (Stensrud et al., } \\
\text { 1990). Sanchez and McCasland } \\
\text { (1994) }\end{array}$ & $\begin{array}{l}\text { Drilled to investigate stratigraphy and hydrology of } \\
\text { the Culebra. Evaluations in } 1983 \text { indicated no } \\
\text { evidence of groundwater movement along the } \\
\text { contact of the Rustler Formation with the overtying } \\
\text { Dewey Lake Red Beds. Halite deposits } 20 \mathrm{ft} \text { below } \\
\text { base of Culebra. Hole bottomed in lower member } \\
\text { of Rustler Formation. }\end{array}$ \\
\hline $\mathrm{H}-11 \mathrm{b3}$ & 789 & $60-789$ & $\left\{2.2^{\prime \prime}\right\}$ & $\begin{array}{l}\text { SAND85-7263 (Intera } \\
\text { Technologies, Inc., and Hydro Geo } \\
\text { Chem, Inc., 1985), SAND86-7109 } \\
\text { (intera Technologies, Inc., 1986), } \\
\text { SAND86-7166 (Saulnier et al., } \\
\text { 1986), SAND89-0200 (Mercer and } \\
\text { Snyder, 1990a), SAND89-7056 } \\
\text { (Stensrud et al., 1990), Sanchez } \\
\text { and McCasland (1994) }\end{array}$ & $\begin{array}{l}\text { Drilled to investigate stratigraphy and hydrology of } \\
\text { the Culebra. Evaluations in } 1983 \text { indicated no } \\
\text { evidence of groundwater movement along the } \\
\text { contact of the Rustler Formation with the overlying } \\
\text { Dewey Lake Red Beds. Halite deposits } 20 \mathrm{ft} \text { below } \\
\text { base of Culebra. Hole bottomed in lower member of } \\
\text { Rustler Formation. }\end{array}$ \\
\hline $\mathrm{H}-11 \mathrm{~b} 4$ & 765 & $715-765$ & $\left\{2.2^{\prime \prime}\right\}$ & $\begin{array}{l}\text { SAND85-7263 (Intera Technologies, } \\
\text { Inc., and Hydro Geo Chem, Inc., } \\
\text { 1985), SAND86-7166 (Saulnier et } \\
\text { al., 1986), SAND88-7014 (Stensrud } \\
\text { et al., 1988b), SAND89-0200 } \\
\text { (Mercer and Snyder, 1990a), SAND } \\
\text { 89-0536 (Beauheim, 1989), } \\
\text { SAND89-7056 (Stensrud et al., } \\
\text { 1990), Sanchez and McCasland } \\
\text { (1994) }\end{array}$ & $\begin{array}{l}\text { Drilled to provide additional stratigraphic info on } \\
\text { Dewey Lake Red Beds \& upper four members of } \\
\text { Rustler Formation, to provide hydraulic-property } \\
\text { cata on Culebra Dolomite, \& also to provide site } \\
\text { for long-term monitoring of Culebra response to } \\
\text { ongoing hydrologic testing. Hole bottomed in } \\
\text { lower member of Rustler Formation. }\end{array}$ \\
\hline $\mathrm{H}-12$ & 1001 & $\begin{array}{l}129-154 \\
274-314 \\
630-1,001\end{array}$ & $\begin{array}{l}\left\{2.2^{\prime \prime}\right\} \\
\left\{2.2^{\prime \prime}\right\} \\
\left\{2.2^{\prime \prime}\right\}\end{array}$ & $\begin{array}{l}\text { SAND85-7206 (Hydro Geo Chem, Inc., } \\
\text { 1985), SAND85-7263 (Intera } \\
\text { Technologies, Inc., and Hydro Geo } \\
\text { Chem, Inc., 1985), SAND87-7166 } \\
\text { (Stensrud et al., 1988), SAND89-0201 } \\
\text { (Mercer and Snyder, 1990b) }\end{array}$ & $\begin{array}{l}\text { Drilled to refine and quantify the hydrologic model for } \\
\text { Rustler Formation \& investigate the extent of solution } \\
\text { of halite in Rustler Formation. Hole bottomed in } \\
\text { upper member of Salado Formation. }\end{array}$ \\
\hline $\mathrm{H}-14$ & 589 & $\begin{array}{l}422-451 \\
535-574\end{array}$ & $\begin{array}{l}\left\{2.2^{\prime \prime}\right\} \\
\left\{2.2^{m}\right\}\end{array}$ & $\begin{array}{l}\text { SAND87-7125 (Stensrud et al., 1987), } \\
\text { SAND89-0202 (Mercer and Snyder, } \\
\text { 1990C), Sanchez and McCasland } \\
\text { (1994) }\end{array}$ & $\begin{array}{l}\text { Continuous cores taken through Magenta \& Culebra } \\
\text { members of Rustler \& across their upper \& lower } \\
\text { contacts. Hole bottomed in lower member of Rustler } \\
\text { Formation. }\end{array}$ \\
\hline $\mathrm{H}-15$ & 900 & $\begin{array}{l}744-774 \\
855-891\end{array}$ & $\begin{array}{l}\left\{2.2^{\prime \prime}\right\} \\
\left\{2.2^{\prime \prime}\right\}\end{array}$ & $\begin{array}{l}\text { SAND87-7125 (Stensrud et al., 1987), } \\
\text { SAND89-0202 (Mercer and Snyder, } \\
\text { 1990c) }\end{array}$ & $\begin{array}{l}\text { Continuous cores taken through Magenta \& } \\
\text { Culebra Dolomites \& across upper \& lower } \\
\text { contacts. No halite observed in Rustler Formation } \\
\text { cores \& cuttings. Culebra Member not fractured. } \\
\text { Hole bottomed in lower member of Rustler } \\
\text { Formation. }\end{array}$ \\
\hline $\mathrm{H}-16$ & 851 & 474-851 & $\left\{2.2^{\prime \prime}\right\}$ & $\begin{array}{l}\text { SAND87-7166 (Stensrud et al., 1988a), } \\
\text { SAND89-0203 (Mercer and Snyder, } \\
\text { 1990d) }\end{array}$ & $\begin{array}{l}\text { Drilled to assess pre- \& post-mining hydrology } \\
\text { associated with fourth shaft (air-intake) at WPP. } \\
\text { Intervals in Rustler Formation were cored \& } \\
\text { hydraulically tested. Hole bottomed in upper } \\
\text { member of the Salado Formation. }\end{array}$ \\
\hline
\end{tabular}


Table 1. DOE-Sponsored Surface Boreholes at the Waste Isolation Pilot Plant (continued)

\begin{tabular}{|c|c|c|c|c|c|c|c|c|}
\hline ID & $\begin{array}{l}\text { Drilling } \\
\text { Start/End } \\
\text { Date }\end{array}$ & Purpose & Status & $\begin{array}{c}\text { Township/ } \\
\text { Range/ } \\
\text { Section }\end{array}$ & $\begin{array}{c}\text { Section X \& Y } \\
\text { Coordinates }\end{array}$ & $\begin{array}{l}\text { State- } \\
\text { Plane } \\
\text { Coord. } \\
\text { X (ft) }\end{array}$ & $\begin{array}{l}\text { State- } \\
\text { Plane } \\
\text { Coord. } \\
\text { Y (ft) }\end{array}$ & $\begin{array}{c}\text { Surface } \\
\text { Elevation } \\
\text { (ft) }\end{array}$ \\
\hline $\mathrm{H}-17$ & $\begin{array}{c}09 / 21 / 87- \\
11 / 04 / 87\end{array}$ & HYD & $\begin{array}{l}\text { Used as a } \\
\text { hydrologic } \\
\text { monitoring well. }\end{array}$ & T23S, R31E, S3 & 1466 FSL, 994 FWL & 673,837 & 484,304 & 3384 \\
\hline $\mathrm{H}-18$ & $\begin{array}{c}09 / 29 / 87- \\
11 / 18 / 87\end{array}$ & HYD & $\begin{array}{l}\text { Used as a } \\
\text { hydrologic } \\
\text { monitoring well. }\end{array}$ & T22S, R31E, S20 & $965 \mathrm{FNL}, 446 \mathrm{FWL}$ & 662,621 & 502,926 & 3413 \\
\hline$H-19 b 0$ & $\begin{array}{l}03 / 28 / 95- \\
04 / 23 / 95\end{array}$ & HYD & $\begin{array}{l}\text { Monitoring } \\
\text { hydrologic tracer } \\
\text { tests. }\end{array}$ & T22S, R31E, S28 & $1485 \mathrm{FSL}, 2460 \mathrm{FWL}$ & 669,964 & 494,836 & 3417 \\
\hline$H-19 b 1$ & $\begin{array}{l}02 / 13 / 95- \\
03 / 21 / 95\end{array}$ & HYD & Plugged & T22S, R31E, S28 & 1535 FSL, $2461 \mathrm{FWL}$ & 669,964 & 494,886 & 3417 \\
\hline $\mathrm{H}-19 \mathrm{~b} 2$ & $\begin{array}{l}05 / 10 / 95- \\
05 / 20 / 95\end{array}$ & HYD & $\begin{array}{l}\text { Monitoring } \\
\text { hydrologic tracer } \\
\text { tests. }\end{array}$ & T22S, R31E, S28 & $1434 \mathrm{FSL}, 2460 \mathrm{FMR}$ & 669,963 & 494,786 & 3417 \\
\hline$H-19 b 3$ & $\begin{array}{l}04 / 23 / 95- \\
05 / 09 / 95\end{array}$ & HYD & $\begin{array}{l}\text { Monitoring } \\
\text { hydrologic tracer } \\
\text { tests. }\end{array}$ & T22S, R31E, S28 & $1509 \mathrm{FSL}, 2504 \mathrm{FWL}$ & 670007 & 494,861 & 3417 \\
\hline$H-19 b 4$ & $\begin{array}{l}05 / 20 / 95- \\
06 / 05 / 95\end{array}$ & HYD & $\begin{array}{l}\text { Monitoring } \\
\text { hydrologic tracer } \\
\text { tests. }\end{array}$ & T22S, R31E, S28 & 1511 FSL, 2417 FWL & 669,920 & 494,861 & 3416 \\
\hline
\end{tabular}


Table 1. DOE-Sponsored Surface Boreholes at the Waste Isolation Pilot Plant (continued)

\begin{tabular}{|c|c|c|c|c|c|}
\hline ID & $\begin{array}{c}\text { Total } \\
\text { Depth }(\mathrm{ft})\end{array}$ & $\begin{array}{l}\text { Depth of } \\
\text { Cored } \\
\text { Interval(s) } \\
\text { (ft) }\end{array}$ & $\begin{array}{l}\text { 寄 } \\
0 \\
0 \\
0 \\
0\end{array}$ & Reference(s) & Comments \\
\hline $\mathrm{H}-17$ & 880 & $510-870$ & $\left\{2.2^{n \prime}\right\}$ & $\begin{array}{l}\text { SAND87-7166 (Stensrud et al., 1988a), } \\
\text { SAND89-0204 (Mercer and Snyder, } \\
\text { 1990e) }\end{array}$ & $\begin{array}{l}\text { Drilled to assess uncertainties in site hydrologic } \\
\text { parameters \& serve as monitoring points for planned } \\
\text { hydrologic tests. Halite above \& below Culebra } \\
\text { Dolomite Member of Rustler. Hole bottomed in upper } \\
\text { member of Salado Formation. }\end{array}$ \\
\hline$H-18$ & 840 & $40-830$ & $\left\{2.2^{\prime \prime}\right\}$ & $\begin{array}{l}\text { SAND87-7166 (Stensrud et al., 1988a), } \\
\text { SAND88-7014 (Stensrud et al., 1988b), } \\
\text { SAND89-0204 (Mercer and Snyder, } \\
\text { 1990e) }\end{array}$ & $\begin{array}{l}\text { Drilled to perform hydrologic tests on Culebra } \\
\text { Dolomite Member, Rustler Formation. First halite } \\
\text { beds were encountered below Culebra. Hole } \\
\text { bottomed in upper member of Salado Formation. }\end{array}$ \\
\hline$H-19 b 0$ & 779 & $741-779$ & $\left\{5.9^{\prime \prime}\right\}$ & $\begin{array}{l}\text { Appendix } A^{4} \text {; Technical Memorandum } \\
\text { (Mercer, 1996) }\end{array}$ & $\begin{array}{l}\text { Drilled to conduct tracer tests to define the nature of } \\
\text { solute transport in the Culebra Dolomite \& provide } \\
\text { quantitative estimates of transport parameters } \\
\text { needed for Performance Assessment calculations. } \\
\text { Geologic \& geophysical data were also obtained. } \\
\text { Hole bottomed in lower member of Rustler } \\
\text { Formation. }\end{array}$ \\
\hline$H-19 b 1$ & 733 & $37-733$ & $\left\{3.3^{\prime \prime}\right\}$ & $\begin{array}{l}\text { Appendix } A^{4} \text {; Technical Memorandum } \\
\text { (Mercer, 1996) }\end{array}$ & $\begin{array}{l}\text { Drilled to conduct tracer tests to define the nature of } \\
\text { solute transport in the Culebra Dolomite \& provide } \\
\text { quantitative estimates of transport parameters } \\
\text { needed for Performance Assessment calculations. } \\
\text { Geologic \& geophysical data were also obtained. } \\
\text { Tools dropped in hole; thus, hole was abandoned. } \\
\text { Hole bottomed in Tamarisk member of Rustler } \\
\text { Formation. }\end{array}$ \\
\hline $\mathrm{H}-19 \mathrm{~b} 2$ & 785 & $736-765$ & $\left\{3.3^{\prime \prime}\right\}$ & $\begin{array}{l}\text { Appendix A4; Technical Memorandum } \\
\text { (Mercer, 1996) }\end{array}$ & $\begin{array}{l}\text { Drilled to conduct tracer tests to define the nature of } \\
\text { solute transport in the Culebra Dolomite \& provide } \\
\text { quantitative estimates of transport parameters } \\
\text { needed for Performance Assessment calculations. } \\
\text { Geologic \& geophysical data were also obtained. } \\
\text { Hole bottomed in lower member of Rustler } \\
\text { Formation. }\end{array}$ \\
\hline $\mathrm{H}-19 \mathrm{b3}$ & 785 & $735-768$ & $\left\{3.3^{\prime \prime}\right\}$ & $\begin{array}{l}\text { Appendix A4; Technical Memorandum } \\
\text { (Mercer, 1996) }\end{array}$ & $\begin{array}{l}\text { Drilled to conduct tracer tests to define the nature of } \\
\text { solute transport in the Culebra Dolomite \& provide } \\
\text { quantitative estimates of transport parameters } \\
\text { needed for Performance Assessment calculations. } \\
\text { Geologic \& geophysical data were also obtained. } \\
\text { Hole bottomed in lower member of Rustler } \\
\text { Formation. }\end{array}$ \\
\hline $\mathrm{H}-19 \mathrm{~b} 4$ & 782 & $736-782$ & $\left\{3.3^{\prime \prime}\right\}$ & $\begin{array}{l}\text { Appendix A4; Technical Memorandum } \\
\text { (Mercer, 1996) }\end{array}$ & $\begin{array}{l}\text { Drilled to conduct tracer tests to define the nature of } \\
\text { solute transport in the Culebra Dolomite \& provide } \\
\text { quantitative estimates of transport parameters } \\
\text { needed for Performance Assessment calculations. } \\
\text { Geologic \& geophysical data were also obtained. } \\
\text { Hole bottomed in lower member of Rustler } \\
\text { Formation. }\end{array}$ \\
\hline
\end{tabular}


Table 1. DOE-Sponsored Surface Boreholes at the Waste Isolation Pilot Plant (continued)

\begin{tabular}{|c|c|c|c|c|c|c|c|c|}
\hline ID & $\begin{array}{l}\text { Drilling } \\
\text { Start/End } \\
\text { Date }\end{array}$ & Purpose & Status & $\begin{array}{l}\text { Township/ } \\
\text { Range/ } \\
\text { Section }\end{array}$ & $\begin{array}{l}\text { Section } X \& Y \\
\text { Coordinates }\end{array}$ & $\begin{array}{l}\text { State- } \\
\text { Plane } \\
\text { Coord. } \\
X \text { (ft) }\end{array}$ & $\begin{array}{c}\text { State- } \\
\text { Plane } \\
\text { Coord. } \\
Y(f t)\end{array}$ & $\begin{array}{c}\text { Surface } \\
\text { Elevation } \\
\text { (ft) }\end{array}$ \\
\hline $\mathrm{H}-19 \mathrm{b5}$ & $\begin{array}{l}06 / 11 / 95- \\
07 / 06 / 95\end{array}$ & HYD & $\begin{array}{l}\text { Monitoring } \\
\text { hydrologic tracer } \\
\text { tests. }\end{array}$ & T22S, R31E, S28 & $1466 \mathrm{FSL}, 2420 \mathrm{FWL}$ & 669,924 & 494,817 & 3417 \\
\hline $\begin{array}{c}\mathrm{H}-19 \mathrm{b5} \\
\text { (deepened) }\end{array}$ & $\begin{array}{l}08 / 25 / 95- \\
08 / 26 / 95\end{array}$ & HYD & $\begin{array}{l}\text { Monitoring } \\
\text { hydrologic tracer } \\
\text { tests. }\end{array}$ & T22S, R31E, S28 & 1466 FSL, 2420 FWL & 669,924 & 494,817 & 3417 \\
\hline$H-1966$ & $\begin{array}{l}07 / 10 / 95 \\
07 / 26 / 95\end{array}$ & HYD & $\begin{array}{l}\text { Monitoring } \\
\text { hydrologic tracer } \\
\text { tests. }\end{array}$ & T22S, R31E, S28 & 1554 FSL, 2473 FWL & 669,975 & 494,906 & 3417 \\
\hline $\begin{array}{c}\text { H-19b6 } \\
\text { (deepened) }\end{array}$ & $\begin{array}{l}08 / 23 / 95- \\
08 / 24 / 95\end{array}$ & HYD & $\begin{array}{l}\text { Monitoring } \\
\text { hydrologic tracer } \\
\text { tests. }\end{array}$ & T22S, R31E, S2B & 1554 FSL, 2473 FWL & 669,975 & 494,906 & 3417 \\
\hline$H-19 b 7$ & $\begin{array}{l}07 / 26 / 95- \\
08 / 18 / 95\end{array}$ & HYD & $\begin{array}{l}\text { Monitoring } \\
\text { hydrologic tracer } \\
\text { tests. }\end{array}$ & T22S, R31E, S28 & $1456 \mathrm{FSL}, 2464 \mathrm{FWL}$ & 669,967 & 494,807 & 3417 \\
\hline WQSP-1 & $\begin{array}{l}09 / 13 / 94- \\
09 / 16 / 94\end{array}$ & HYD & $\begin{array}{l}\text { Monitoring water } \\
\text { quality. }\end{array}$ & T22S, R31E, S16 & $115 \mathrm{FNL}, 1404 \mathrm{FWL}$ & 663,594 & 503,787 & 3417 \\
\hline WQSP-2 & $\begin{array}{l}09 / 06 / 94- \\
09 / 10 / 94\end{array}$ & HYD & $\begin{array}{l}\text { Monitoring water } \\
\text { quality. }\end{array}$ & T22S, R31E, S16 & 1611 FSL, $118 \mathrm{FML}$ & 667,580 & 505,540 & 3461 \\
\hline WQSP-3 & $\begin{array}{l}10 / 20 / 94- \\
10 / 26 / 94\end{array}$ & HYD & $\begin{array}{l}\text { Monitoring water } \\
\text { quality. }\end{array}$ & T22S, R31E, S16 & 54 FSL, 2208 FEL & 670,574 & 503,993 & 3477 \\
\hline
\end{tabular}


Table 1. DOE-Sponsored Surface Boreholes at the Waste Isolation Pilot Plant (continued)

\begin{tabular}{|c|c|c|c|c|c|}
\hline ID & $\begin{array}{c}\text { Total } \\
\text { Depth }(\mathrm{ft})\end{array}$ & $\begin{array}{l}\text { Depth of } \\
\text { Cored } \\
\text { Interval(s) } \\
\text { (ft) }\end{array}$ & 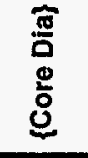 & Reference(s) & Comments \\
\hline $\mathrm{H}-19 \mathrm{~b} 5$ & 736 & $\begin{array}{l}\text { Not } \\
\text { Cored }\end{array}$ & & $\begin{array}{l}\text { Appendix A4; Technical Memorandum } \\
\text { (Mercer, 1996) }\end{array}$ & $\begin{array}{l}\text { Drilled to conduct tracer tests to define the nature of } \\
\text { solute transport in the Culebra Dolomite \& provide } \\
\text { quantitative estimates of transport parameters } \\
\text { needed for Performance Assessment calculations. } \\
\text { Geologic \& geophysical data were also obtained. } \\
\text { Hole bottomed in Tamarisk member of Rustler } \\
\text { Formation. }\end{array}$ \\
\hline $\begin{array}{c}\mathrm{H}-19 \mathrm{b5} \\
\text { (deepened) }\end{array}$ & 786 & $736-786$ & $\left\{3.3^{\prime \prime}\right\}$ & $\begin{array}{l}\text { Appendix A4; Technical Memorandum } \\
\text { (Mercer, 1996) }\end{array}$ & $\begin{array}{l}\text { Drilled to conduct tracer tests to define the nature of } \\
\text { solute transport in the Culebra Dolomite \& provide } \\
\text { quantitative estimates of transport parameters } \\
\text { needed for Performance Assessment calculations. } \\
\text { Geologic \& geophysical data were also obtained. } \\
\text { Hole bottomed in lower member of Rustler. } \\
\text { Formation. }\end{array}$ \\
\hline$H-19 b 6$ & 736 & $\begin{array}{l}\text { Not } \\
\text { Cored }\end{array}$ & & $\begin{array}{l}\text { Appendix A4; Technical Memorandum } \\
\text { (Mercer, 1996) }\end{array}$ & $\begin{array}{l}\text { Drilled to conduct tracer tests to define the nature of } \\
\text { solute transport in the Culebra Dolomite \& provide } \\
\text { quantitative estimates of transport parameters } \\
\text { needed for Performance Assessment calculations. } \\
\text { Geologic \& geophysical data were also obtained. } \\
\text { Hole bottomed in Tamarisk member of Rustler } \\
\text { Formation. }\end{array}$ \\
\hline $\begin{array}{c}\mathrm{H}-19 \mathrm{~b} 6 \\
\text { (deepened) }\end{array}$ & 788 & $737-788$ & $\left\{3.3^{\prime \prime}\right\}$ & $\begin{array}{l}\text { Appendix A4; Technical Memorandum } \\
\text { (Mercer, 1996) }\end{array}$ & $\begin{array}{l}\text { Drilled to conduct tracer tests to define the nature of } \\
\text { solute transport in the Culebra Dolomite \& provide } \\
\text { quantitative estimates of transport parameters } \\
\text { needed for Performance Assessment calculations. } \\
\text { Geologic \& geophysical data were also obtained. } \\
\text { Hole bottomed in lower member of Rustler } \\
\text { Formation. }\end{array}$ \\
\hline $\mathrm{H}-19 \mathrm{~b} 7$ & 785 & $\begin{array}{l}679-735 \\
736-783\end{array}$ & $\begin{array}{l}\left\{3.3^{\prime \prime}\right\} \\
\left\{3.3^{\prime \prime}\right\}\end{array}$ & $\begin{array}{l}\text { Appendix A4; Technical Memorandum } \\
\text { (Mercer, 1996) }\end{array}$ & $\begin{array}{l}\text { Drilled to conduct tracer tests to define the nature of } \\
\text { solute transport in the Culebra Dolomite \& provide } \\
\text { quantitative estimates of transport parameters } \\
\text { needed for Performance Assessment calculations. } \\
\text { Geologic \& geophysical data were also obtained. } \\
\text { Hole bottomed in lower member of Rustler } \\
\text { Formation. }\end{array}$ \\
\hline WQSP-1 & 737 & $696-737$ & $\left\{4.0^{\prime \prime}\right\}$ & $\begin{array}{l}\text { DOEMMPP-95-2154 (U.S. Department } \\
\text { of Energy, 1995b) }\end{array}$ & $\begin{array}{l}\text { Drilled by Westinghouse to study the chemical } \\
\text { characteristics (TDS, volatile organics, and metal } \\
\text { concentrations) of groundwater in the Culebra } \\
\text { Dolomite. Well was built (without iron-casing) to } \\
\text { minimize well casing effects on brine chemistry. }\end{array}$ \\
\hline WQSP-2 & 846 & $800-846$ & $\left\{4.0^{\prime \prime}\right\}$ & $\begin{array}{l}\text { DOENWIPP-95-2154 (U.S. Department } \\
\text { of Energy, 1995b) }\end{array}$ & $\begin{array}{l}\text { Drilled by Westinghouse to study the chemical } \\
\text { characteristics (TDS, volatile organics, and metal } \\
\text { concentrations) of groundwater in the Culebra } \\
\text { Dolomite. Well was built (without iron-casing) to } \\
\text { minimize well casing effects on brine chemistry. }\end{array}$ \\
\hline WQSP-3 & 879 & $833-879$ & $\left\{4.0^{\prime \prime}\right\}$ & $\begin{array}{l}\text { DOEMMPP-95-2154 (U.S. Department } \\
\text { of Energy, 1995b) }\end{array}$ & $\begin{array}{l}\text { Drilled by Westinghouse to study the chemical } \\
\text { characteristics (TDS, volatile organics, and metal } \\
\text { concentrations) of groundwater in the Culebra } \\
\text { Dolomite. Well was built (without iron-casing) to } \\
\text { minimize well casing effects on brine chemistry. }\end{array}$ \\
\hline
\end{tabular}


Table 1. DOE-Sponsored Surface Boreholes at the Waste Isolation Pilot Plant (continued)

\begin{tabular}{|c|c|c|c|c|c|c|c|c|}
\hline ID & $\begin{array}{c}\text { Drilling } \\
\text { Start/End } \\
\text { Date }\end{array}$ & Purpose & Status & $\begin{array}{l}\text { Township/ } \\
\text { Range/ } \\
\text { Section }\end{array}$ & $\begin{array}{c}\text { Section } X \& Y \\
\text { Coordinates }\end{array}$ & $\begin{array}{l}\text { State- } \\
\text { Plane } \\
\text { Coord. } \\
X \text { (ft) }\end{array}$ & $\begin{array}{c}\text { State- } \\
\text { Plane } \\
\text { Coord. } \\
Y(\mathrm{ft})\end{array}$ & $\begin{array}{c}\text { Surface } \\
\text { Elevation } \\
\text { (ft) }\end{array}$ \\
\hline WQSP-4 & $\begin{array}{l}10 / 05 / 94 \\
10 / 07 / 94\end{array}$ & HYD & $\begin{array}{l}\text { Monitoring water } \\
\text { quality. }\end{array}$ & T22S, R31E, S28 & $1618 \mathrm{FSL}, 2181 \mathrm{FEL}$ & 670,644 & 494,986 & 3430 \\
\hline WQSP-5 & $\begin{array}{l}10 / 12 / 94 \\
10 / 13 / 94\end{array}$ & HYD & $\begin{array}{l}\text { Monitoring water } \\
\text { quality. }\end{array}$ & T22S, R31E, S29 & 304 FSL, 371 FEL & 667,163 & 493,665 & 3382 \\
\hline WQSP-6 & $\begin{array}{l}09 / 22 / 94 \\
09 / 30 / 94\end{array}$ & HYD & $\begin{array}{l}\text { Monitoring water } \\
\text { quality. }\end{array}$ & T22S, R31E, S29 & $1616 \mathrm{FSL}, 1416 \mathrm{FWL}$ & 663,678 & 494,949 & 3362 \\
\hline WQSP-6a & $\begin{array}{l}10 / 28 / 94 \\
10 / 31 / 94\end{array}$ & HYD & $\begin{array}{l}\text { Monitoring water } \\
\text { quality. }\end{array}$ & T22S, R31E, S29 & $1643 \mathrm{FSL}, 1350 \mathrm{FWL}$ & 663,612 & 494,976 & 3361 \\
\hline \multicolumn{9}{|c|}{ Resource Evaluation } \\
\hline $\begin{array}{c}\text { CB-1 } \\
\text { (CABIN BABY } \\
\text { FED. NO. 1) }\end{array}$ & $\begin{array}{l}05 / 31 / 74= \\
02 / 08 / 75\end{array}$ & $\begin{array}{l}\text { HYDRO- } \\
\text { CARBON }\end{array}$ & Dry petroleum hole. & T23S, R31E, S5 & $1980 \mathrm{FNL}, 1980 \mathrm{FEL}$ & 665,559 & 486,111 & 3320 \\
\hline $\begin{array}{c}\text { CB-1 } \\
\text { (CABIN BABY } \\
\text { FED. NO. 1) } \\
\text { (deepened) }\end{array}$ & $\begin{array}{l}08 / 12 / 83 \\
08 / 28 / 83\end{array}$ & HYD & $\begin{array}{l}\text { Used as a } \\
\text { hydrologic } \\
\text { monitoring well. }\end{array}$ & T23S, R31E, S5 & $1980 \mathrm{FNL}, 1980 \mathrm{FEL}$ & 665,559 & 486,111 & 3320 \\
\hline D-268 & $\begin{array}{c}1984 \\
\text { (specific dates } \\
\text { unknown) }\end{array}$ & POTHYD & $\begin{array}{l}\text { Plugged back to } \\
493 \mathrm{ft} \text {. Used by } \\
\text { Sandia as a } \\
\text { hydrologic } \\
\text { monitoring hole. }\end{array}$ & T22S, R30E, S35 & 720 FSL, $763 \mathrm{FEL}$ & 650,843 & 488,920 & 3279 \\
\hline P-1 & $\begin{array}{l}08 / 23 / 76 \\
09 / 02 / 76\end{array}$ & POT & $\begin{array}{l}\text { Casing pulled and } \\
\text { plugged to surface. } \\
\text { Abandoned on } \\
09 / 02 / 76 \text {. }\end{array}$ & T22S, R31E, S29 & 327 FSL, 551 FWL & 662,804 & 493,651 & 3345 \\
\hline P-2 & $\begin{array}{l}08 / 25 / 76 \\
09 / 03 / 76\end{array}$ & POT & $\begin{array}{l}\text { Casing pulled, } \\
\text { plugged to surface. } \\
\text { Abandoned on } \\
09 / 03 / 76 .\end{array}$ & T22S, R31E, S28 & $125 \mathrm{FNL}, 172 \mathrm{FEL}$ & 672,609 & 498,536 & 3478 \\
\hline P-3 & $\begin{array}{l}08 / 26 / 76 \\
09 / 08 / 76\end{array}$ & POT & $\begin{array}{l}\text { Casing pulled, } \\
\text { plugged to surface. } \\
\text { Abandoned on } \\
09 / 08 / 76 \text {. }\end{array}$ & T22S, R31E, S20 & $103 \mathrm{FSL}, 3122 \mathrm{FEL}$ & 664,351 & 498,747 & 3382 \\
\hline
\end{tabular}


Table 1. DOE-Sponsored Surface Boreholes at the Waste Isolation Pilot Plant (continued)

\begin{tabular}{|c|c|c|c|c|c|}
\hline ID & $\begin{array}{c}\text { Total } \\
\text { Depth }(\mathrm{ft})\end{array}$ & $\begin{array}{l}\text { Depth of } \\
\text { Cored } \\
\text { Interval(s) } \\
\text { (ft) }\end{array}$ & 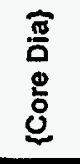 & Reference(s) & Comments \\
\hline WQSP-4 & 800 & $740-798$ & $\left\{4.0^{\prime \prime}\right\}$ & $\begin{array}{l}\text { DOEMMPP-95-2154 (U.S. Department } \\
\text { of Energy, 1995b) }\end{array}$ & $\begin{array}{l}\text { Drilled by Westinghouse to study the chemical } \\
\text { characteristics (TDS, voiatile organics, and metal } \\
\text { concentrations) of groundwater in the Culebra } \\
\text { Dolomite. Well was built (without iron-casing) to } \\
\text { minimize well casing effects on brine chemistry. }\end{array}$ \\
\hline WQSP-5 & 681 & $648-676$ & $\left\{4.0^{\prime \prime}\right\}$ & $\begin{array}{l}\text { DOEMMPP-95-2154 (U.S. Department } \\
\text { of Energy, 1995b) }\end{array}$ & $\begin{array}{l}\text { Drilled by Westinghouse to study the chemical } \\
\text { characteristics (TDS, volatile organics, and metal } \\
\text { concentrations) of groundwater in the Culebra } \\
\text { Dolomite. Well was built (without iron-casing) to } \\
\text { minimize well casing effects on brine chemistry. }\end{array}$ \\
\hline WQSP-6 & 617 & $568-617$ & $\left\{4.0^{\prime \prime}\right\}$ & $\begin{array}{l}\text { DOEMIPP-95-2154 (U.S. Department } \\
\text { of Energy, 1995b) }\end{array}$ & $\begin{array}{l}\text { Drilled by Westinghouse to study the chemical } \\
\text { characteristics (TDS, volatile organics, and metal } \\
\text { concentrations) of groundwater in the Culebra } \\
\text { Dolomite. Well was built (without iron-casing) to } \\
\text { minimize well casing effects on brine chemistry. }\end{array}$ \\
\hline WQSP-6a & 225 & $160-220$ & $\left\{4.0^{0}\right\}$ & $\begin{array}{l}\text { DOEMMPP-95-2154 (U.S. Department } \\
\text { of Energy, 1995b) }\end{array}$ & $\begin{array}{l}\text { Drilled by Westinghouse to study the chemical } \\
\text { characteristics (TDS, volatile organics, and metal } \\
\text { concentrations) of groundwater in the Dewey Lake } \\
\text { Red Beds. Well was built (without iron-casing) to } \\
\text { minimize well casing effects on brine chemistry. }\end{array}$ \\
\hline \multicolumn{6}{|c|}{ Resource Evaluation } \\
\hline $\begin{array}{c}\text { CB-1 } \\
\text { (CABIN BABY } \\
\text { FED. NO. 1) }\end{array}$ & $4151^{*}$ & $\begin{array}{l}\text { Not } \\
\text { Cored }\end{array}$ & & Private Oil Company & $\begin{array}{l}\text { Drilled initially as a hydrocarbon well, control was } \\
\text { turned over to the DOE after it was found to be a "dry } \\
\text { hole." }\end{array}$ \\
\hline $\begin{array}{l}\text { CB-1 } \\
\text { (CABIN BABY } \\
\text { FED. NO. 1) } \\
\text { (deepened) }\end{array}$ & $4291^{*}$ & $4151-4291$ & $\left\{4.0^{\prime \prime}\right\}$ & $\begin{array}{l}\text { WTSD-TME-020 (Beauheim et al., } \\
\text { 1983), SAND87-7125 (Stensrud et al., } \\
\text { 1987) }\end{array}$ & $\begin{array}{l}\text { Re-entered in } 1983 \text { to provide data on hydrologic } \\
\text { properties (including hydrostatic head) and provide } \\
\text { fluid samples from selected permeable zones in Bell } \\
\text { Canyon Formation and further define upper Bell } \\
\text { Canyon stratigraphy. }\end{array}$ \\
\hline D-268 & 1411 & $528-1411$ & $\{?\}$ & SAND89-7056 (Stensrud et al., 1990) & $\begin{array}{l}\text { Drilled by Duval Mining Co. as a potash exploration } \\
\text { well. Sandia later developed the well } \\
\text { (4/12/88) to provide test data to estimate the } \\
\text { Culebra's hydraulic properties and evaluate } \\
\text { hydraulic responses. Slug injection \& water quality } \\
\text { tests conducted. }\end{array}$ \\
\hline P-1 & 1591 & $1200-1591$ & $\{2.2 "\}$ & $\begin{array}{l}\text { SANDT7-1217 (Griswold, 1977b), } \\
\text { USGS OFR 78-592 (Jones, 1978) }\end{array}$ & $\begin{array}{l}\text { Evaluations in } 1976 \text { indicated low-grade langbeinite } \\
\text { mineralization in ore zone } 5 \text {. Hole bottomed } \\
\text { immediately below McNutt potash zone of Salado } \\
\text { Formation. }\end{array}$ \\
\hline$P-2$ & 1895 & $1500-1895$ & $\left\{2.2^{2}\right\}$ & $\begin{array}{l}\text { SAND77-1217 (Griswold, 1977b), } \\
\text { USGS OFR 78-592 (Jones, 1978) }\end{array}$ & $\begin{array}{l}\text { Evaluations in } 1976 \text { indicated lease-grade langbeinite } \\
\text { mineralization in ore zone } 10 \text {. Low-grade langbeinite } \\
\text { mineralization in zones } 4 \& 2 \text {. Hole bottomed } \\
\text { immediately below McNutt potash zone of Salado } \\
\text { Formation. }\end{array}$ \\
\hline P-3 & 1676 & $1300-1676$ & $\left\{2.2^{2}\right\}$ & $\begin{array}{l}\text { SAND77-1217 (Griswold, 1977b), } \\
\text { USGS OFR 78-592 (Jones, 1978) }\end{array}$ & $\begin{array}{l}\text { Evaluations in } 1976 \text { indicated low-grade langbeinite } \\
\text { mineralization in ore zone } 4 \text {. Hole bottomed } \\
\text { immediately below McNutt potash zone of Salado } \\
\text { Formation. }\end{array}$ \\
\hline
\end{tabular}


Table 1. DOE-Sponsored Surface Boreholes at the Waste Isolation Pilot Plant (continued)

\begin{tabular}{|c|c|c|c|c|c|c|c|c|}
\hline ID & $\begin{array}{c}\text { Drilling } \\
\text { Start/End } \\
\text { Date }\end{array}$ & Purpose & Status & $\begin{array}{l}\text { Township/ } \\
\text { Range/ } \\
\text { Section }\end{array}$ & $\begin{array}{c}\text { Section } X \& Y \\
\text { Coordinates }\end{array}$ & $\begin{array}{l}\text { State- } \\
\text { Plane } \\
\text { Coord. } \\
\text { X (ft) }\end{array}$ & $\begin{array}{l}\text { State- } \\
\text { Plane } \\
\text { Coord. } \\
Y(f t)\end{array}$ & $\begin{array}{c}\text { Surface } \\
\text { Elevation } \\
\text { (ft) }\end{array}$ \\
\hline$P-4$ & $\begin{array}{l}08 / 27 / 76- \\
09 / 07 / 76\end{array}$ & POT & $\begin{array}{l}\text { Casing pulled, } \\
\text { plugged to surface. } \\
\text { Abandoned on } \\
09 / 07 / 76 \text {. }\end{array}$ & T22S, R31E, S28 & 146 FSL, 1487 FEL & 671,327 & 493,521 & 3441 \\
\hline P-5 & $\begin{array}{l}09 / 10 / 76- \\
09 / 22 / 76\end{array}$ & POT & $\begin{array}{l}\text { Casing pulled, } \\
\text { plugged to surface. } \\
\text { Abandoned on } \\
09 / 22276 \text {. }\end{array}$ & T22S, R31E, S17 & $202 \mathrm{FSL}, 165 \mathrm{FEL}$ & 667,286 & 504,120 & 3472 \\
\hline P-6 & $\begin{array}{c}09 / 03 / 76- \\
09 / 17 / 76\end{array}$ & POT & $\begin{array}{l}\text { Casing pulled, } \\
\text { plugged to surface. } \\
\text { Plugged and } \\
\text { abandoned on } \\
09 / 17 / 76 .\end{array}$ & T22S, R31E, S30 & $2767 \mathrm{FSL}, 199 \mathrm{FWL}$ & 657,144 & 496,090 & 3354 \\
\hline P-7 & $\begin{array}{l}09 / 04 / 76- \\
09 / 22 / 76\end{array}$ & POT & $\begin{array}{l}\text { Casing pulled, } \\
\text { plugged to surface. } \\
\text { Abandoned on } \\
09 / 22 / 76 \text {. }\end{array}$ & T23S, R31E, S5 & $513 \mathrm{FNL}, 396 \mathrm{FWL}$ & 662,669 & 487,090 & 3332 \\
\hline P-8 & $\begin{array}{l}09 / 08 / 76- \\
09 / 15 / 76\end{array}$ & POT & $\begin{array}{l}\text { Casing pulled, } \\
\text { plugged to surface. } \\
\text { Abandoned on } \\
09 / 15 / 76 \text {. }\end{array}$ & T23S, R31E, S4 & 642 FNL, 96 FWL & 667,664 & 487,471 & 3336 \\
\hline P-9 & $\begin{array}{l}09 / 16 / 76 \\
09 / 26 / 76\end{array}$ & POT & $\begin{array}{l}\text { Casing pulled, } \\
\text { plugged to surface. } \\
\text { Plugged and } \\
\text { abandoned on } \\
09 / 26 / 76 \text {. }\end{array}$ & T22S, R31E, S33 & $1493 \mathrm{FSL}, 143 \mathrm{FEL}$ & 672,678 & 489,599 & 3409 \\
\hline P-10 & $\begin{array}{c}09 / 24 / 76- \\
10 / 17 / 76\end{array}$ & POT & $\begin{array}{l}\text { Casing pulled, } \\
\text { plugged to surface. } \\
\text { Abandoned on } \\
10 / 17 / 76 .\end{array}$ & T22S, R31E, S26 & $2315 \mathrm{FNL}, 339 \mathrm{FWL}$ & 678,410 & 496,383 & 3508 \\
\hline P-11 & $\begin{array}{c}09 / 24 / 76- \\
10 / 16 / 76\end{array}$ & POT & $\begin{array}{l}\text { Casing pulled, } \\
\text { plugged to surface. } \\
\text { Abandoned on } \\
10 / 18 / 76 \text {. }\end{array}$ & T22S, R31E, S23 & $175 \mathrm{FNL}, 177 \mathrm{FWL}$ & 678,217 & 503,781 & 3506 \\
\hline P-12 & $\begin{array}{c}09 / 17 / 76- \\
10 / 20 / 76\end{array}$ & POT & $\begin{array}{l}\text { Casing pulled, } \\
\text { plugged to surface. } \\
\text { Abandoned on } \\
10 / 23 / 76 \text {. }\end{array}$ & T22S, R30E, S24 & $167 \mathrm{FNL}, 195 \mathrm{FEL}$ & 656,692 & 503,896 & 3376 \\
\hline P-13 & $\begin{array}{l}09 / 17 / 76- \\
09 / 23 / 76\end{array}$ & POT & $\begin{array}{l}\text { Casing pulled, } \\
\text { plugged to surface. } \\
\text { Abandoned on } \\
09 / 24 / 76 \text {. }\end{array}$ & T22S, R31E, S18 & $125 \mathrm{FNL}, 116 \mathrm{FWL}$ & 656,973 & 509,039 & 3345 \\
\hline
\end{tabular}


Table 1. DOE-Sponsored Surface Boreholes at the Waste Isolation Pilot Plant (continued)

\begin{tabular}{|c|c|c|c|c|c|}
\hline ID & $\mid \begin{array}{c}\text { Total } \\
\text { Depth }(\mathrm{ft})\end{array}$ & $\begin{array}{l}\text { Depth of } \\
\text { Cored } \\
\text { Interval(s) } \\
\text { (ft) }\end{array}$ & 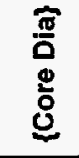 & Reference(s) & Comments \\
\hline$P-4$ & 1857 & $1460-1857$ & $\left\{2.2^{\prime \prime}\right\}$ & $\begin{array}{l}\text { SAND77-1217 (Griswold, 1977b), } \\
\text { USGS OFR 78-592 (Jones, 1978) }\end{array}$ & $\begin{array}{l}\text { Evaluations in } 1976 \text { indicated lease-grade sylvite in } \\
\text { ore zone } 10 \text {. Hole bottomed immediately below } \\
\text { McNutt potash zone of Salado Formation. }\end{array}$ \\
\hline P-5. & 1830 & $1510-1830$ & $\left\{2.2^{\prime \prime}\right\}$ & $\begin{array}{l}\text { SAND77-1217 (Griswold, 1977b), } \\
\text { USGS OFR 78-592 (Jones, 1978) }\end{array}$ & $\begin{array}{l}\text { Evaluations in } 1976 \text { indicated lease-grade sylvite in } \\
\text { ore zone } 10 . \text { Hole bottomed immediately below } \\
\text { McNutt potash zone of Salado Formation. }\end{array}$ \\
\hline$P-6$ & 1573 & $1150-1573$ & $\left\{2.2^{\prime \prime}\right\}$ & $\begin{array}{l}\text { SAND77-1217 (Griswold, 1977b), } \\
\text { USGS OFR 78-592 (Jones, 1978) }\end{array}$ & $\begin{array}{l}\text { Evaluations in } 1976 \text { indicated marginal langbeinite in } \\
\text { ore zones } 4 \& 2 \text {. Hole bottomed immediately below } \\
\text { McNutt potash zone of Salado Formation. }\end{array}$ \\
\hline P-7 & 1574 & $1264-1574$ & $\left\{2.2^{\prime \prime}\right\}$ & $\begin{array}{l}\text { SANO77-1217 (Griswold, 1977b), } \\
\text { USGS OFR 78-592 (Jones, 1978) }\end{array}$ & $\begin{array}{l}\text { Evaluations in } 1976 \text { indicated low-grade langbeinite in } \\
\text { ore zone 4. Hole bottomed immediately below } \\
\text { McNutt potash zone of Salado Formation. }\end{array}$ \\
\hline P-8 & 1660 & $1240-1660$ & $\left\{2.2^{\prime \prime}\right\}$ & $\begin{array}{l}\text { SAND77-1217 (Griswold, 1977b), } \\
\text { USGS OFR 78-592 (Jones, 1978) }\end{array}$ & $\begin{array}{l}\text { Evaluations in } 1976 \text { indicated lease-grade mixed ore } \\
\text { in ore zone } 10 \text {. Hole bottomed immediately below } \\
\text { McNutt potash zone of Salado Formation. }\end{array}$ \\
\hline P-9 & 1796 & $1410-1796$ & $\left\{2.2^{\prime \prime}\right\}$ & $\begin{array}{l}\text { SAND77-1217 (Griswold, 1977b), } \\
\text { USGS OFR 78-592 (Jones, 1978), } \\
\text { Sanchez and McCasland (1994) }\end{array}$ & $\begin{array}{l}\text { Evaluations in } 1976 \text { indicated lease-grade mixed ores } \\
\text { in ore zone } 10 . \text { Borehole log suggested perched } \\
\text { water-bearing zone in the Dewey Lake Red Beds } \\
\text { Formation. Hole boltomed immediately below McNutt } \\
\text { potash zone of Salado Formation. }\end{array}$ \\
\hline P-10 & 2009 & $1620-2009$ & $\left\{2.2^{\prime \prime}\right\}$ & $\begin{array}{l}\text { SAND77-1217 (Griswold, 1977b), } \\
\text { USGS OFR 78-592 (Jones, 1978) }\end{array}$ & $\begin{array}{l}\text { Evaluations in } 1976 \text { indicated low-grade sylvite in ore } \\
\text { zone 11. Hole bottomed immediately below McNutt } \\
\text { potash zone of Salado Formation. }\end{array}$ \\
\hline P-11 & 1940 & 1580-1940 & $\left\{2.2^{\prime \prime}\right\}$ & $\begin{array}{l}\text { SAND77-1217 (Griswold, 1977b), } \\
\text { USGS OFR 78-592 (Jones, 1978) }\end{array}$ & $\begin{array}{l}\text { Evaluations in } 1976 \text { indicated lease-grade langbeinite } \\
\text { in ore zone } 2 \text {. Low grade mineralization in zones } 11, \\
10, \& 4 \text {. Lease grade sylvite in ore zone } 9 \text {. Hole } \\
\text { bottomed immediately below McNutt potash zone of } \\
\text { Salado Formation. }\end{array}$ \\
\hline P-12 & 1598 & 1280-1598 & $\left\{2.2^{\prime \prime}\right\}$ & $\begin{array}{l}\text { SAND77-1217 (Griswold, 1977b), } \\
\text { USGS OFR 78-592 (Jones, 1978) }\end{array}$ & $\begin{array}{l}\text { Evaluations in } 1976 \text { indicated lease-grade sylvite in } \\
\text { ore zone } 10 \text {. Mixed ore in zone } 4 \text {. Hole bottomed } \\
\text { immediately below McNutt potash zone of Salado } \\
\text { Formation. }\end{array}$ \\
\hline P-13 & 1576 & $1220-1576$ & $\left\{2.2^{\prime \prime}\right\}$ & $\begin{array}{l}\text { SAND77-1217 (Griswold, 1977b), } \\
\text { USGS OFR 78-592 (Jones, 1978) }\end{array}$ & $\begin{array}{l}\text { Evaluations in } 1976 \text { indicated lease-grade sylvite in } \\
\text { ore zone } 10 \text {. Low grade mineralization in zones } 9,8 \text {, } \\
4, \& 3 \text {. Hole bottomed immediately below McNutt } \\
\text { potash zone of Salado Formation. }\end{array}$ \\
\hline
\end{tabular}


Table 1. DOE-Sponsored Surface Boreholes at the Waste Isolation Pilot Plant (continued)

\begin{tabular}{|c|c|c|c|c|c|c|c|c|}
\hline ID & $\begin{array}{l}\text { Drilling } \\
\text { Start/End } \\
\text { Date }\end{array}$ & Purpose & Status & $\begin{array}{l}\text { Township/ } \\
\text { Range/ } \\
\text { Section }\end{array}$ & $\begin{array}{l}\text { Section } X \& Y \\
\text { Coordinates }\end{array}$ & $\begin{array}{l}\text { State- } \\
\text { Plane } \\
\text { Coord. } \\
\text { X (ft) }\end{array}$ & $\begin{array}{c}\text { State- } \\
\text { Plane } \\
\text { Coord. } \\
Y \text { (ft) }\end{array}$ & $\begin{array}{c}\text { Surface } \\
\text { Elevation } \\
\text { (ft) }\end{array}$ \\
\hline P-14 & $\begin{array}{l}09 / 24 / 76 \\
10 / 03 / 76\end{array}$ & POTHYD & $\begin{array}{l}\text { Plugged from } 1545 \\
\text { to } 759 \mathrm{ft} \text {. Used as } \\
\text { hydrologic } \\
\text { monitoring well. } \\
\text { Cased from 0-775 } \\
\text { ft. }\end{array}$ & T22S, R30E, S24 & 312 FSL, 613 FWL & 652,159 & 499,082 & 3358 \\
\hline P-15 & $\begin{array}{l}10 / 04 / 76 \\
10 / 14 / 76\end{array}$ & POTHYD & $\begin{array}{l}\text { Plugged from } 1465 \\
620 \mathrm{ft} \text {. Used as } \\
\text { hydrologic } \\
\text { monitoring weil. } \\
\text { Cased from } 0-635 \\
\text { ft. }\end{array}$ & T22S, R31E, S31 & 411 FSL, $192 \mathrm{FWL}$ & 657,148 & 488,426 & 3310 \\
\hline P-16 & $\begin{array}{c}09 / 27 / 76- \\
10 / 05 / 76\end{array}$ & POT & $\begin{array}{l}\text { Casing pulled, } \\
\text { plugged to surface. } \\
\text { Abandoned on } \\
10 / 06 / 76 .\end{array}$ & T23S, R31E, S5 & 951 FSL, 1629 FWL & 663,914 & 483,737 & 3323 \\
\hline P-17 & $\begin{array}{c}10 / 18 / 76- \\
10 / 26 / 76\end{array}$ & POTHYD & $\begin{array}{l}\text { Hole plugged from } \\
1660-731 \mathrm{ft} \text {. Used } \\
\text { as hydrologic } \\
\text { monitoring well. } \\
\text { Hole cased from } 0- \\
751 \mathrm{ft}\end{array}$ & T23S, R31E, S4 & 1351 FSL, 395 FWL & 667,955 & 484,185 & 3340 \\
\hline P-18 & $\begin{array}{l}10 / 19 / 76- \\
11 / 05 / 76\end{array}$ & POTHYD & $\begin{array}{l}\text { Hole plugged from } \\
1998-1125 \text {. Used } \\
\text { as hydrologic } \\
\text { monitoring well. } \\
\text { Hole cased from } 0- \\
1138 \mathrm{ft} \text {. }\end{array}$ & T22S, R31E, S26 & 134 FSL, $797 \mathrm{FEL}$ & 682,589 & 493,556 & 3478 \\
\hline P-19 & $\begin{array}{c}10 / 19 / 76- \\
11 / 04 / 76\end{array}$ & РОт & $\begin{array}{l}\text { Casing pulled, } \\
\text { plugged to surface. } \\
\text { Abandoned on } \\
11 / 04 / 76\end{array}$ & T22S, R31E, S23 & $1652 \mathrm{FSL}, 2330 \mathrm{FWL}$ & 680,377 & 500,362 & 3546 \\
\hline P-20 & $\begin{array}{l}10 / 06 / 76- \\
10 / 14 / 76\end{array}$ & РOT & \begin{tabular}{|l|} 
Casing pulled, \\
plugged to surface. \\
Abandoned on \\
$10 / 15 / 76$.
\end{tabular} & T22S, R31E, S14 & $794 \mathrm{FSL}, 103 \mathrm{FEL}$ & 683,197 & 504,767 & 3553 \\
\hline P-21 & $\begin{array}{l}10 / 15 / 76- \\
10 / 26 / 76\end{array}$ & POT & $\begin{array}{l}\text { Casing pulled, } \\
\text { plugged to surface. } \\
\text { Abandoned on } \\
10 / 27 / 76 \text {. }\end{array}$ & T22S, R31E, S15 & $852 \mathrm{FNL}, 150 \mathrm{FEL}$ & 677,862 & 508,351 & 3510 \\
\hline
\end{tabular}


Table 1. DOE-Sponsored Surface Boreholes at the Waste Isolation Pilot Plant (continued)

\begin{tabular}{|c|c|c|c|c|c|}
\hline ID & $\begin{array}{c}\text { Total } \\
\text { Depth }(\mathrm{ft})\end{array}$ & $\begin{array}{l}\text { Depth of } \\
\text { Cored } \\
\text { Interval(s) } \\
\text { (ft) }\end{array}$ & 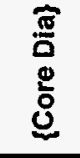 & Reference(s) & Comments \\
\hline P-14 & 1545 & $1188-1545$ & $\left\{2.2^{\prime \prime}\right\}$ & $\begin{array}{l}\text { SAND77-1217 (Griswold, 19776), } \\
\text { USGS OFR 78-592 (Jones, 1978), } \\
\text { SAND89-7056 (Stensrud et al., 1990), } \\
\text { USGS-WRI 79-98 (Mercer and Orr, } \\
\text { 1979) }\end{array}$ & $\begin{array}{l}\text { Evaluations in } 1976 \text { indicated lease-grade sylvite in } \\
\text { ore zone } 10 \text {. Casing perforated for hydrologic } \\
\text { monitoring in the Culebra Dolomite \& Rustler/Salado } \\
\text { contact. Hole bottomed immediately below McNutt } \\
\text { potash zone of Salado Formation. }\end{array}$ \\
\hline$P-15$ & 1465 & $\begin{array}{c}515-600 \\
1038-1465\end{array}$ & $\begin{array}{l}\left\{2.2^{\prime \prime}\right\} \\
\left\{2.2^{\prime \prime}\right\}\end{array}$ & $\begin{array}{l}\text { SAND77-1217 (Griswold, 1977b), } \\
\text { USGS OFR 78-592 (Jones, 1978), } \\
\text { SAND87-7166 (Stensrud et al., 1988a), } \\
\text { Sanchez and McCasland (1994), } \\
\text { USGS-WRI 79-98 (Mercer and Orr, } \\
\text { 1979) }\end{array}$ & $\begin{array}{l}\text { Evaluations in } 1976 \text { indicated low-grade langbeinite in } \\
\text { ore zones } 4 \text { \& } 2 \text {. Casing perforated for hydrologic } \\
\text { monitoring in the Culebra Dolomite \& Rustler/Salado } \\
\text { contact. Hole bottomed immediately below McNutt } \\
\text { potash zone of Salado Formation. }\end{array}$ \\
\hline P-16 & 1585 & $1190-1585$ & $\left\{2.2^{\prime \prime}\right\}$ & $\begin{array}{l}\text { SAND77-1217 (Griswold, 1977b), } \\
\text { USGS OFR 78-592 (Jones, 1978) }\end{array}$ & $\begin{array}{l}\text { Evaluations in } 1976 \text { indicated lease-grade langbeinite } \\
\text { in ore zone } 4 \text {. Hole bottomed immediately below } \\
\text { McNutt potash zone of Salado Formation. }\end{array}$ \\
\hline$P-17$ & 1660 & $1220-1660$ & $\left\{2.2^{\prime \prime}\right\}$ & $\begin{array}{l}\text { SAND77-1217 (Griswold, 1977b), } \\
\text { USGS OFR 78-592 (Jones, 1978), } \\
\text { Sanchez and McCasland (1994), } \\
\text { USGS WRI 79-98 (Mercer and Orr, } \\
\text { 1979), SAND87-7125 (Stensrud et al., } \\
\text { 1987) }\end{array}$ & $\begin{array}{l}\text { Evaluations in } 1976 \text { indicated lease-grade langbeinite } \\
\text { in ore zone } 4 . \text { Low grade langbeinite in zone } 2 . \\
\text { Casing perforated for monitoring the Culebra } \\
\text { Dolomite \& Rustler/Salado contact. Hole bottomed } \\
\text { immediately below McNutt potash zone of Salado } \\
\text { Formation. }\end{array}$ \\
\hline P-18 & 1998 & $1630-1998$ & $\left\{2.2^{\prime}\right\}$ & $\begin{array}{l}\text { SAND77-1217 (Griswold, 1977b), } \\
\text { USGS OFR 78-592 (Jones, 1978), } \\
\text { USGS-WRI 79-98 (Mercer and Orr, } \\
\text { 1979), SAND88-7014 (Stensrud et al., } \\
\text { 1988b) }\end{array}$ & $\begin{array}{l}\text { Evaluations in } 1976 \text { indicated low-grade mixed ores in } \\
\text { ore zone } 10 . \text { Casing perforated for hydrologic } \\
\text { monitoring in the Culebra Dolomite \& Rustler/Salado } \\
\text { contact. Hole bottomed immediately below MCNutt } \\
\text { potash zone of Salado Formation. }\end{array}$ \\
\hline P-19 & 2000 & $1635-2000$ & $\left\{2.2^{\prime \prime}\right\}$ & $\begin{array}{l}\text { SAND77-1217 (Griswold, 1977b), } \\
\text { USGS OFR 78-592 (Jones, 1978) }\end{array}$ & $\begin{array}{l}\text { Evaluations in } 1976 \text { indicated lease-grade langbeinite } \\
\text { in ore zone 10; lower grade langbeinite in zones } 4 \text { \& } \\
\text { 2. Hole bottomed in lower part of McNutt potash zone } \\
\text { of Salado Formation. }\end{array}$ \\
\hline P-20 & 1995 & $1620-1995$ & $\left\{2.2^{\prime \prime}\right\}$ & $\begin{array}{l}\text { SAND77-1217 (Griswold, 1977b), } \\
\text { USGS OFR 78-592 (Jones, 1978) }\end{array}$ & $\begin{array}{l}\text { Evaluations in } 1976 \text { indicated lease-grade sylvite in } \\
\text { ore zone 10; langbeinite in zone 4. Hole bottomed } \\
\text { immediately below McNutt potash zone of Salado } \\
\text { Formation. }\end{array}$ \\
\hline P-21 & 1915 & 1510-1915 & $\left\{2.2^{\prime \prime}\right\}$ & $\begin{array}{l}\text { SAND77-1217 (Griswold, 1977b), } \\
\text { USGS OFR 78-592 (Jones, 1978) }\end{array}$ & $\begin{array}{l}\text { Evaluations in } 1976 \text { indicated lease-grade sylvite in } \\
\text { ore zones } 10 \& 8 \text {. Langbeinite in zone } 4 \text {. Hole } \\
\text { bottomed immediately below MoNutt potash zone of } \\
\text { Salado Formation. }\end{array}$ \\
\hline
\end{tabular}


Table 1. DOE-Sponsored Surface Boreholes at the Waste Isolation Pilot Plant (continued)

\begin{tabular}{|c|c|c|c|c|c|c|c|c|}
\hline ID & $\begin{array}{c}\text { Drilling } \\
\text { Start/End } \\
\text { Date }\end{array}$ & Purpose & Status & $\begin{array}{l}\text { Township/ } \\
\text { Range/ } \\
\text { Section }\end{array}$ & $\begin{array}{l}\text { Section } X \& Y \\
\text { Coordinates }\end{array}$ & $\begin{array}{l}\text { State- } \\
\text { Plane } \\
\text { Coord. } \\
\text { X (ft) }\end{array}$ & $\begin{array}{c}\text { State- } \\
\text { Plane } \\
\text { Coord. } \\
Y(\mathrm{ft})\end{array}$ & $\begin{array}{c}\text { Surface } \\
\text { Elevation } \\
\text { (ft) }\end{array}$ \\
\hline \multicolumn{9}{|c|}{ Shaft Construction } \\
\hline $\begin{array}{c}\text { Salt Handling } \\
\text { (Exploratory) } \\
\text { Shaft }\end{array}$ & $\begin{array}{c}07 / 04 / 81 \\
12 / 20 / 81\end{array}$ & $\begin{array}{l}\text { Designed with } \\
\text { loading pocket } \\
\text { for transport of } \\
\text { mined salt. } \\
\text { Also used for } \\
\text { personnel } \\
\text { transport. }\end{array}$ & Open-In Use & T22S, R31E, S20 & $1070 \mathrm{FSL}, 599 \mathrm{FEL}^{5}$ & 666,895 & 499,687 & 3411 \\
\hline Waste Shaft & $\begin{array}{l}12 / 24 / 81- \\
03 / 10 / 82\end{array}$ & $\begin{array}{l}\text { Transport of } \\
\text { materials and } \\
\text { personnel; } \\
\text { eventual waste } \\
\text { handling shaft } \\
\text { for the facility. }\end{array}$ & Open-In Use & T22S, R31E, S20 & $670 \mathrm{FSL}, 576 \mathrm{FEL}^{5}$ & 666,920 & 499,287 & 3409 \\
\hline Exhaust Shaft & $\begin{array}{l}09 / 22 / 83- \\
02 / 10 / 84\end{array}$ & $\begin{array}{l}\text { Air exhaust } \\
\text { shaft for the } \\
\text { facility. }\end{array}$ & Open-In Use & T22S, R31E, S20 & $668 \mathrm{FSL}, 126 \mathrm{FEL}^{5}$ & 667,370 & 499,287 & 3410 \\
\hline Air Intake Shaft & $\begin{array}{c}12 / 04 / 87= \\
08 / 25 / 88\end{array}$ & $\begin{array}{l}\text { Air intake } \\
\text { source for the } \\
\text { facility. }\end{array}$ & Open-In Use & T22S, R31E, S20 & $1074 \mathrm{FSL}, 1224 \mathrm{FEL}^{5}$ & 666,270 & 499,687 & 3410 \\
\hline
\end{tabular}


Table 1. DOE-Sponsored Surface Boreholes at the Waste Isolation Pilot Plant (continued)

\begin{tabular}{|c|c|c|c|c|c|}
\hline ID & $\begin{array}{c}\text { Total } \\
\text { Depth (ft) }\end{array}$ & $\begin{array}{l}\text { Depth of } \\
\text { Cored } \\
\text { Interval(s) } \\
\text { (ft) }\end{array}$ & $\begin{array}{l}\text { 苗 } \\
\frac{0}{0} \\
0 \\
0\end{array}$ & Reference(s) & Comments \\
\hline \multicolumn{6}{|c|}{ Shaft Construction } \\
\hline $\begin{array}{c}\text { Salt Handling } \\
\text { (Expioratory) } \\
\text { Shaft }\end{array}$ & $\begin{array}{c}2276 \\
\text { (including } \\
\text { sump) }\end{array}$ & $\begin{array}{l}\text { Samples } \\
\text { collected } \\
\text { during mapping } \\
\text { of shaft. }\end{array}$ & & $\begin{array}{l}\text { TME } 3178 \text { (Jarolimek et al., 1983), } \\
\text { Appendix } B^{6} ; \text { As-built WPP Shaft } \\
\text { drawings (No. 09-CS-37-G01 and 09- } \\
\text { CS-37-G02) }\end{array}$ & $\begin{array}{l}\text { Originally called Exploratory Shaft, later named the } \\
\text { Construction \& Salt Handling Shaft, now shortened } \\
\text { to Salt Handling Shaft. Fitted with a } 10 \mathrm{ft} \text { inside } \\
\text { diameter steel liner to a depth of } 845 \mathrm{ft} \text {, just below } \\
\text { the Rustler/Salado contact. Completed in lower unit } \\
\text { of Salado Formation. }\end{array}$ \\
\hline Waste Shaft & $\begin{array}{c}2286 \\
\text { (including } \\
\text { sump) }\end{array}$ & $\begin{array}{l}\text { Samples } \\
\text { collected } \\
\text { during mapping } \\
\text { of shaft. }\end{array}$ & & $\begin{array}{l}\text { WTSD-TME-038 (Holt and Powers, } \\
\text { 1984), Appendix } \mathrm{B}^{6} \text {; As-built WIPP } \\
\text { Shaft drawings (No. 09-CS-31-G01 } \\
\text { and 09-CS-31-G02), SAND87-7166 } \\
\text { (Stensrud et al., 1988a) }\end{array}$ & $\begin{array}{l}\text { Originally drilled to a diameter of } 6 \mathrm{ft} \text { and known as } \\
\text { the Site Preliminary Design Validation (SPDV) } \\
\text { Ventilation Shaft. Enlarged to present } 20 \mathrm{ft} \\
\text { diameter by down slashing in } 1983 \text { to function as } \\
\text { the Waste Handling Shaft (shortened to Waste } \\
\text { Shaft). Lined with concrete to a depth of } 900 \mathrm{ft} \text { and } \\
\text { supported with wire mesh from there to the bottom. } \\
\text { Completed in lower unit of Salado Formation. }\end{array}$ \\
\hline Exhaust Shaft & $\begin{array}{c}2158 \\
\text { (no sump) }\end{array}$ & $\begin{array}{l}\text { Samples } \\
\text { collected } \\
\text { during mapping } \\
\text { of shaft. }\end{array}$ & & $\begin{array}{l}\text { DOEWMPP-86-008 (Holt and Powers, } \\
\text { 1986), Appendix } B^{6} ; \text { As-built WIPP } \\
\text { Shaft drawings (No. 09-CS-35-G01 } \\
\text { and 09-CS-35-G02) }\end{array}$ & $\begin{array}{l}\text { Shaft was up-reamed and down-slashed to a } \\
\text { diameter of } 15 \mathrm{ft} \text {, then lined with concrete to a depth } \\
\text { of } 907 \mathrm{ft} \text {. Walls are supported with steel mesh below } \\
\text { the lining. }\end{array}$ \\
\hline Air Intake Shaft & $\begin{array}{c}2150 \\
\text { (no sump) }\end{array}$ & $\begin{array}{l}\text { Samples } \\
\text { collected } \\
\text { during mapping } \\
\text { of shaft. }\end{array}$ & & $\begin{array}{l}\text { DOEMIPP 90-051 (Holt and Powers, } \\
\text { 1990), SAND89-7056 (Stensrud et al., } \\
\text { 1990), SAND94-1311 (Munson et al., } \\
\text { 1995), Appendix B }{ }^{6} \text {; As-built WIPP } \\
\text { Shaft drawings (No. 09-CS-33-G01 } \\
\text { and 09-CS-33-G02) }\end{array}$ & $\begin{array}{l}\text { Shaft was excavated with a } 20.25 \mathrm{ft} \text { diameter raise- } \\
\text { boring head and lined with concrete to a depth of } 914 \\
\text { ft. Walls are supported intermittently with steel mesh } \\
\text { below the lining. }\end{array}$ \\
\hline
\end{tabular}

Footnotes:

1 State-Plane Coordinates (both X \&Y) for WIPP 14 listed in Table 3-6 of SAND88-1065 (Gonzales, 1989) were in error and have been corrected according to the original survey data reported in SAND82-1783 (Sandia and D'Appolonia, 1982c). In Table 3-6 the coordinates were incorrectly reported as $\mathrm{X}=665,336$ and $\mathrm{Y}=$ 509,260. Correct values are: $X=670,628$ and $Y=509,285$.

2 State-Plane Coordinate X for WIPP 31 listed in Table 3-6 of SAND88-1065 (Gonzales, 1989) is in error and has been corrected based on recalculated data from Appendices $C$ and $D$ of the same report. In Table 3-6 the $X$ coordinate was incorrectly reported as $X=679,527$. Correct value is $X=619,615$.

3 The section number for WIPP 34 listed in Table 3-5 of SAND88-1065 (Gonzales, 1989) is in error and has been corrected based on the Survey Monument "As-Built" information in Appendix C of the same report. The section number was incorrectly reported as Section 8; it is actually Section 9.

4 Appendix A, found in SAND96-1960

${ }^{5}$ Section X \& Y coordinates were calculated from state-plane coordinates using methods consistent with those reported in Gonzales (1989).

${ }^{6}$ Appendix B, found in SAND96-1960 


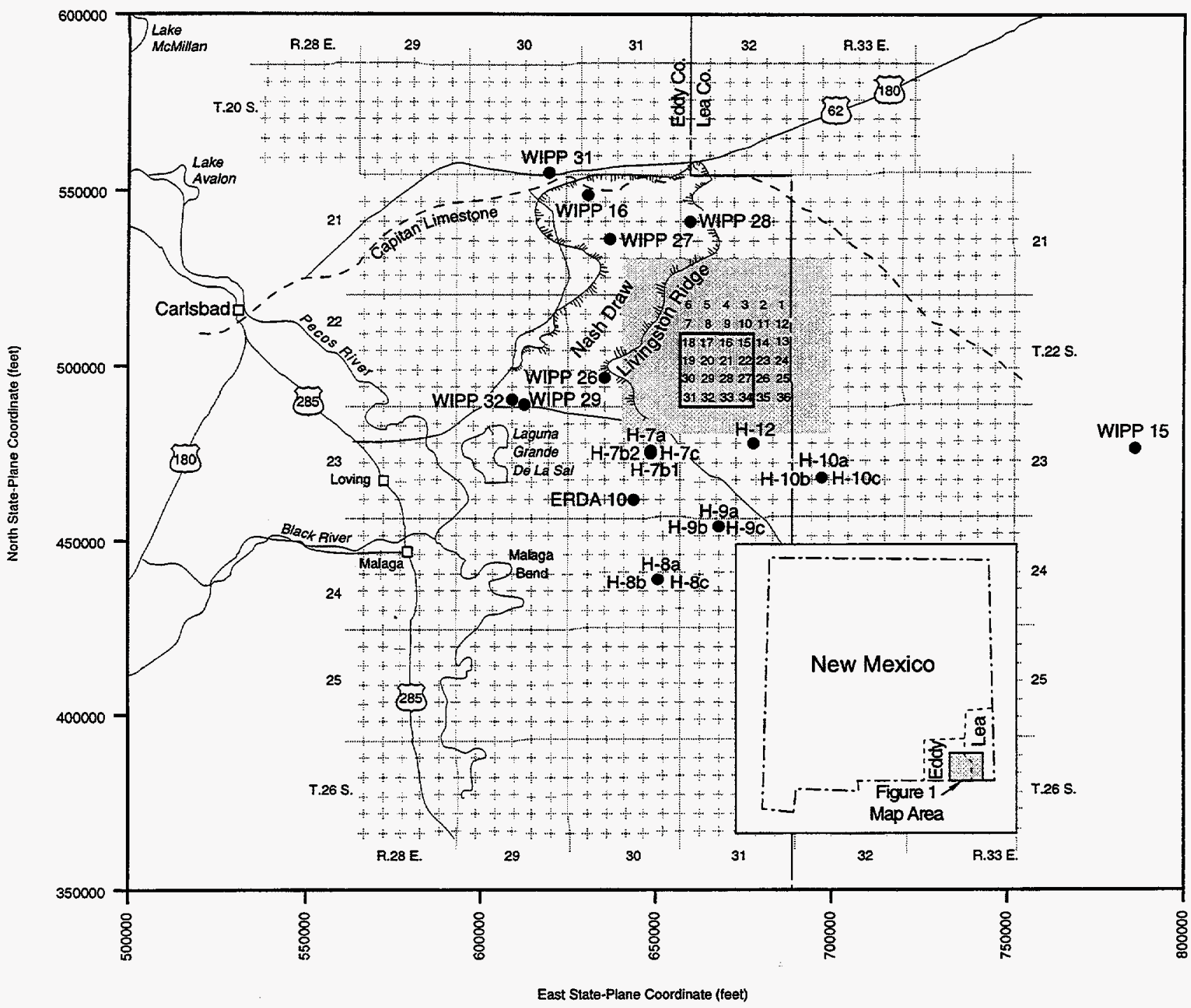

- Section Comer

- Borehole





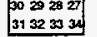

Shaded area enlarged in Figure 2

\section{Shaded area enlarged in Figure 2}

TRI-6747-2-0

Figure 1. Area of the Waste Isolation Pilot Plant regional scale. 


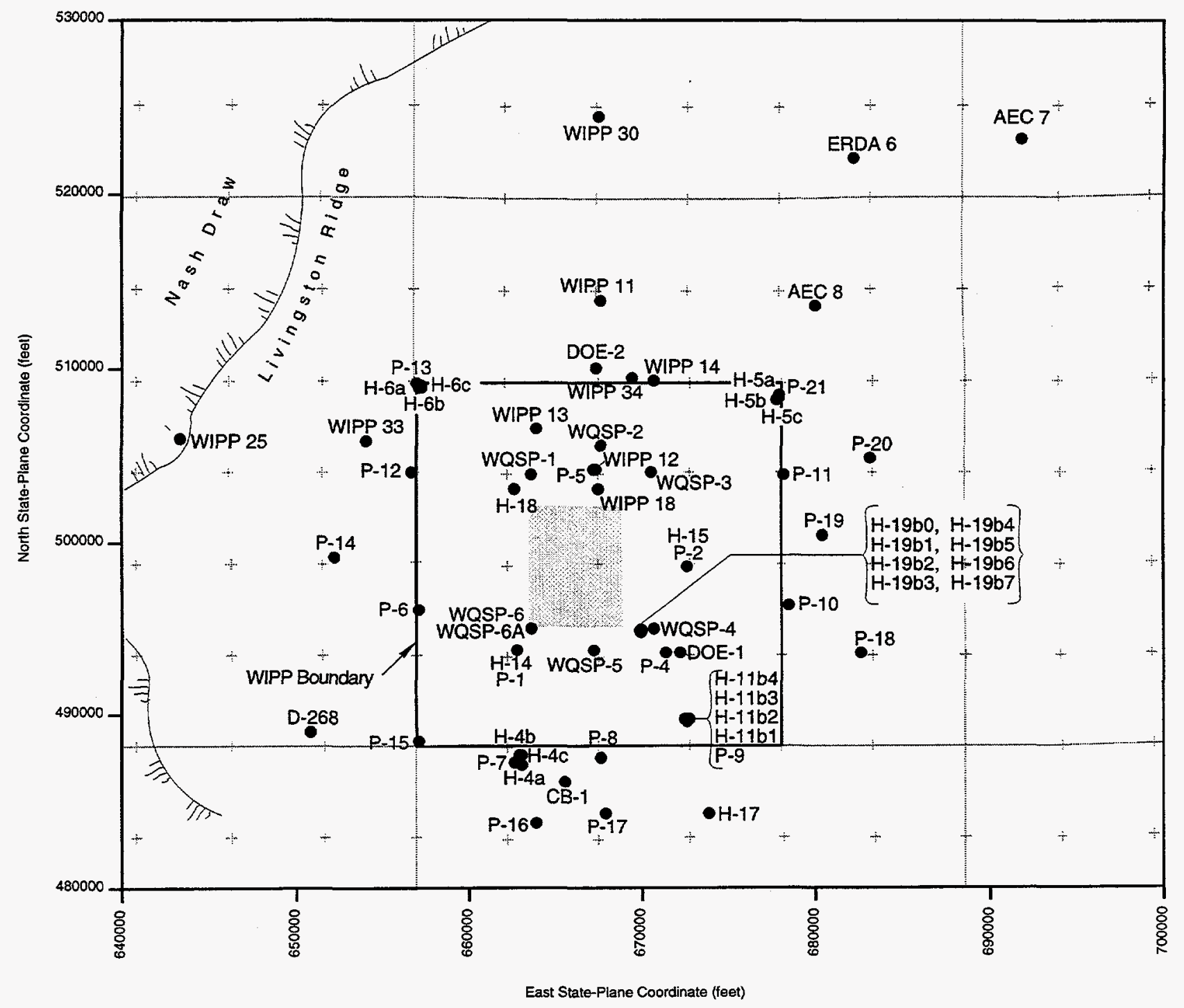

+ Section Comer

- Borehole

Shaded area enlarged in Figure 3

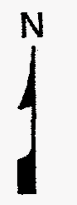

Figure 2. Area of the Waste Isolation Pilot Plant-immediate boundary scale. 


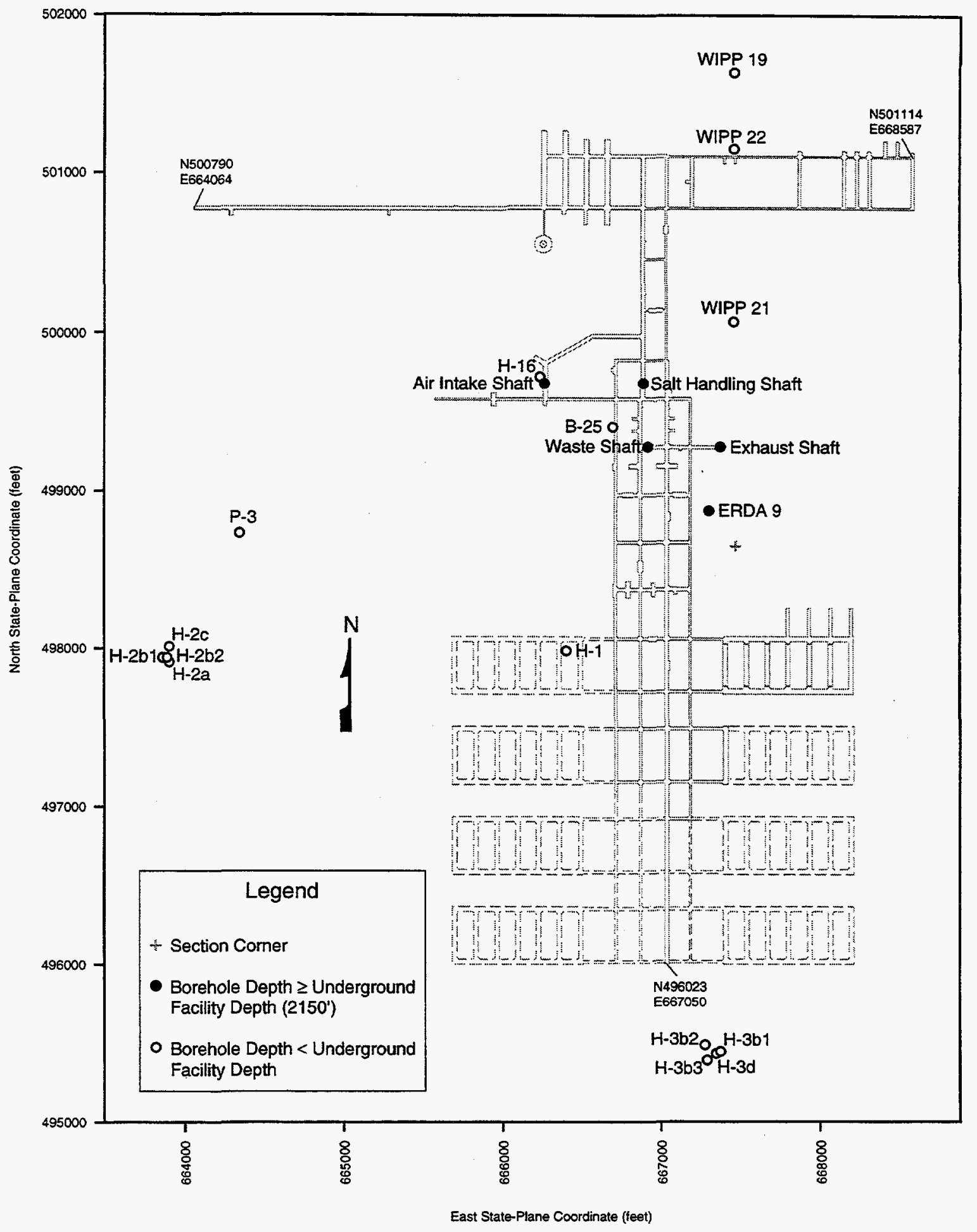

TRI $6747-4-0$

Figure 3. Area of the Waste Isolation Pilot Plant—underground repository scale. 


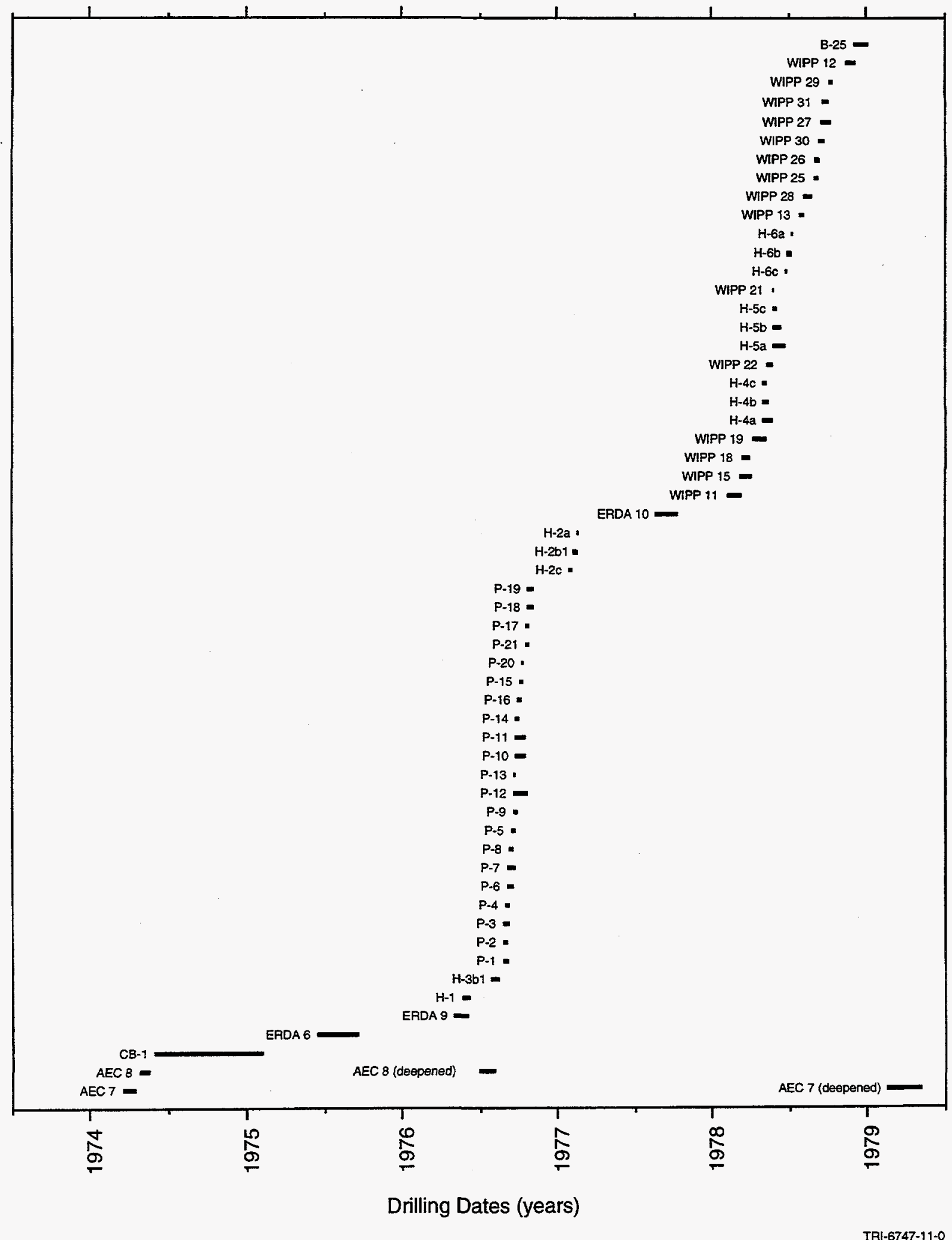

Figure 4. Borehole drilling-time chart, 1974 to 1979. 


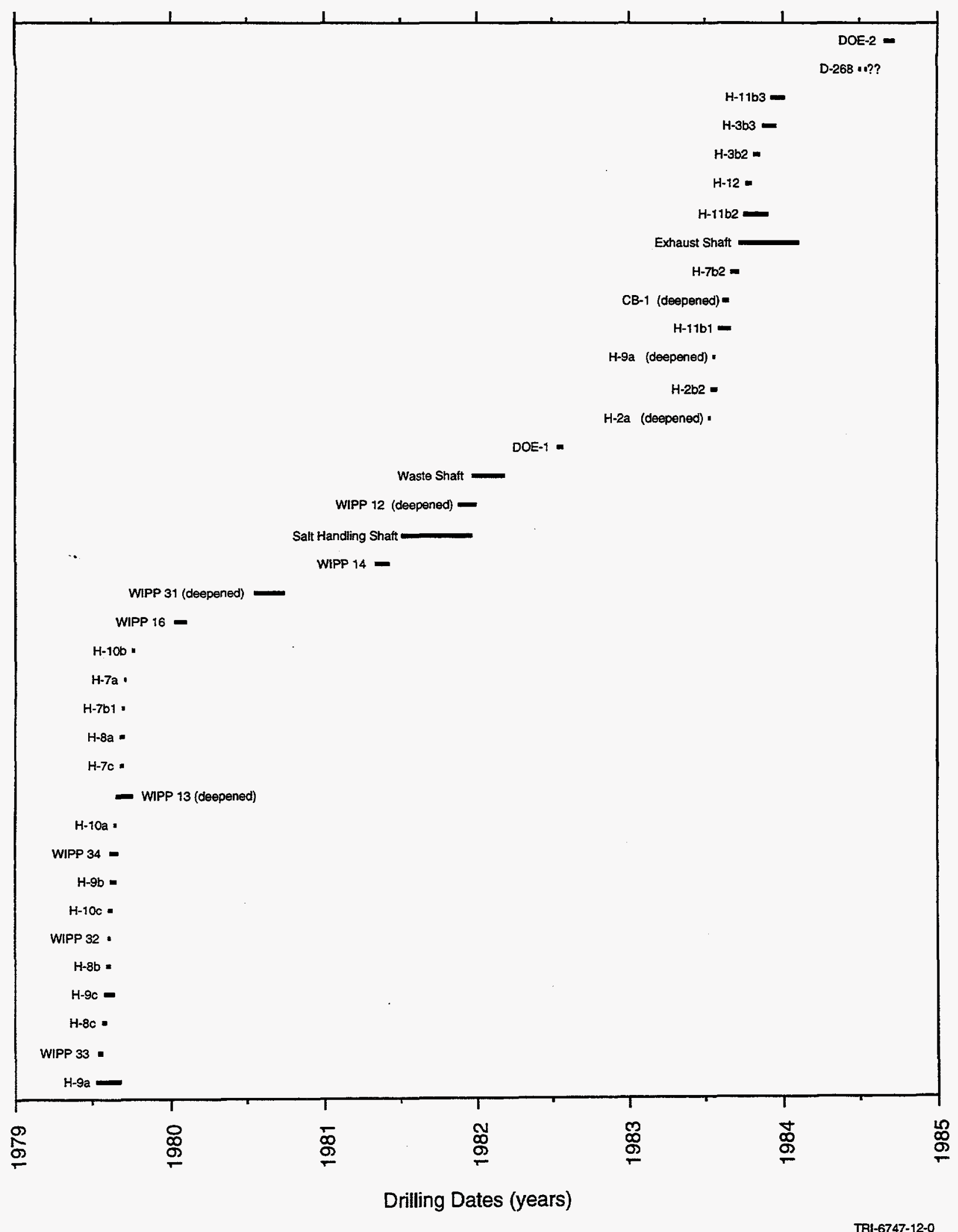

TRI-6747-12-0

Figure 5. Borehole drilling —ime chart, 1979 to 1985. 


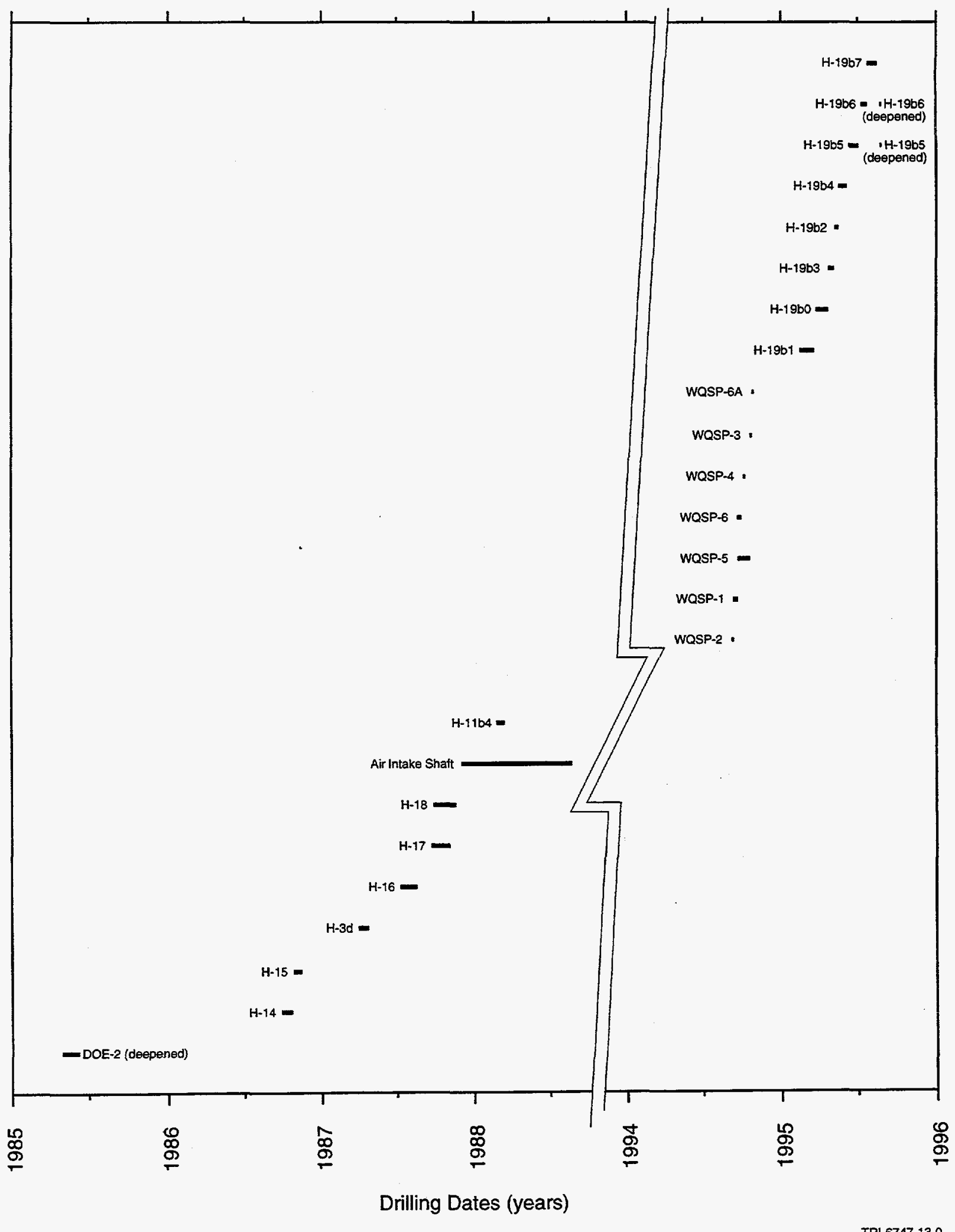

TRI-6747-13-0

Figure 6. Borehole drilling - time chart, 1985 to 1996. 
Page intentionally left blank. 


\section{REFERENCES}

Aguilar, R., L.R. Hill, and G. Newman. 1995. "Integrated, Visually-Effective Graphic Platform of the Waste Isolation Pilot Plant (WIPP) Surface Borehole Data Evaluation," First Biennial TriLaboratory Engineering Conference on Computational Modeling, Pleasanton, CA, October 31-November 2, 1995. SAND95-1310A. Albuquerque, NM: Sandia National Laboratories.

Bachman, G.O. 1980. Regional Geology and Cenozoic History of Pecos Region, Southeastern New Mexico. Open-File Report 80-1099. Denver, CO: U.S. Geological Survey.

Beauheim, R.L. 1989. Interpretation of H-11b4 Hydraulic Tests and the H-11 Multipad Pumping Test of the Culebra Dolomite at the Waste Isolation Pilot Plant (WIPP) Site. SAND89-0536. Albuquerque, NM: Sandia National Laboratories. (Available from the National Technical Information Service (NTIS), Springfield, VA as DE90003307/XAB).

Beauheim, R.L., B.W. Hassinger, and J.A. Klaiber. 1983. Basic Data Report for Borehole Cabin Baby-1 Deepening and Hydrologic Testing, Waste Isolation Pilot Plant (WIPP) Project, Southeastern New Mexico. WTSD-TME-020. Albuquerque, NM: D'Appolonia Consulting Engineers for U.S. Department of Energy. (Available from the National Technical Information Service (NTIS), Springfield, VA as DE86004332/XAB).

Bechtel National, Inc. 1979. "Geologic Data for Borehole B-25.” Document No. 22-V-510-02. San Francisco, CA: Bechtel National, Inc. (Copy on file in the Sandia WIPP Central Files, Sandia National Laboratories, Albuquerque, NM as WPO\#00078.)

Black, S.R. 1982. Basic Data Report Borehole WIPP-12 Deepening, Waste Isolation Pilot Plant (WIPP) Project, Southeastern New Mexico. TME 3148. Albuquerque, NM: D'Appolonia Consulting Engineers, Inc. for U.S. Department of Energy. (Available from the National Technical Information Service (NTIS), Springfield, VA as DE86004343/XAB.)

Christensen, C.L. 1979. Test Plan: Bell Canyon Test WIPP Experimental Program Borehole Plugging. SAND79-0739. Albuquerque, NM: Sandia National Laboratories. (Available from the National Technical Information Service (NTIS), Springfield, VA as SAND-79-0739.)

Christensen, C.L., and E.W. Peterson. 1981. The Bell Canyon Test Summary Report. SAND80-1375. Albuquerque, NM: Sandia National Laboratories. (Available from the National Technical Information Service (NTIS), Springfield, VA as SAND-80-1375.)

D'Appolonia Consulting Engineers, Inc. 1983. Data File Report, ERDA-6 \& WIPP-12 Testing, Addendum 2, Volume VI-ERDA-6 and WIPP-12 Activity Data Files, Activities ERDA-6.11 through 6.14 and WIPP-12.21 through 12.23, Text and Activity Data Files. Albuquerque, NM: Westinghouse Electric Corporation. (Copy on file in the Sandia WIPP Central Files, Sandia National Laboratories, Albuquerque, NM as WPO\#42624.)

Dennehy, K.F. 1982. Results of Hydrologic Tests and Water-Chemistry Analyses, Wells H-6A, H-6B, and H-6C, at the Proposed Waste Isolation Pilot Plant Site, Southeastern New Mexico. WaterResources Investigations 82-8. Albuquerque, NM: U.S. Department of Interior, U.S. Geological Survey. (Available from the National Technical Information Service (NTIS), Springfield, VA as PB83-104042.)

Dennehy, K.F., and J.W. Mercer. 1982. Results of Hydrologic Tests and Water-Chemistry Analyses, Wells $H-5 A, H-5 B$, and H-5C, at the Proposed Waste Isolation Pilot Plant Site, Southeastern New Mexico. Water-Resources Investigations 82-19. Albuquerque, NM: U.S. Department of Interior, U.S. Geological Survey. (Available from the National Technical Information Service (NTIS), Springfield, VA as PB82-263641.) 
Drellack, S.L., Jr., and J.G. Wells. 1982a. Geologic and Well-Construction Data for the H-7 Borehole Complex Near the Proposed Waste Isolation Pilot Plant Site, Southeastern New Mexico. Water-Resources Investigations 82-38. Albuquerque, NM: U.S. Department of Interior, U.S. Geological Survey.

Drellack, S.L., Jr., and J.G. Wells. 1982b. Geologic and Well-Construction Data for the H-9 Borehole Complex Near the Proposed Waste Isolation Pilot Plant Site, Southeastern New Mexico. Water-Resources Investigations 82-4111. Albuquerque, NM: U.S. Department of Interior, U.S. Geological Survey. (Available from the National Technical Information Service (NTIS), Springfield, VA as DE85010117/XAB.)

Freeland, M.H. 1982. Basic Data Report for Borehole DOE-1, Waste Isolation Pilot Plant (WIPP) Project, Southeastern New Mexico. TME 3159. Albuquerque, NM: U.S. Department of Energy. (Available from the National Technical Information Service (NTIS), Springfield, VA as DE86004339/XAB.)

Gonzales, M.M. 1989. Compilation and Comparison of Test-Hole Location Surveys in the Vicinity of the Waste Isolation Pilot Plant Site. SAND88-1065. Albuquerque, NM: Sandia National Laboratories. (Available from the National Technical Information Service (NTIS), Springfield, VA as DE89010522/XAB.)

Griswold, G.B. 1977a. Site Selection and Evaluation Studies of the Waste Isolation Pilot Plant (WIPP), Los Medaños, Eddy County, NM. SAND77-0946. Albuquerque, NM: Sandia National Laboratories. (Available from the National Technical Information Service (NTIS), Springfield, VA as SAND-77-0946. Copy on file in the Sandia WIPP Central Files, Sandia National Laboratories, Albuquerque, NM as WPO $\# 28125$.)

Griswold, G.B., ed. 1977b. Chemical Analysis of Potash-Bearing Horizons from 21 Exploratory Holes Drilled at the Tentative Site for the Waste Isolation Pilot Plant, Eddy County, NM. SAND77-1217. Albuquerque, NM: Sandia National Laboratories. (Available from the National Technical Information Service (NTIS), Springfield, VA as SAND-77-1217. Copy on file in the Sandia WIPP Central Files, Sandia National Laboratories, Albuquerque, NM as WPO\#26768.)

Griswold, G.B., and W.C. McWhirter. 1981. Interpretation of Wireline Geophysical Logs ERDA No. 9 Stratigraphic Test Borehole DOE WIPP Site, Eddy County, New Mexico. SAND80-7119. Albuquerque, NM: Sandia National Laboratories. (Available from the National Technical Information Service (NTIS), Springfield, VA as SAND-80-7119.)

Holt, R.M., and D.W. Powers. 1984. Geotechnical Activities in the Waste Handling Shaft, Waste Isolation Pilot Plant (WIPP) Project, Southeastern New Mexico. WTSD-TME-038. Carlsbad, NM: U.S. Department of Energy, Waste Isolation Pilot Plant. (Available from the National Technical Information Service (NTIS), Springfield, VA as DE86004328/XAB.)

Holt, R.M., and D.W. Powers. 1986. Geotechnical Activities in the Exhaust Shaft. DOE/WIPP 86008. Carlsbad, NM: U.S. Department of Energy. (Copy on file in the Sandia WIPP Central Files, Sandia National Laboratories, Albuquerque, NM as WPO\#41696.)

Holt, R.M., and D.W. Powers. 1988. Facies Variability and Post-Depositional Alteration Within the Rustler Formation in the Vicinity of the Waste Isolation Pilot Plant, Southeastern New Mexico. DOE/WIPP 88-004. Carlsbad, NM: Westinghouse Electric Corporation. 
Holt, R.M., and D.W. Powers. 1990. Geologic Mapping of the Air Intake Shaft at the Waste Isolation Pilot Plant. DOE/WIPP 90-051. Carlsbad, NM: Westinghouse Electric Corporation. (Available from the National Technical Information Service (NTIS), Springfield, VA as DE91017780/XAB.)

Hydro Geo Chem, Inc. 1985. WIPP Hydrology Program Waste Isolation Pilot Plant, SENM Hydrologic Data Report \#1. SAND85-7206. Albuquerque, NM: Sandia National Laboratories. (Available from the National Technical Information Service (NTIS), Springfield, VA as DE86001690/XAB.)

Intera Technologies, Inc. 1986. WIPP Hydrology Program, Waste Isolation Pilot Plant, Southeastern New Mexico Hydrologic Data Report \#3. SAND86-7109. Albuquerque, NM: Sandia National Laboratories. (Available from the National Technical Information Service (NTIS), Springfield, VA as DE86015377/XAB.)

Intera Technologies, Inc. and Hydro Geo Chem, Inc. 1985. WIPP Hydrology Program, Waste Isolation Pilot Plant, Southeastern New Mexico, Hydrologic Data Report \#2. SAND85-7263. Albuquerque, NM: Sandia National Laboratories. (Available from the National Technical Information Service (NTIS), Springfield, VA as DE86007408/XAB.)

Jarolimek, L., M.J. Timmer, and R.F. McKinney. 1983. Geotechnical Activities in the Exploratory Shaft-Selection of the Facility Interval Waste Isolation Pilot Plant (WIPP) Project Southeastern New Mexico. TME 3178. Albuquerque, NM: U.S. Department of Energy, -Waste Isolation Pilot Plant. (Available from the National Technical Information Service (NTIS), Springfield, VA as DE86004323/XAB.)

Jones, C.L. 1978. Test Drilling for Potash Resources: Waste Isolation Pilot Plant Site, Eddy County, New Mexico. Open-File Report 78-592. Denver, CO: U.S. Geological Survey for the U.S. Department of Energy, Albuquerque Operations Office. Vol. 1.

Jones, C.L. 1981a. Geologic Data for Borehole ERDA-6, Eddy County, New Mexico. Open- File Report 81-468. Denver, CO: U.S. Geological Survey. (Available from the National Technical Information Service (NTIS), Springfield, VA as USGS-OFR-81-468.)

Jones, C.L. 1981b. Geologic Data for Borehole ERDA-9, Eddy County, New Mexico. Open- File Report 81-469. Denver, CO: U.S. Geological Survey. (Available from the National Technical Information Service (NTIS), Springfield, VA as USGS-OFR-81-469.)

Mercer, J.W. 1987. Compilation of Hydrologic Data from Drilling the Salado and Castile Formations Near the Waste Isolation Pilot Plant (WIPP) Site in Southeastern New Mexico. SAND86-0954. Albuquerque, NM: Sandia National Laboratories. (Available from the National Technical Information Service (NTIS), Springfield, VA as DE87004553/XAB.)

Mercer, J.W., and B.R. Orr. 1979. Interim Data Report on the Geohydrology of the Proposed Waste Isolation Pilot Plant Site, Southeast New Mexico. Water-Resources Investigations 79-98. Albuquerque, NM: U.S. Geological Survey. (Available from the National Technical Information Service (NTIS), Springfield, VA as PB80-128762.)

Mercer, J.W., and R.P. Snyder. 1990a. Basic Data Report for Drillholes at the H-11 Complex (Waste Isolation Pilot Plant-WIPP). SAND89-0200. Albuquerque, NM: Sandia National Laboratories. (Available from the National Technical Information Service (NTIS), Springfield, VA as DE91002548/XAB.) 
Mercer, J.W., and R.P. Snyder. 1990b. Basic Data Report for Drillhole H-12 (Waste Isolation Pilot Plant-WIPP). SAND89-0201. Albuquerque, NM: Sandia National Laboratories. (Available from the National Technical Information Service (NTIS), Springfield, VA as DE90010322/XAB.)

Mercer, J.W., and R.P. Snyder. 1990c. Basic Data Report for Drillholes H-14 and H-15 (Waste Isolation Pilot Plant - WIPP). SAND89-0202. Albuquerque, NM: Sandia National Laboratories. (Available from the National Technical Information Service (NTIS), Springfield, VA as DE90008233/XAB.)

Mercer, J.W., and R.P. Snyder. 1990d. Basic Data Report for Drillhole H-16 (Waste Isolation Pilot Plant - WIPP). SAND89-0203. Albuquerque, NM: Sandia National Laboratories. (Available from the National Technical Information Service (NTIS), Springfield, VA as DE90008236/XAB.)

Mercer, J.W., and R.P. Snyder. 1990e. Basic Data Report for Drillholes H-17 and H-18 (Waste Isolation Pilot Plant - WIPP). SAND89-0204. Albuquerque, NM: Sandia National Laboratories. (Available from the National Technical Information Service (NTIS), Springfield, VA as DE90008234/XAB.)

Mercer, J.W., P. Davis, K.F. Dennehy, and C.L. Goetz. 1981. Results of Hydrologic Tests and WaterChemistry Analyses, Wells $H-4 A, H-4 B$, and $H-4 C$ at the Proposed Waste Isolation Pilot Plant Site, Southeastern New Mexico. Water-Resources Investigations 81-36. Albuquerque, NM: U.S. Geological Survey. (Available from the National Technical Information Service (NTIS), Springfield, VA as PB82-153610.)

Mercer, J.W., R.L. Beauheim, R.P. Snyder, and G.M. Fairer. 1987. Basic Data Report for Drilling and Hydrologic Testing of Drillhole DOE-2 at the Waste Isolation Pilot Plant (WIPP) Site. SAND86-0611. Albuquerque, NM: Sandia National Laboratories. (Available from the National Technical Information Service (NTIS), Springfield, VA as DE87009350/XAB.)

Munson, D.E., D.L. Hoag, J.R. Ball, G.T. Baird, and R.L. Jones. 1995. Air Intake Shaft Performance Tests (Shaft V): In Situ Data Report (Moy 1988 - July 1995). SAND94-1311. Albuquerque, NM: Sandia National Laboratories. (Available from the National Technical Information Service (NTIS), Springfield, VA as DE96006196/XAB.)

National Academy of Sciences (NAS). 1957. The Disposal of Radioactive Waste on Land: Report of the Committee on Waste Disposal of the Division of Earth Sciences. Publication 519. Washington, DC: National Academy of Sciences - National Research Council. (Available from the National Technical Information Service (NTIS), Springfield, VA as DE92017903/XAB.)

Pearson, F.J., Jr., V.A. Kelley, and J.F. Pickens. 1987. Preliminary Design for a Sorbing Tracer Test in the Culebra Dolomite at the H-3 Hydropad at the Waste Isolation Pilot Plant (WIPP) Site. SAND86-7177. Albuquerque, NM: Sandia National Laboratories. (Available from the National Technical Information Service (NTIS), Springfield, VA as DE87006768/XAB.)

Popielak, R.S., R.L. Beauheim, S.R. Black, W.E. Coons, C.T. Ellingson, and R.L. Olsen. 1983. Brine Reservoirs in the Castile Formation, Waste Isolation Pilot Plant (WIPP) Project,

Southeastern New Mexico. WTSD-TME-3153. Albuquerque, NM: US Department of Energy. (Available from the National Technical Information Service (NTIS), Springfield, VA as DE86004341/XAB.) 
Powers, D.W. 1980. "WIPP Statement of Work for Deepening and Testing WIPP 31." Albuquerque, NM: Sandia National Laboratories. (Copy on file in the Sandia WIPP Central Files, Sandia National Laboratories, Albuquerque, NM as WPO\#07885.)

Powers, D.W. 1996. Tracing Early Breccia Pipe Studies, Waste Isolation Pilot Plant, Southeastern New Mexico: A Study of the Documentation Available and Decision-Making During the Early Years of WIPP. SAND94-0991. Albuquerque, NM: Sandia National Laboratories. (Available from the National Technical Information Service (NTIS), Springfield, VA as DE96004946/XAB .)

Powers, D.W., and R. Holt. 1990. "Sedimentology of the Rustler Formation Near the Waste Isolation Pilot Plant (WIPP) Site," Geological and Hydrological Studies of Evaporites in the Northern Delaware Basin for the Waste Isolation Pilot Plant (WIPP), New Mexico, GSA 1990 Annual Meeting, Field Trip \#14, November 1-4, 1990. Leaders: D.Powers, R. Holt, R.L. Beauheim, and N. Rempe. Dallas, TX: Dallas Geological Society. 79-84. (Copy on file in the Sandia WIPP Central Files, Sandia National Laboratories, Albuquerque, NM as WPO\#41633.)

Powers, D.W., S.J. Lambert, S-E. Shaffer, L.R. Hill, and W.D. Weart, eds. 1978. Geological Characterization Report, Waste Isolation Pilot Plant (WIPP) Site, Southeastern New Mexico. SAND78-1596. Albuquerque, NM: Sandia National Laboratories. Vol. 1. (Available from the National Technical Information Service (NTIS), Springfield, VA as SAND-78-1596.)

Public Law 96-164. 1979. Department of Energy National Security and Military Applications of Nuclear Energy Authorization Act of 1980. (Copy on file in the Sandia WIPP Central Files, Sandia National Laboratories, Albuquerque, NM as WPO\#39103.)

Public Law 102-579. 1992. Waste Isolation Pilot Plant Land Withdrawal Act. (Copy on file in the Sandia WIPP Central Files, Sandia National Laboratories, Albuquerque, NM as WPO\#39105.)

Sanchez, P.E., and P.W. McCasland. 1994. Assessment of Solid Waste Management Units at the Waste Isolation Pilot Plant: Supporting Documentation for RCRA Facility Assessment. NMED/WIPP 93-001, NMED/DOE/AIP-94/1. Carlsbad, NM: New Mexico Environment Department, DOE Environmental Oversight and Environmental Surveillance Section. (Available from Zimmerman Government Publications Department, University of New Mexico, Albuquerque, NM as \#E 1.28:NMED/DOE/AIP-94/1.)

Sandia National Laboratories and D'Appolonia Consulting Engineers. 1982a. Basic Data Report for Drillhole WIPP 12 (Waste Isolation Pilot Plant - WIPP). SAND82-2336. Albuquerque, NM: Sandia National Laboratories. (Available from the National Technical Information Service (NTIS), Springfield, VA as DE83003833.)

Sandia National Laboratories and D'Appolonia Consulting Engineers. 1982b. Basic Data Report for Deepening of Drillhole WIPP 13 (Waste Isolation Pilot Plant - WIPP). SAND82-1880. Albuquerque, NM: Sandia National Laboratories. (Available from the National Technical Information Service (NTIS), Springfield, VA as DE83003782.)

Sandia National Laboratories and D'Appolonia Consulting Engineers. 1982c. Basic Data Report for Drillhole WIPP 14 (Waste Isolation Pilot Plant - WIPP). SAND82-1783. Albuquerque, NM: Sandia National Laboratories. (Available from the National Technical Information Service (NTIS), Springfield, VA as DE82022241.)

Sandia National Laboratories and D'Appolonia Consulting Engineers. 1983a. Basic Data Report for Drillhole AEC 7 (Waste Isolation Pilot Plant - WIPP). SAND79-0268. Albuquerque, NM: Sandia National Laboratories. (Available from the National Technical Information Service (NTIS), Springfield, VA as DE83005889.) 
Sandia National Laboratories and D'Appolonia Consulting Engineers. 1983b. Basic Data Report for Drillhole AEC 8 (Waste Isolation Pilot Plant - WIPP). SAND79-0269. Albuquerque, NM: Sandia National Laboratories. (Available from the National Technical Information Service (NTIS), Springfield, VA as DE83008481.)

Sandia National Laboratories and D'Appolonia Consulting Engineers. 1983c. Basic Data Report for Drillhole ERDA 10 (Waste Isolation Pilot Plant - WIPP). SAND79-0271. Albuquerque, NM: Sandia National Laboratories. (Available from the National Technical Information Service (NTIS), Springfield, VA as DE83010296.)

Sandia National Laboratories and U.S. Geological Survey. 1979a. Basic Data Report for Drillhole WIPP 13 (Waste Isolation Pilot Plant - WIPP). SAND79-0273. Albuquerque, NM: Sandia National Laboratories. (Available from the National Technical Information Service (NTIS), Springfield, VA as SAND-79-0273.)

Sandia National Laboratories and U.S. Geological Survey. 1979b. Basic Data Report for Drillhole WIPP 25 (Waste Isolation Pilot Plant - WIPP). SAND79-0279. Albuquerque, NM: Sandia National Laboratories. (Available from the National Technical Information Service (NTIS), Springfield, VA as SAND-79-0279.)

Sandia National Laboratories and U.S. Geological Survey. 1979c. Basic Data Report for Drillhole WIPP 26 (Waste Isolation Pilot Plant - WIPP). SAND79-0280. Albuquerque, NM: Sandia National Laboratories. (Available from the National Technical Information Service (NTIS), Springfield, VA as SAND-79-0280.)

Sandia National Laboratories and U.S. Geological Survey. 1979d. Basic Data Report for Drillhole WIPP 27 (Waste Isolation Pilot Plant - WIPP). SAND79-0281. Albuquerque, NM: Sandia National Laboratories. (Available from the National Technical Information Service (NTIS), Springfield, VA as SAND-79-0281.)

Sandia National Laboratories and U.S. Geological Survey. 1979e. Basic Data Report for Drillhole WIPP 28 (Waste Isolation Pilot Plant - WIPP). SAND79-0282. Albuquerque, NM: Sandia National Laboratories. (Available from the National Technical Information Service (NTIS), Springfield, VA as SAND-79-0282.)

Sandia National Laboratories and U.S. Geological Survey. 1979f. Basic Data Report for Drillhole WIPP 29 (Waste Isolation Pilot Plant - WIPP). SAND79-0283. Albuquerque, NM: Sandia National Laboratories. (Available from the National Technical Information Service (NTIS), Springfield, VA as SAND-79-0283.)

Sandia National Laboratories and U.S. Geological Survey. 1980a. Basic Data Report for Drillhole WIPP 18 (Waste Isolation Pilot Plant - WIPP). SAND79-0275. Albuquerque, NM: Sandia National Laboratories. (Available from the National Technical Information Service (NTIS), Springfield, VA as SAND-79-0275.)

Sandia National Laboratories and U.S. Geological Survey. 1980b. Basic Data Report for Drillhole WIPP 19 (Waste Isolation Pilot Plant - WIPP). SAND79-0276. Albuquerque, NM: Sandia National Laboratories. (Available from the National Technical Information Service (NTIS), Springfield, VA as SAND-79-0276.)

Sandia National Laboratories and U.S. Geological Survey. 1980c. Basic Data Report for Drillhole WIPP 21 (Waste Isolation Pilot Plant - WIPP). SAND79-0277. Albuquerque, NM: Sandia National Laboratories. (Available from the National Technical Information Service (NTIS), Springfield, VA as SAND-79-0277.) 
Sandia National Laboratories and U.S. Geological Survey. 1980d. Basic Data Report for Drillhole WIPP 22 (Waste Isolation Pilot Plant - WIPP). SAND79-0278. Albuquerque, NM: Sandia National Laboratories. (Available from the National Technical Information Service (NTIS), Springfield, VA as SAND-79-0278.)

Sandia National Laboratories and U.S. Geological Survey. 1980e. Basic Data Report for Drillhole WIPP 30 (Waste Isolation Pilot Plant - WIPP). SAND79-0284. Albuquerque, NM: Sandia National Laboratories. (Available from the National Technical Information Service (NTIS), Springfield, VA as SAND-79-0284.)

Sandia National Laboratories and U.S. Geological Survey. 1980f. Basic Data Report for Drillhole WIPP 32 (Waste Isolation Pilot Plant - WIPP). SAND80-1102. Albuquerque, NM: Sandia National Laboratories. (Available from the National Technical Information Service (NTIS), Springfield, VA as SAND-80-1102.)

Sandia National Laboratories and U.S. Geological Survey. 1981a. Basic Data Report for Drillhole WIPP 33 (Waste Isolation Pilot Plant - WIPP). SAND80-2011. Albuquerque, NM: Sandia National Laboratories. (Available from the National Technical Information Service (NTIS), Springfield, VA as SAND-80-2011.)

Sandia National Laboratories and U.S. Geological Survey. 1981b. Basic Data Report for Drillhole WIPP 34 (Waste Isolation Pilot Plant - WIPP). SAND81-2643. Albuquerque, NM: Sandia National Laboratories. (Available from the National Technical Information Service (NTIS), Springfield, VA as DE82008343.)

Sandia National Laboratories and U.S. Geological Survey. 1982. Basic Data Report for Drillhole WIPP 11 (Waste Isolation Pilot Plant - WIPP). SAND79-0272. Albuquerque, NM: Sandia National Laboratories. (Available from the National Technical Information Service (NTIS), Springfield, VA as DE82012053/XAB.)

Sandia National Laboratories and U.S. Geological Survey. 1983a. Basic Data Report for Drillhole ERDA 6 (Waste Isolation Pilot Plant - WIPP). SAND79-0267. Albuquerque, NM: Sandia National Laboratories. (Available from the National Technical Information Service (NTIS), Springfield, VA as DE83006392.)

Sandia National Laboratories and U.S. Geological Survey. 1983b. Basic Data Report for Drillhole ERDA 9 (Waste Isolation Pilot Plant - WIPP). SAND79-0270. Albuquerque, NM: Sandia National Laboratories. (Available from the National Technical Information Service (NTIS), Springfield, VA as DE83006393.)

Sandia National Laboratories and University of New Mexico. 1981. Basic Data Report for Drillhole WIPP 15 (Waste Isolation Pilot Plant - WIPP). SAND79-0274. Albuquerque, NM: Sandia National Laboratories. (Available from the National Technical Information Service (NTIS), Springfield, VA as DE82006868.)

Saulnier, G.J., Jr., G.A. Freeze, and W.A. Stensrud. 1986. WIPP Hydrology Program, Waste Isolation Pilot Plant, Southeastern New Mexico. Hydrologic Data Report \#4. SAND86-7166. Albuquerque, NM: Sandia National Laboratories. (Available from the National Technical Information Service (NTIS), Springfield, VA as DE87005975/XAB.)

Seward, P.D., comp. 1982. Abridged Borehole Histories for the Waste Isolation Pilot Plant (WIPP) Studies. SAND82-0080. Albuquerque, NM: Sandia National Laboratories. (Available from the National Technical Information Service (NTIS), Springfield, VA as DE82009348.) 
Snyder, R.P., and L.M. Gard, Jr. 1982. Evaluation of Breccia Pipes in Southeastern New Mexico and Their Relation to the Waste Isolation Pilot Plant (WIPP) Site, With Section on Drill-Stem Tests, WIPP 31, by J.W. Mercer. Open-File Report 82-968. Denver, CO: U.S. Geological Survey for the Albuquerque Operations Office, U.S. Department of Energy.

Statler, R.D. 1978. "Field Operations Program of Sandia Labs WIPP Site Investigations Resistivity Anomaly. Exploratory Well: WIPP 31." Albuquerque, NM: Sandia National Laboratories. (Copy on file in the Sandia WIPP Central Files, Sandia National Laboratories, Albuquerque, NM as WPO\#02732.)

Stensrud, W.A., M.A. Bame, K.D. Lantz, A.M. LaVenue, J.B. Palmer, and G.J. Saulnier, Jr. 1987. WIPP Hydrology Program, Waste Isolation Pilot Plant, Southeastern New Mexico. Hydrologic Data Report \#5. Parts: A. WIPP-13 Multipad Test. B. H-4c, P-17, ERDA-9, and Cabin Baby-1 Slug Tests. C. Engle and Carper Well Pumping Tests. D. WIPP-12, H14, and H-15 Drill-Stem Tests. E. Water-Level Data. SAND87-7125. Albuquerque, NM: Sandia National Laboratories. (Available from the National Technical Information Service (NTIS), Springfield, VA as DE88003613/XAB.)

Stensrud, W.A., M.A. Bame, K.D. Lantz, T.L Cauffman, J.B. Palmer, and G.J. Saulnier, Jr. 1988a. WIPP Hydrology Program, Waste Isolation Pilot Plant, Southeastern New Mexico. Hydrologic Data Report \#6. Parts: A Waste-Handling Shaft Pulse Testing. B. H-1, H-12, and P-15 Slug Tests. C. H-2 Pumping Test. D. H-16, H-17, and H-18 Drill-Stem, Slug, and Pulse Tests. E. Water-Level Data. SAND87-7166. Albuquerque, NM: Sandia National Laboratories. (Available from the National Technical Information Service (NTIS), Springfield, VA as DE88013135/XAB.)

Stensrud, W.A., M.A. Bame, K.D. Lantz, J.B. Palmer, and G.J. Saulnier, Jr. 1988b. WIPP Hydrology Program, Waste Isolation Pilot Plant, Southeastern New Mexico. Hydrologic Data Report \#7. Parts: A. WIPP-30, WIPP-12, and P-18 Slug Tests. B. H-11b4 Drill-Stem, Slug, and Pumping Tests. C. H-18 Pumping Test. D. Water-Level Data. SAND88-7014. Albuquerque, NM: Sandia National Laboratories. (Available from the National Technical Information Service (NTIS), Springfield, VA as DE89004642/XAB.)

Stensrud, W.A., M.A. Bame, K.D. Lantz, J.B. Palmer, and G.J. Saulnier, Jr. 1990. WIPP Hydrology +Program, Waste Isolation Pilot Plant, Southeastern New Mexico. Hydrologic Data Report \#8. Parts: A. H-11 Multipad Pumping and Convergent-Flow Tracer Test. B. P-14 Air-Lift Pumping Test. C. AEC-7 and D-268 Slug Tests. D. H-2b1, H-3b1, and H-3d Slug and Pulse Tests. E. Hydraulic Effects of Air-Intake Shaft Construction. F. Water-Level Data. SAND89-7056. Albuquerque, NM: Sandia National Laboratories. (Available from the National Technical Information Service (NTIS), Springfield, VA as DE90014708/XAB.)

U.S. Department of Energy. 1980. Final Environmental Impact Statement: Waste Isolation Pilot Plant. DOE/EIS-0026. Washington D.C.: United States Department of Energy. Vols. 1-2. (Available from the National Technical Information Service (NTIS), Springfield, VA as DOE/EIS-0026.)

U.S. Department of Energy. 1994. Experimental Program Plan for the Waste Isolation Pilot Plant. DOE/WIPP 94-008, Revision 0. Carlsbad, NM: United States Department of Energy, Carlsbad Area Office. (Available from the National Technical Information Service (NTIS), Springfield, VA as DE94007975/XAB.) 
U.S. Department of Energy. 1995a. Waste Isolation Pilot Plant Borehole Data. DOE/WIPP-952092. Carlsbad, NM: Prepared for United States Department of Energy, Carlsbad Area Office by Westinghouse Electric Corporation, Waste Isolation Division. (Available from the National Technical Information Service (NTIS), Springfield, VA as DE95012971/XAB.)

U.S. Department of Energy. 1995b. Basic Data Report for Wells WQSP 1 through WQSP 6 a. DOE/WIPP-95-2154. Carlsbad, NM: Westinghouse Electric Corporation, Waste Isolation Division. (Available from the National Technical Information Service (NTIS), Springfield, VA as DE96010604/XAB.)

Wells, J.G., and S.L. Drellack, Jr. 1982. Geologic and Well-Construction Data for the H-8 Borehole Complex Near the Proposed Waste Isolation Pilot Plant Site, Southeastern New Mexico. Water-Resources Investigations 82-4118. Albuquerque, NM: U.S. Geological Survey. (Available from the National Technical Information Service (NTIS), Springfield, VA as DE85010116/XAB.)

Wells, J.G., and S.L. Drellack, Jr. 1983. Geologic and Well-Construction Data for the H-10 Borehole Complex Near the Proposed Waste Isolation Pilot Plant Site, Southeastern New Mexico. Water-Resources Investigations 83-4124. Albuquerque, NM: U.S. Geological Survey. (Available from the National Technical Information Service (NTIS), Springfield, VA as DE85009020/XAB.) 
Page intentionally left blank. 


\section{APPENDIX A: MEMORANDA REGARDING REFERENCED DATA}

\section{Referenced Memoranda}

Jerry W. Mercer, January 22, 1991

A-3

Jerry W. Mercer, October 26, 1994

A-14

Jerry W. Mercer, July 18, 1996

A-15 
Page intentionally left blank.

$$
\text { A-2 }
$$


Date: January 22,1991

To: $\quad$ Wendell D. Weart, 6340

From: Jerry W. Mercer, 9333 Gerry W Wuener

Subject: Test Holes Drilled in Support of Geotechnical'Studies at the WIPP Site

This memo is written concerning the ultimate disposition of the "permitted" test holes that have been drilled in support of the geotechnical evaluation of the WIPP site. The concern comes primarily in the area of the "ownership" of these test holes, herein called the legal operator. This is the term used in the documents that cover the permitting of these wells. As you know, permitting of these test holes requires either a drilling permit from the U.S. Geological Survey as was done in the potash drilling program, or a drilling permit from the State Engineers Office (SEO), as was done for those holes drilled for geologic exploration and hydrologic testing. All of these wells have been drilled in the Carlsbad Closed Groundwater Basin, closed to un-permitted drilling some years ago by the New Mexico SEO. In addition to a drilling permit, most of these test holes were drilled on land under the control of the Bureau of Land Management, which also requires a land use permit.

When Sandia acquired these permits to drill these test holes, there were certain legal obligations that went along with the permit. These obligations for the drilling permits require keeping the well in good condition during use and providing assurance that the water producing zones are kept properly isolated. The additional requirement of proper plugging during abandonment is also the obligation of the legal operator. I realize that most of these wells presently in use will be kept for "long-term monitoring," but the legal obligations are still Sandia's. The legal requirements placed on restoration of the well access roads and drilling locations in most cases are also Sandia's. These requirements include restoring the roads and locations back to the pre-drilling conditions. It should be noted that for some of the abandoned wells this has already been done.

I believe we need to evaluate these wells that fall under these requirements and determine the direction we want to take. I would suggest that we discuss this with Rick Beauheim and others that may have direct involvement and get their opinions. May be at such time as Sandia is no longer in need of these wells (or control as the case may be), Sandia could eventually transfer the legal responsibility to the DOE. At least we should let them know that restoration of these wells at some future time will have to be done. This not only is a legal obligation, but from Sandia's standpoint, could be an economic one as plugging and abandoning these well sites could be very expensive.

I think we should also consider providing the SEO and the Bureau of Land Management a letter that would tell them that we intend to use these wells for an extended time as monitoring wells (if that is the case). Some of these wells have 
been in this status for almost 15 years and I don't think any follow up paper work has been sent concerning this issue.

I have included, as an attachment, a table of the wells drilled at the WIPP site and adjacent areas that the current hole status (as I know it to be), permit number, and "ownership" status. Perhaps we can sit down sometime and discuss this matter. At this time I do not see an extreme urgency, but it should be done in the near future.

Additional Distribution:

$6344 \quad$ R. L. Beauheim

9333 O. L. Burchett 
H-3 Hydropad Boreholes at the WIPP Site - Data from J.W. Mercer Field Notes

\begin{tabular}{|c|c|c|c|c|c|c|c|}
\hline $\begin{array}{l}\text { Borehole } \\
\text { I.D }\end{array}$ & $\begin{array}{l}\text { Drill Date - } \\
\text { Start/End }\end{array}$ & $\begin{array}{l}\text { Purpose for } \\
\text { Drilling }\end{array}$ & $\begin{array}{l}\text { Total } \\
\text { Depth } \\
\text { (ft) }\end{array}$ & $\begin{array}{l}\text { \# Cores } \\
\text { Extracted }\end{array}$ & $\begin{array}{l}\text { Cored } \\
\text { Interval(s) }\end{array}$ & References & Comments \\
\hline H-3b2 & $\begin{array}{l}10 / 25 / 83 \\
11 / 04 / 83\end{array}$ & $\begin{array}{l}\text { Hydro- } \\
\text { monitoring }\end{array}$ & 725 & 32 & $32-725$ & $\begin{array}{l}\text { SAND86-7177, } \\
\text { Pearson et al. } 1987\end{array}$ & $\begin{array}{l}\text { Hole completed in the Culebra } \\
\text { Dolomite of the Rustler Fm for } \\
\text { future tracer test. }\end{array}$ \\
\hline $\mathrm{H}-3 \mathrm{~b} 3$ & $\begin{array}{l}11 / 15 / 83 \\
01 / 30 / 84\end{array}$ & $\begin{array}{l}\text { Hydro- } \\
\text { monitoring }\end{array}$ & 730 & 44 & $34-730$ & $\begin{array}{l}\text { SAND86-7177, } \\
\text { Pearson et al. } 1987\end{array}$ & $\begin{array}{l}\text { Hole completed in the Culebra } \\
\text { Dolomite of the Rustler Fm for } \\
\text { future tracer test. }\end{array}$ \\
\hline$H-3 d$ & $\begin{array}{l}03 / 31 / 87 \\
04 / 22 / 87\end{array}$ & $\begin{array}{l}\text { Hydro- } \\
\text { monitoring }\end{array}$ & 554 & N/A; none & N/A; none & $\begin{array}{l}\text { SAND86-7177, } \\
\text { Pearson et al. } 1987\end{array}$ & $\begin{array}{l}\text { Hole is open from bottom of surface } \\
\text { casing at } 39 \text { feet. Hole is completed } \\
\text { to test Dewy Lake Redbeds Fm and } \\
\text { is used as a water level observation } \\
\text { well. This borehole was originally } \\
\text { called H-3 (b4). }\end{array}$ \\
\hline
\end{tabular}


Attachment 1:

TABLE OF WIPP HOLE STATUS AS OF JANUARY 10, 1991:

Legend:

Underlined hole No. as well as double star (**) indicates well that is currently being used as a monitoring well.

Legal operator: (SNL)--Sandia National Laboratories (Westin.)--Westinghouse (DOE) --Departøent of Energy

Star $(*)$ by location and elevation of well indicates the monument location and elevation, not of the well itself.

Permit No. (SEO) stands for State Engineers Office, (USGS) stands for the U. S. Geological Survey. Date of permit included.

Hole Purpose: (GE)--Geologic Esploration (HTH)--Hydrologic Test Hole (PE)--Potash Evaluation

Hole Status: (PA)--Plugged and abandoned with date (HSC)--Hole or part of hole in standby condition

(HMW)--Hydrologic monitoring well

\begin{tabular}{|c|c|c|c|c|c|c|c|}
\hline Hole No. & DATE OF COMPLETION & Elevation & $\frac{\text { Legal }}{\text { Operator }}$ & $\frac{\text { Total }}{\text { Depth }}$ & $\frac{\text { Permit }}{\text { No. (SEO) }}$ & $\frac{\text { Hole }}{\text { Purpose }}$ & $\frac{\text { Hole }}{\text { status }}$ \\
\hline$P-1$ & $\begin{array}{l}\text { T22S R31E Sec } 29 \\
327 \mathrm{FSL}, 551 \text { FWL } \\
(9 / 02 / 76)\end{array}$ & 3344.8 & SNL & 1591 & $\begin{array}{l}\text { USGS } \\
8 / 18 / 76\end{array}$ & PE & $\begin{array}{l}\text { PA (y) } \\
9 / 2 / 76\end{array}$ \\
\hline P-2 & $\begin{array}{l}\text { T22S R31E Sec } 28 \\
125 \text { FNL, } 172 \text { FEL } \\
(9 / 03 / 76)\end{array}$ & 3477.7 & SNL & 1895 & $\begin{array}{l}\text { USGS } \\
8 / 18 / 76\end{array}$ & $P E$ & $\begin{array}{l}\text { PA (y) } \\
9 / 3 / 76\end{array}$ \\
\hline$P-3$ & $\begin{array}{c}\text { T22S R31E Sec } 20 \\
103 \mathrm{FSI}, 3122 \text { FEL } \\
(9 / 08 / 76)\end{array}$ & 3382.3 & SNL & 1676 & $\begin{array}{l}\text { USGS } \\
8 / 18 / 76\end{array}$ & $P E$ & $\begin{array}{l}\text { PA (y) } \\
9 / 8 / 76\end{array}$ \\
\hline$P-4$ & $\begin{array}{l}\text { T22S R31E Sec } 28 \\
\text { 146 FSL, } 1487 \text { FEL } \\
(9 / 07 / 76)\end{array}$ & 3441.2 & SNL & 1857 & $\begin{array}{l}\text { USGS } \\
8 / 18 / 76\end{array}$ & PE & $\begin{array}{l}\text { PA (y) } \\
9 / 7 / 76\end{array}$ \\
\hline$P-5$ & $\begin{array}{l}\text { T22S R31E Sec } 17 \\
202 \mathrm{FSL}, 165 \mathrm{FEL} \\
(9 / 22 / 76)\end{array}$ & 3471.8 & SNL & 1830 & $\begin{array}{l}\text { USGS } \\
8 / 18 / 76\end{array}$ & PE & $\begin{array}{l}\text { PA (y) } \\
9 / 22 / 76\end{array}$ \\
\hline$P-6$ & $\begin{array}{l}\text { T22S R31E Sec } 30 \\
2767 \text { FSL, } 199 \text { FWL } \\
(9 / 17 / 76)\end{array}$ & 3353.5 & SNL & 1573 & $\begin{array}{l}\text { USGS } \\
8 / 18 / 76\end{array}$ & $\mathrm{PE}$ & $\begin{array}{l}P A(y) \\
9 / 17 / 76\end{array}$ \\
\hline$P-7$ & $\begin{array}{l}\text { T22S R31E Sec } 5 \\
\text { S13 FNL, } 396 \text { FWL } \\
(9 / 22(76)\end{array}$ & 3331.7 & SNL & 1574 & $\begin{array}{l}\text { USGS } \\
8 / 18 / 76\end{array}$ & $\mathrm{PE}$ & $\begin{array}{l}\text { PA (y) } \\
9 / 22 / 76\end{array}$ \\
\hline
\end{tabular}




\begin{tabular}{|c|c|c|c|c|c|c|c|}
\hline Hole No. & $\begin{array}{l}\text { LOCATION \& } \\
\text { DATE OF COMPLETION }\end{array}$ & Elevation & $\frac{\text { Legal }}{\text { operator }}$ & $\frac{\text { Total }}{\text { Depth }}$ & $\frac{\text { Permit }}{\text { No. (SEO) }}$ & Puxpose & $\frac{\text { Hole }}{\text { status }}$ \\
\hline$P-8$ & $\begin{array}{l}\text { T22S R31E Sec } 4 \\
642 \text { FNL, } 96 \text { FWL } \\
(9 / 15 / 76)\end{array}$ & 3336.4 & SNL & 1660 & $\begin{array}{c}\text { USGS } \\
8 / 18 / 76\end{array}$ & $P E$ & $\begin{array}{l}P A(y) \\
9 / 15 / 76\end{array}$ \\
\hline$P-9$ & $\begin{array}{l}\text { T22S R31E Sec } 33 \\
1493 \text { FSL, } 143 \text { FEI } \\
(9 / 26 / 76)\end{array}$ & 3408.6 & SNL & 1796 & $\begin{array}{l}\text { USGS } \\
8 / 18 / 76\end{array}$ & PE & $\begin{array}{l}\text { PA }(y) \\
9 / 26 / 76\end{array}$ \\
\hline$P-10$ & $\begin{array}{c}\text { T22S R31E Sec } 26 \\
2315 \text { FNL, } 339 \text { FWL } \\
(10 / 17 / 76)\end{array}$ & 3508.6 & SNL & 2009 & $\begin{array}{l}\text { USES } \\
8 / 18 / 76\end{array}$ & $P E$ & $\begin{array}{c}\text { PA }(y) \\
10 / 17 / 76\end{array}$ \\
\hline$P-11$ & $\begin{array}{l}\text { I22S R31E Sec } 23 \\
175 \text { FNL, } 177 \text { FHI } \\
(10 / 18 / 76)\end{array}$ & 3505.8 & SNL & 1940 & $\begin{array}{l}\text { USGS } \\
8 / 18 / 76\end{array}$ & PE & $\begin{array}{c}\mathrm{PA}(y) \\
10 / 18 / 76\end{array}$ \\
\hline$P-12$ & $\begin{array}{l}\text { T22S R3OE Sec } 24 \\
167 \text { FWL, } 195 \text { FWL } \\
(10 / 23 / 76)\end{array}$ & 3376.0 & SNL & 1598 & $\begin{array}{l}\text { USGS } \\
8 / 18 / 76\end{array}$ & $\mathrm{PE}$ & $\begin{array}{c}\mathrm{PA}(y) \\
10 / 23 / 76\end{array}$ \\
\hline$P-13$ & $\begin{array}{l}\text { T22S R31E Sec } 18 \\
125 \text { FNL, } 116 \text { FWL } \\
(9 / 24 / 76)\end{array}$ & 3345.4 & SNL & 1576 & $\begin{array}{l}\text { USGS } \\
8 / 18 / 76\end{array}$ & $P E$ & $\begin{array}{c}\text { PA (y) } \\
9 / 17 / 76\end{array}$ \\
\hline$P-14 \div 3$ & $\begin{array}{l}\text { T22S R3OE Sec } 24 * \\
306.6 \mathrm{FSI}, 611.8 \mathrm{FWL} \\
(10 / 04 / 76)\end{array}$ & -3359.76. & SNL & 7.545 & $\begin{array}{l}\text { USES } \\
8 / 18 / 76\end{array}$ & PE & $\begin{array}{l}\text { PA to (y) } \\
759 \text { (HNW })\end{array}$ \\
\hline$\underline{p-15 * *}$ & $\begin{array}{l}\text { T22S R31E Sec } 31 * \\
409.47 \text { FSL, } 188.34 \text { FWL } \\
(10 / 15 / 76)\end{array}$ & $3309.84 *$ & SNL & 1465 & $\begin{array}{l}\text { USGS } \\
8 / 18 / 76\end{array}$ & $P E$ & $\begin{array}{l}\text { PA to (y) } \\
620(\text { HKW })\end{array}$ \\
\hline$P-16$ & $\begin{array}{l}\text { T23S R31E Sec } 5 \\
951 \text { FSL, 1629 FWL } \\
(10 / 06 / 76)\end{array}$ & 3322.8 & SNL & 1585 & $\begin{array}{l}\text { USGS } \\
8 / 18 / 76\end{array}$ & $P E$ & $\begin{array}{l}\text { PA }(y) \\
10 / 6 / 76\end{array}$ \\
\hline$\underline{P}-17 x+2$ & $\begin{array}{l}\text { T23S R3IE Sec } 4 * \\
1371.9 \text { FSL, } 397.7 \text { FWL } \\
(10 / 28 / 76)\end{array}$ & $3335.77 *$ & SNL & 1660 & $\begin{array}{l}\text { USGS } \\
8 / 18 / 76\end{array}$ & $P E$ & $\begin{array}{l}\text { PA to }(y) \\
731 \text { (Hi: })\end{array}$ \\
\hline$P-18 \div$ & $\begin{array}{l}\text { I22S R31E Sec } 26 * \\
136.7 \mathrm{FSL}, 736.5 \mathrm{FEL} \\
\quad(11 / 06 / 76)\end{array}$ & $3477.30 \neq$ & SNL & 1998 & $\begin{array}{l}\text { USGS } \\
8 / 18 / 76\end{array}$ & PE & $\begin{array}{l}\text { PA to (y) } \\
1120 \text { (HIT) }\end{array}$ \\
\hline$P-19$ & $\begin{array}{l}\text { T22S R3IE Sec } 23 \\
1652 \text { FSI, } 2330 \text { FEL } \\
\quad(11 / 04 / 76)\end{array}$ & 3546.3 & SNL & 2000 & $\begin{array}{l}\text { USGS } \\
8 / 18 / 76\end{array}$ & PE & $\begin{array}{l}P A(y) \\
11 / 4 / 76\end{array}$ \\
\hline$P-20$ & $\begin{array}{l}\text { T22S R31E Sec } 14 \\
794 \mathrm{FSL}, 103 \mathrm{FEL} \\
(10 / 15 / 76)\end{array}$ & 3552.9 & SNL & 1995 & $\begin{array}{l}\text { USGS } \\
8 / 18 / 76\end{array}$ & PE & $\begin{array}{l}\text { PA }(y) \\
10 / 15 / 76\end{array}$ \\
\hline
\end{tabular}




\begin{tabular}{|c|c|c|c|c|c|c|c|c|}
\hline Hole No. & $\begin{array}{l}\text { LOCATTON \& } \\
\text { DATE OF COMPLETION }\end{array}$ & Elevation & & $\frac{\text { Legal }}{\text { Operator }}$ & Total & $\frac{\text { Permit }}{\text { No.(SEO) }}$ & $\frac{\text { Hole }}{\text { Purpose }}$ & $\frac{\text { Hole }}{\text { Status }}$ \\
\hline$P-21$ & $\begin{array}{l}\text { T22S R31E Sec IS } \\
852 \text { FNL, 1S0 FEL } \\
(10 / 27 / 76)\end{array}$ & 3510.2 & & SNL & 1915 & $\begin{array}{l}\text { USGS } \\
8 / 18 / 76\end{array}$ & PE & $\begin{array}{l}\text { PA (y) } \\
10 / 27 / 76\end{array}$ \\
\hline$\frac{\text { AEC-7 } * x}{2038 .}$ & $\begin{array}{l}\text { T2IS R32E Sec } 31 * \\
97 \text { FNL, 2037.26 FEL } \\
(4 / 19 / 80)\end{array}$ & 3655.54 & $\Rightarrow$ & SNL & 4734 & $\begin{array}{l}\text { USGS } \\
1 / 12 / 79 \& \\
\text { SEO 辈 } \\
0.08 .1323\end{array}$ & GE & $\begin{array}{l}\text { HSC \& } \\
\text { HMW }\end{array}$ \\
\hline$\underline{A E C-8 \neq-2}$ & $\begin{array}{l}\text { T22S R31E Sec } 11 \\
935 \mathrm{FNL}, 1979 \text { FWI } \\
(8 / 05 / 76)\end{array}$ & 3532 & & SNL & 4911 & ? & $\mathrm{GE}$ & $\begin{array}{l}\text { HSC \& } \\
\text { HMW }\end{array}$ \\
\hline ERDA- 6 & $\begin{array}{l}\text { T21S R31E Sec } 35 \\
2152 \text { FSL, } 910 \text { FEI } \\
\quad(12 / 16 / 81)\end{array}$ & 3540 & & SNL(?) & $?$ & $?$ & GE & $\begin{array}{l}\text { PA by } \\
\text { WESTING. }\end{array}$ \\
\hline ERDA-9 $* * x$ & $\begin{array}{l}\text { T21S R31E Sec } 20 * \\
265.32 \mathrm{FSL}, 180.40 \mathrm{FEL} \\
(6 / 26 / 76)\end{array}$ & 3408.86 & $*$ & SNL & 2875 & $\begin{array}{l}\text { USGS } \\
2 / 27 / 76 \\
\text { SEO } \\
6 / 21 / 76\end{array}$ & GE & $\begin{array}{l}\text { HSC \& } \\
\text { HMW }\end{array}$ \\
\hline ERDA-10 & $\begin{array}{l}\text { T23S R3OE Sec } 34 \\
200 \text { FNL, 2327 FEI } \\
(10 / 14 / 77)\end{array}$ & 3371.2 & & SNL & 4418 & $?$ & $G E$ & $\begin{array}{c}\text { PA (y) } \\
10 / 14 / 77\end{array}$ \\
\hline$\frac{\text { CABIN *:* }}{\underline{B A B Y-1}}$ & $\begin{array}{l}\text { T23S R31E Sec } 5 * \\
1993 \text { FNL, 2011 FEL } \\
(8 / 30 / 83)\end{array}$ & 3327.27 & $*$ & DOE & 4291 & $?$ & $\begin{array}{l}\text { Oil \& } \\
\text { Gas Well }\end{array}$ & $\begin{array}{l}\text { HSC \& } \\
\text { HMW }\end{array}$ \\
\hline$D-268 * x$ & $\begin{array}{l}\text { T22S R3OE Sec } 35 \div \\
716.5 \text { FSL, } 766.1 \text { FEI } \\
(8 / 29 / 84)\end{array}$ & 3278.54 & $*$ & SNL & ? & $?$ & $\begin{array}{l}\text { Potash } \\
\text { Test }\end{array}$ & HMT \\
\hline DOE-1 $x-x$ & $\begin{array}{l}\text { T22S R3IE Sec } 28 * \\
179.1 \text { FSL, } 610.5 \mathrm{FEL} \\
(7 / 28 / 82)\end{array}$ & 3465.09 & $*$ & WEST. & 4057 & $?$ & $\begin{array}{l}\text { GE \& } \\
\text { HTH }\end{array}$ & $\begin{array}{l}\text { HSC \& } \\
\text { HMW }\end{array}$ \\
\hline DOE-2 *x & $\begin{array}{l}\text { T22S R31E Sec } 8 \div \\
697.71 \mathrm{FSL}, 121.83 \mathrm{FEL} \\
(6 / 14 / 85)\end{array}$ & 3418.35 & $\star$ & SNL & 4325 & $\frac{\text { SEO \#\# }}{\frac{0.08 .1467}{6 / 14 / 84}}$ & $\begin{array}{l}\text { GE \& } \\
\text { HTH }\end{array}$ & $\begin{array}{l}\text { HSC \& } \\
\text { HHMW }\end{array}$ \\
\hline WIPP - II & $\begin{array}{l}\text { T22S R31E Sec } 9 * \\
708.89 \text { FNL, } 297.96 \text { FWL } \\
(3 / 14 / 78)\end{array}$ & 3426.07 & * & SNL & 3580 & $\frac{\text { SEO \#\# }}{\frac{0.08 .994}{12 / 15 / 77}}$ & $G E$ & $\begin{array}{l}\text { PA by } \\
\text { DOE? }\end{array}$ \\
\hline WIPP $-12 * *$ & $\begin{array}{l}\text { T22S R3IE Sec } 27 * \\
147.9 \mathrm{FSL}, 83.9 \mathrm{FEL} \\
(1 / 01 / 82)\end{array}$ & 3471.30 & $*$ & WEST.? & 3927 & ? & $G E$ & $\begin{array}{l}\text { HSC \& } \\
\text { HMW }\end{array}$ \\
\hline$\frac{\text { WIPP }-13 * *}{2563.6}$ & $\begin{array}{l}\text { T22S R31E Sec } 17 * \\
67 \text { FSL, } 1727.36 \text { FWL } \\
(10 / 05 / 79)\end{array}$ & 3405.43 & * & SNL & 3856 & $\frac{\text { SEO 輩 }}{\frac{0.08 .1182}{7 / 17 / 79}}$ & $G E$ & $\begin{array}{l}\text { HSC \& } \\
\text { HMW }\end{array}$ \\
\hline
\end{tabular}














\begin{tabular}{|c|c|c|c|c|c|c|c|c|}
\hline le No. & DATE OF COMPLETION & Elevation & & $\frac{\text { Legal }}{\text { Operator }}$ & $\frac{\text { Total }}{\text { Depth }}$ & $\frac{\text { Permit }}{\text { No. (SEO) }}$ & Purpose & $\frac{\text { Hole }}{\text { status }}$ \\
\hline$\underline{H-3 b 2 x x}$ & $\begin{array}{l}\text { T22S R31E Sec } 29 * \\
2122.09 \mathrm{FSI}, 231.36 \text { FEI } \\
\quad(11 / 11 / 83)\end{array}$ & 3389.33 & * & SNL & 725 & $\frac{\frac{\text { SEO \#\# }}{0.08 .991 ?}}{8 / 18 / 78}$ & HTH & HMW \\
\hline$\underline{H-3 b 3 * *}$ & $\begin{array}{l}\text { T22S R3IE Sec } 29 * \\
?(1 / 30 / 84)\end{array}$ & 3388.17 & $*$ & SNL & 730 & $\frac{\frac{S E O \text { 棐 }}{0.08 .991 ?}}{8 / 18 / 78}$ & HTH & HMW \\
\hline$\frac{H-3 d x * t}{(H-3 b 4)}$ & $\begin{array}{l}\text { T22S R3IE Sec } 29 * \\
2063.5 \text { FSL, } 161.1 \text { FEL } \\
(4 / 15 / 87)\end{array}$ & 3387.18 & 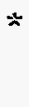 & SNL & 554 & $\frac{\frac{\text { SEO \#\# }}{8 / 08.991 ?}}{8 / 18 / 78}$ & $\mathrm{HTH}$ & HMW \\
\hline$\underline{H-4 a}$ & $\begin{array}{l}\text { T23S R3IE Sec } 5 * \\
\text { S47.60 FNI, } 716.28 \text { FWL } \\
(5 / 23 / 78)\end{array}$ & 3332.91 & $*$ & SNL & 532 & $\frac{\text { SEO \# }}{\frac{0.08 .1153}{4 / 24 / 78}}$ & HTH & HMW \\
\hline$H-4 b * x$ & $\begin{array}{l}\text { T23S R31E Sec } S * \\
\text { 500.58 FNL, } 629.14 \text { FWL } \\
(5 / 15 / 78)\end{array}$ & 3332.76 & $*$ & SNI & 529 & $\frac{\frac{\text { SEO 非 }}{0.08 .1154}}{4 / 24 / 78}$ & HTH & HMW \\
\hline$\frac{\mathrm{H}-4 \mathrm{c} \pm \mathrm{x}}{\tan }$ & $\begin{array}{l}\text { T23S R31E Sec S } * \\
447.09 \text { FNL, } 714.11 \text { FWI } \\
(5 / 09 / 78)\end{array}$ & 3333.54 & $\star$ & SNL & 661 & $\frac{\frac{\text { SEO \# }}{0.08 .1152}}{4 / 24 / 78}$ & $\mathrm{HTH}$ & HMW \\
\hline$\underline{H-5 a} \dot{x}$ & $\begin{array}{l}\text { T22S R31E Sec } 15 * \\
1095.30 \mathrm{FNL}, 187.52 \mathrm{FEI} \\
(6 / 20 / 78)\end{array}$ & 3506.15 & $*$ & SNL & 930 & $\frac{\frac{\text { SEO 輩 }}{0.08 .1159}}{5 / 11 / 78}$ & HTH & HMW \\
\hline$\underline{H-5 b}$ & $\begin{array}{l}\text { T22S R31E Sec } 15 \div \\
1010.03 \text { FNL, } 240.05 \text { FEL } \\
\quad(6 / 13 / 78)\end{array}$ & 3505.97 & * & SNL & 925 & $\frac{\frac{\text { SEO 非 }}{0.08 .1160}}{5 / 11 / 78}$ & HTH & HMW \\
\hline$\underline{H-5 c * x}$ & $\begin{array}{l}\text { T22S R31E Sec } 15 * \\
\text { I006.82 FNL, } 138.21 \text { FEL } \\
\quad(6 / 03 / 78)\end{array}$ & 3506.37 & $\dot{x}$ & SNL & 1076 & $\frac{\frac{\text { SEO 棐 }}{0.08 .1161}}{5 / 11 / 78}$ & HTH & HMW \\
\hline$\underline{H-6 a}$ & $\begin{array}{l}\text { T229 R31E Sec } 18 * \\
285.10 \text { FNL, } 270.55 \text { FWL } \\
(7 / 11 / 78)\end{array}$ & 3347.16 & $*$ & SNL & 637 & 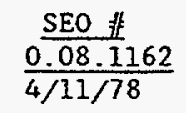 & HTH & HMW \\
\hline$H-6 b * x$ & $\begin{array}{l}\text { T22S R31E Sec } 18 \div \\
197.40 \mathrm{FNL}, 319.10 \mathrm{FWL} \\
(7 / 05 / 78)\end{array}$ & 3347.57 & $*$ & SNL & 640 & $\frac{\frac{\text { SEO 輩 }}{0.08 .1163}}{4 / 11 / 78}$ & HTH & HMW \\
\hline$H-6 c * t$ & $\begin{array}{l}\text { T22S R31E Sec } 18 \div \\
282.65 \mathrm{FNL}, 370.69 \mathrm{FWL} \\
(6 / 20 / 78)\end{array}$ & 3347.93 & $*$ & SNL & 741 & $\frac{\frac{\text { SEO 棐 }}{0.08 .1164}}{4 / 11 / 78}$ & HTH & HMW \\
\hline$\underline{H-7 a *}$ & $\begin{array}{l}\text { T23S R30E Sec } 14 * \\
\text { 2498.62 FNL, 2495.84 FWL } \\
(10 / 18 / 79)\end{array}$ & 3163.55 & * & SNL & 154 & $\frac{\frac{\text { SEO 非 }}{0.08 \cdot 1271}}{6 / 20 / 79}$ & HTH & HMW \\
\hline $\mathrm{H}-7 \mathrm{~b} \star \star \star$ & $\begin{array}{l}\text { T23S R31E Sec } 14 * \\
2568.75 \text { FNL, } 2567.49 \text { FWL } \\
(9 / 18 / 78)\end{array}$ & 3163.63 & * & SNL & 286 & $\frac{\frac{\text { SEO \#\# }}{0.08 .1272}}{6 / 20 / 79}$ & HTH & HMW \\
\hline
\end{tabular}




\begin{tabular}{|c|c|c|c|c|c|c|c|c|}
\hline iole No. & DATE OF COMPLETION & Elevation & & $\frac{\text { Legal }}{\text { perator }}$ & $\frac{\text { Total }}{\text { Depth }}$ & $\frac{\text { Permit }}{\text { No.(SEO })}$ & $\begin{array}{l}\text { Hole } \\
\text { Purpose }\end{array}$ & Sole \\
\hline$\underline{H}-7 b 2 * x$ & $\begin{array}{c}\text { T23S R31E Sec } 14 * \\
? \\
(9 / 23 / 83)\end{array}$ & 3163.95 & $*$ & SNL & 295 & $\frac{\text { SEO \#\# }}{?}$ & HTH & HMW \\
\hline $\mathrm{H}-7 \mathrm{c} \approx$ & $\begin{array}{l}\text { T23S R31E Sec } 14 * \\
2595.47 \mathrm{FNL}, 2471.05 \mathrm{FWL} \\
(11 / 02 / 79)\end{array}$ & 3163.48 & $*$ & SNL & 420 & $\frac{\frac{\text { SEO \#\# }}{0.08 .1273}}{6 / 20 / 79}$ & HTH & HMW \\
\hline$H-8 a \neq$ & $\begin{array}{l}\text { T24S R30E Sec } 23 * \\
19 E 3.43 \text { FNL, } 1481.66 \text { FEL } \\
(9 / 18 / 79)\end{array}$ & $3433.0 *$ & & SNL & 505 & $\frac{\frac{\text { SEO \#\# }}{6 / 08.1274}}{6 / 20 / 79}$ & HTH & HMW \\
\hline$\underline{H-8 b}+2=$ & $\begin{array}{l}\text { T24S R30E Sec } 23 * \\
\text { 1998.17 FNL, } 1401.76 \mathrm{FEL} \\
(8 / 12 / 79)\end{array}$ & $3433.8 *$ & & SNL (1) & 624 & $\frac{\frac{\text { SEO ff }}{0.08 .1275}}{6 / 20 / 79}$ & $\mathrm{HTH}$ & $\begin{array}{l}\text { Transf. } \\
\text { to owner }\end{array}$ \\
\hline $\mathrm{H}-8 \mathrm{c}=-\mathrm{s}$ & $\begin{array}{l}\text { T24S R30E Sec } 23 * \\
2062.22 \text { FNL, } 1466.04 \text { FEL } \\
(8 / 06 / 79)\end{array}$ & 3433.00 & 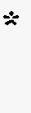 & SNL & 808 & $\frac{\frac{\text { SEO \# }}{0.08 .1276}}{6 / 20 / 79}$ & HTH & HMW \\
\hline $\mathrm{H}-9 a \pm=$ & $\begin{array}{l}\text { T24S R31E Sec } 4 * \\
2395.58 \mathrm{FNL}, 142.55 \text { FWI } \\
\quad(4 / 23 / 84)\end{array}$ & $\pi^{3405.4 *}$ & & SNL & 692 & $\frac{\frac{\text { SEO \#传 }}{0.08 \cdot 1277}}{6 / 20 / 79}$ & HTH & $\mathrm{HMW}$ \\
\hline$h-9 b \div$ & $\begin{array}{l}\text { T24S R3IE Sec } 4: \\
2395.58 \mathrm{FWL}, 242.55 \mathrm{FW} \\
(8 / 28 / 79)\end{array}$ & $\bar{n}^{340.5 .6 *}$ & & SNL & 708 & $\frac{\frac{\text { SEO 輩 }}{6 / 08.1278}}{6 / 20 / 79}$ & $\mathrm{HTH}$ & HMW \\
\hline $\mathrm{H}-9 \mathrm{c} \approx$ & $\begin{array}{l}\text { T24S R3iE Sec } 4 * \\
2482.18 \text { FNL, } 192.55 \text { FWI } \\
\quad(9 / 24 / 79)\end{array}$ & $\pi^{3405.9 *}$ & & SNL & 816 & $\frac{\frac{\text { SEO 非 }}{0.08 .1279}}{6 / 20 / 79}$ & HTH & HMW \\
\hline $\mathrm{H}-10 \mathrm{a}= \pm \frac{\mathrm{t}}{\mathrm{t}}$ & $\begin{array}{l}\text { T24S R31E Sec } 20 * \\
431.0 \text { FSL, } 2064.9 \text { FEL } \\
(8 / 26 / 79)\end{array}$ & 3686.52 & 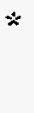 & SNI & 1318 & $\frac{\frac{\text { SEO \# }}{0.08 .1280}}{6 / 20 / 79}$ & HTH & HMW \\
\hline$\underline{\mathrm{H}-10 \mathrm{~b}: 2}$ & $\begin{array}{l}\text { T24S R3IE Sec } 20 \approx \\
481.0 \text { FSL, } 1978.3 \text { FEL } \\
\quad(10 / 13 / 79)\end{array}$ & 3687.01 & $\star$ & SNL & 1398 & $\frac{\frac{\text { SEO \#\# }}{0.08 .1281}}{6 / 20 / 79}$ & HTH & HMW \\
\hline $\mathrm{H}-10 \mathrm{C} \approx \mathrm{C}$ & $\begin{array}{l}\text { I24S R31E Sec } 20 * \\
381.0 \mathrm{FSL}, 1978.3 \mathrm{FEL} \\
\quad(8 / 20 / 79)\end{array}$ & 3686.88 & $\therefore$ & SNL & 1550 & $\frac{\frac{\text { SEO \#\# }}{0.08 .1282}}{6 / 20 / 79}$ & HTH & HMW \\
\hline$\underline{H-11 b I * x}$ & $\begin{array}{l}\text { T22S R31E Sec } 33 * \\
1510.69 \mathrm{FSL}, 173.91 \text { FEI } \\
\quad(9 / 2 / 83)\end{array}$ & $I^{3411.0}$ & & SNL & 785 & $\frac{\frac{\text { SEO \# }}{0.08 .1462}}{11 / 21 / 83}$ & HTH & HMW \\
\hline $\mathrm{H}-11 \mathrm{~b} 2 * x$ & $\begin{array}{l}\text { T22S R31E Sec } 33 * \\
\text { I436.16 FSL, } 168.62 \text { FEI } \\
\quad(11 / 28 / 83)\end{array}$ & 3411.2 & & SNL & 776 & $\frac{\frac{\text { SEO \# }}{0.08 .1462}}{11 / 21 / 83}$ & HTH & HMW \\
\hline$\underline{H-11 b 3 * *}$ & $\begin{array}{l}\text { T22S R31E Sec } 33 * \\
2499.2 \mathrm{FSL}, 106.6 \mathrm{FEL} \\
(1 / 10 / 84)\end{array}$ & 3412.07 & * & SNL & 789 & 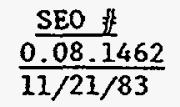 & HTH & HMW \\
\hline
\end{tabular}




\begin{tabular}{|c|c|c|c|c|c|c|c|}
\hline iole No. & LOCATION \& & Flevation & $\frac{\text { Legal }}{\text { Operator }}$ & Total & $\frac{\text { Permit }}{\left.(S)^{\prime}\right)}$ & Hole & $\frac{\text { Hole }}{\text { statu: }}$ \\
\hline$\underline{H-11 b 4 \quad x-x}$ & $\begin{array}{l}\text { T22S R31E } \operatorname{Sec} 33 * \\
\text { 1516.5 FSL, } 315.2 \mathrm{FEL} \\
(3 / 17 / 88)\end{array}$ & $3410.1 \dot{x}$ & SNL & 765 & $\frac{\frac{\text { SEO \# }}{0.08 .1474}}{12 / 29 / 87}$ & HTH & HMW \\
\hline $\mathrm{H}-12 \times 2$ & $\begin{array}{l}\text { T23S R31E Sec } 15 * \\
26.5 \text { FNL, } 94.4 \text { FEL } \\
(10 / 31 / 83)\end{array}$ & 3425.98 & SNL & 1001 & $\frac{\text { SEO 棐 }}{\frac{0.08 .1463}{1 / 21 / 83}}$ & HTH & HMW \\
\hline$\underline{H-14 * 2}$ & $\begin{array}{l}\text { T22S R3IE Sec } 29 \neq \\
369.5 \text { FSL, 564.7 FWL } \\
\quad(10 / 23 / 86)\end{array}$ & $3345.48=$ & SNL & 589 & $\frac{\frac{\text { SEO \#侻 }}{0.08 .1469}}{9 / 12 / 86}$ & HTH & HMW \\
\hline $\mathrm{H}-15 \div-\mathrm{x}$ & $\begin{array}{l}\text { T22S R3IE Sec } 28 * \\
92.6 \text { FNL, } 170.6 \text { FEL } \\
(11 / 14 / 86)\end{array}$ & $3480.2 *$ & SNL & 900 & 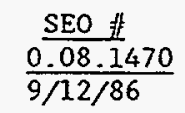 & HTH & HMW \\
\hline $\mathrm{H}-16$ 次 & $\begin{array}{l}\text { T22S R31E Sec } 20 \div \\
1109.3 \mathrm{FSL}, 1234.0 \mathrm{FEL} \\
(8 / 18 / 87)\end{array}$ & $3409.6 *$ & SNL & 851 & $\frac{\frac{\text { SEO H }}{0.08 .1471}}{6 / 26 / 87}$ & $\mathrm{HTH}$ & HMW \\
\hline $\mathrm{H}-17 \times 2$ & $\begin{array}{l}\text { T23S R31E Sec } 3 \div \\
1463.2 \mathrm{FSL}, 991.8 \mathrm{FWL} \\
\quad(11 / 04 / 87)\end{array}$ & $3384.01=$ & $\doteqdot \quad$ SNL & 880 & $\frac{\frac{\text { SEO 非 }}{0.08 .1472}}{8 / 28 / 87}$ & HTH & HMV \\
\hline$\underline{\mathrm{H}-18 * 2}$ & $\begin{array}{l}\text { T22S R3IE Sec } 20 \div \\
968.4 \text {. FNL, } 447.5 \mathrm{FWI} \\
(11 / 18 / 87)\end{array}$ & $3413.36=$ & $\approx$ SNL & 840 & $\frac{\frac{\text { SEO \# }}{0.08 .1473}}{8 / 28 / 87}$ & HTH & HMW \\
\hline
\end{tabular}




\section{Sandia National \\ Laboratories}

Albuquerque, New Mexico 87185-1326

date: October 26, 1994

to: $\quad$ Leslie R. Hill, MS-1341

from: Jerry W. Mercer, Dept. 9333, MS-1156

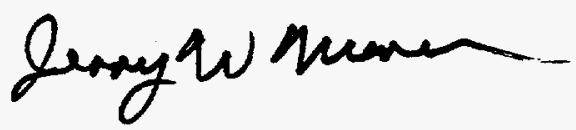

subject: $\quad$ WIPP Surface Boreholes - H-3 Hydropad

The $\mathrm{H}-3 \mathrm{~b} 2$ and $\mathrm{H}-3 \mathrm{~b} 3$ surface boreholes were drilled for hydrologic studies in the Culebra Member of the Rustler and the H-3d borehole was drilled for hydrologic evaluation of the overlying Dewey Lake Redbeds. Your difficulties in obtaining the drilling information you are requesting stem from the fact that standard SNL basic data reports were not completed for these boreholes. Attached is a summary of information regarding these boreholes from field notes I compiled during the drilling activities. Additional information can be found in: Pearson et al. 1987, "Preliminary Design for a Sorbing Tracer Test in the Culebra Dolomite at the H-3 Hydropad at the Waste Isolation Pilot Plant (WIPP) Site," SAND86-7177.

Please feel free to contact me if you have additional questions about these boreholes or if I might be of further assistance in any other issue arising during the completion of your report.

cc: Richard Aguilar, MS-1326 


\title{
Sandia National Laboratories
}

Albuquerque. New Mexico 87185-1326

date: July 18, 1996

to: Leslie R. Hill, MS-1341

\author{
Geray whern \\ from: Jerry W. Mercer, Dept. 9333, MS-1156 \\ subject: H-19 Hydropad Borehole Information
}

I have reviewed the information on the $\mathrm{H}-19$ hydropad boreholes $(\mathrm{H}-19 \mathrm{~b} 0, \mathrm{H}$ 19b1, H-19b2, H-19b3, H-19b4, H-19b5, H-19b5 [deepened], H-19b6, H-19b6 [deepened], and H-19b7) outlined in pages 24 through 27 of the July 1, 1996 draft of the report entitled: "Condensed Listing of Surface Boreholes at the Waste Isolation Pilot Plant Project through 31 December 1995"; authors: Hill, L. R., R. Aguilar, J. W. Mercer, and G. Newman. All of the H-19 hydropad borehole information listed in the report, including borehole ID, drilling start/end dates, purpose, status, township/range/section, section X \& $Y$ coordinates, X \& Y state-plane coordinates, surface elevation, total depth, core diameters, sampling intervals, and comments, is accurate and consistent with the information recorded in my data log books and field notes. These data are not subject to future change because they are factual data. Furthermore, all of this information is consistent with the data presented in our draft report for the $\mathrm{H}-19$ hydropad drilling activities. This draft report is currently in the final review process for publication in the form of a SAND report.

cc: Richard Aguilar, MS-1326

Gretchen Newman, GRAM, Inc.

Richard Thompson, MS-1341

Mel Marietta, MS-1395 
Page intentionally left blank.

A-16 
APPENDIX B: AS-BUILT ELEMENTS OF THE FOUR WIPP SHAFTS

B-1 
Page intentionally left blank.

B-2 


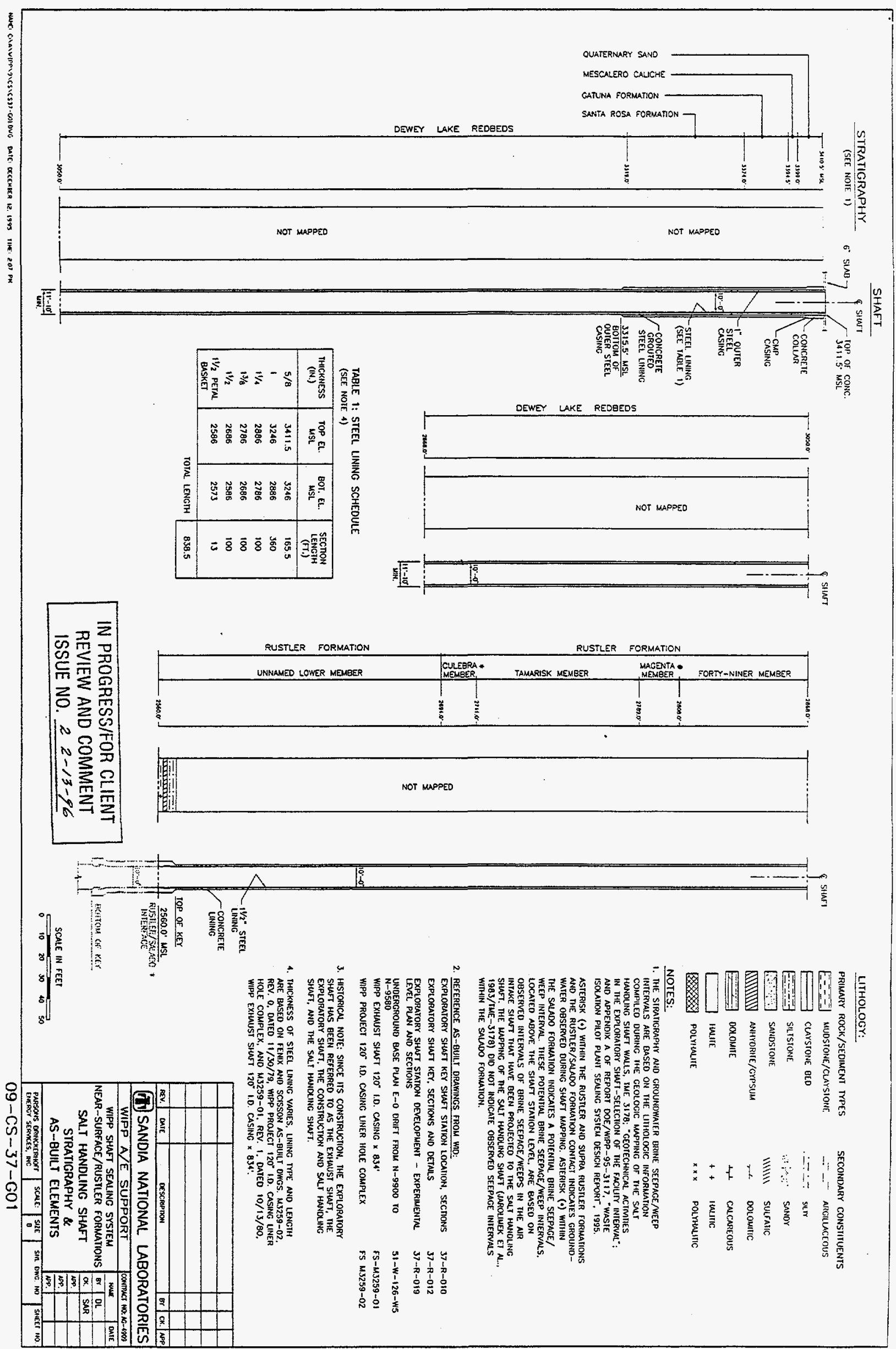




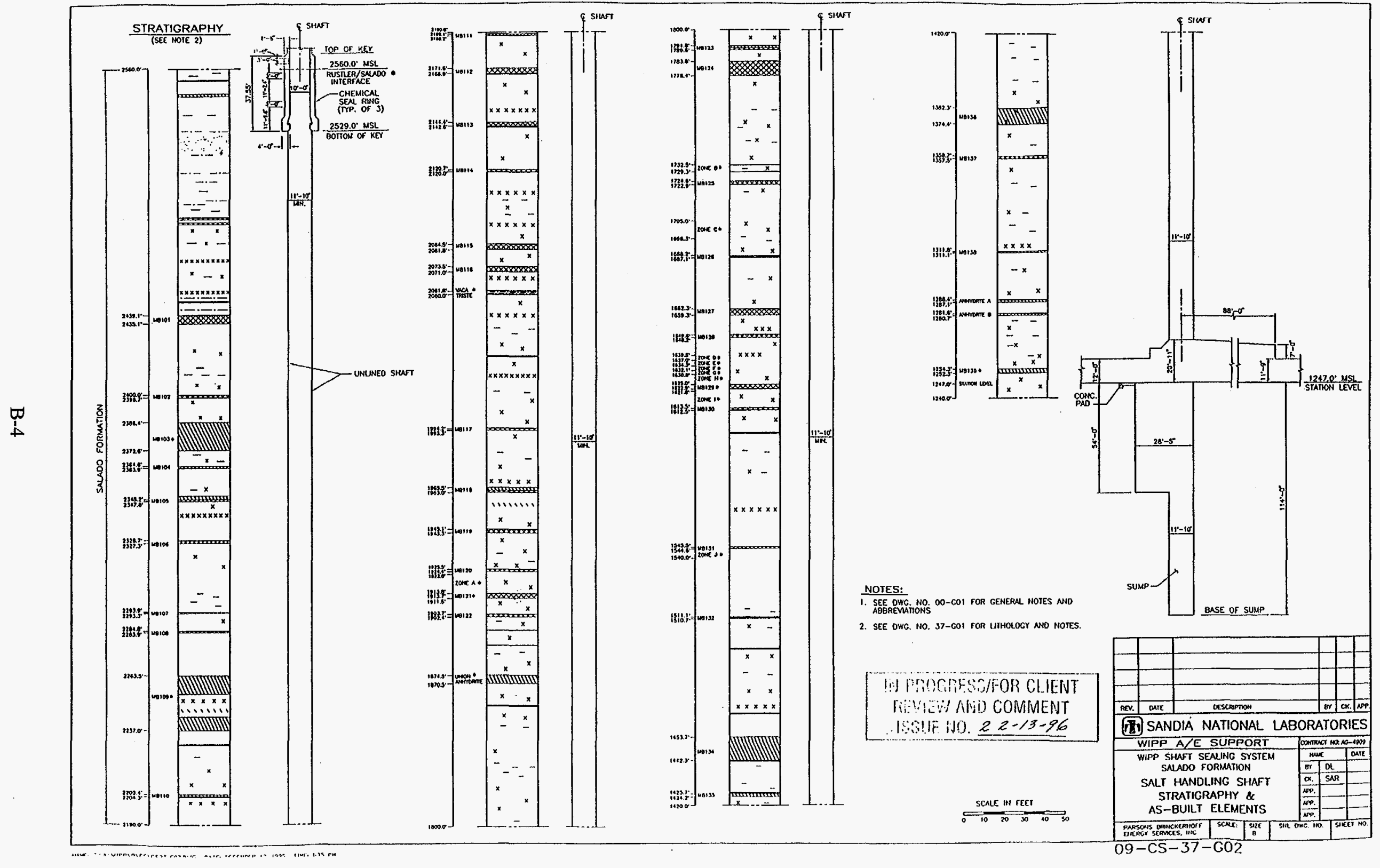






B-5 


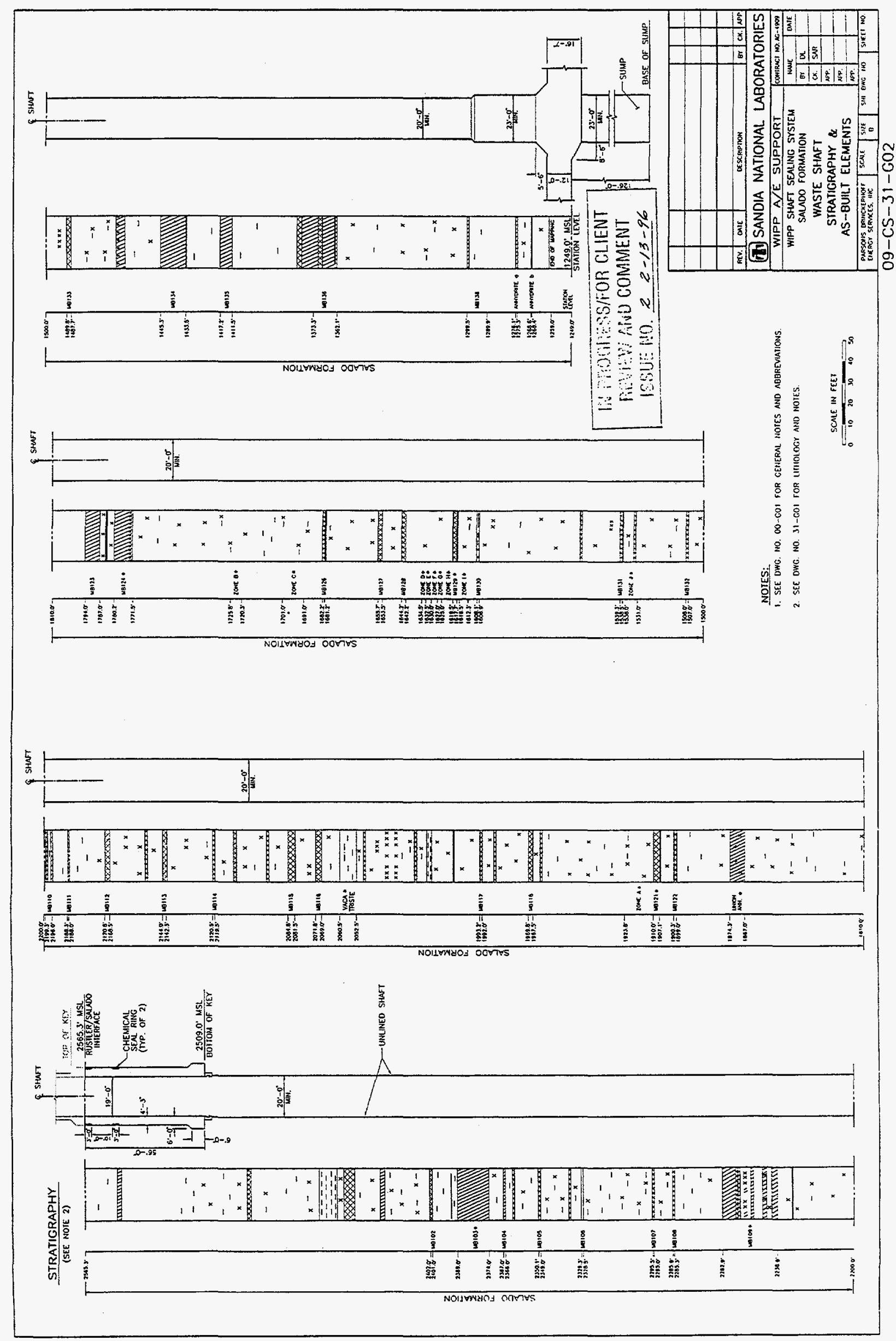




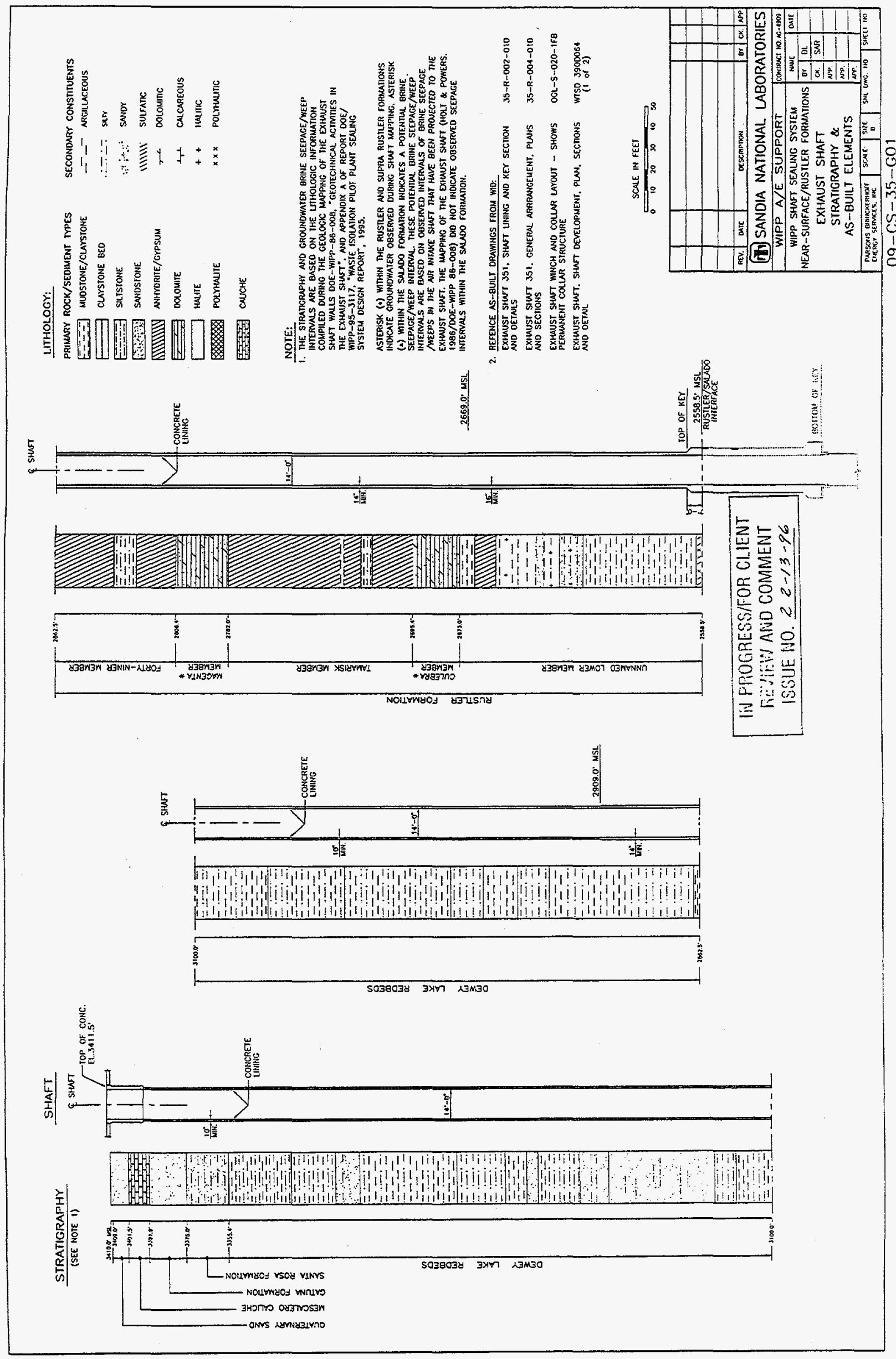









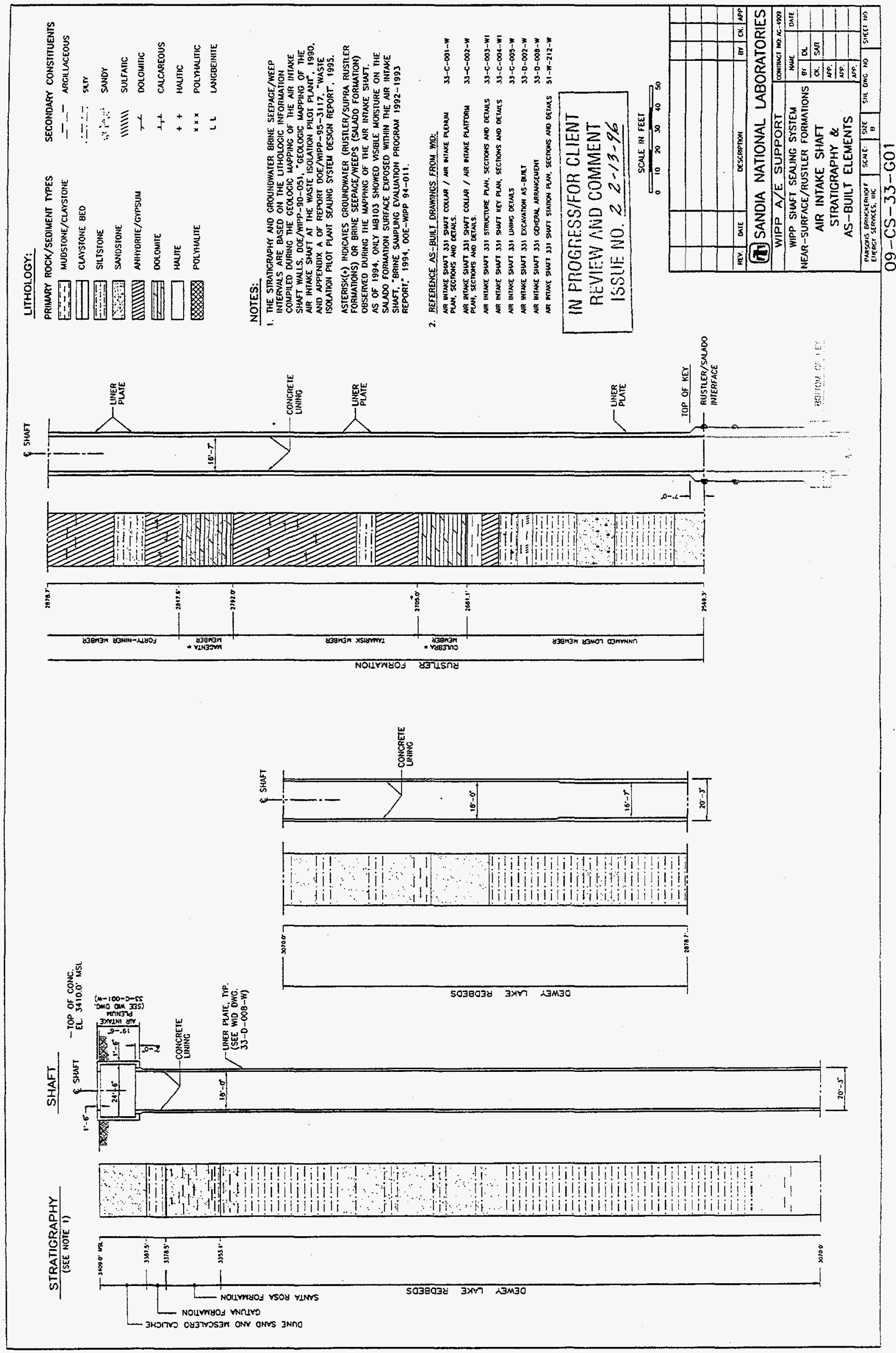




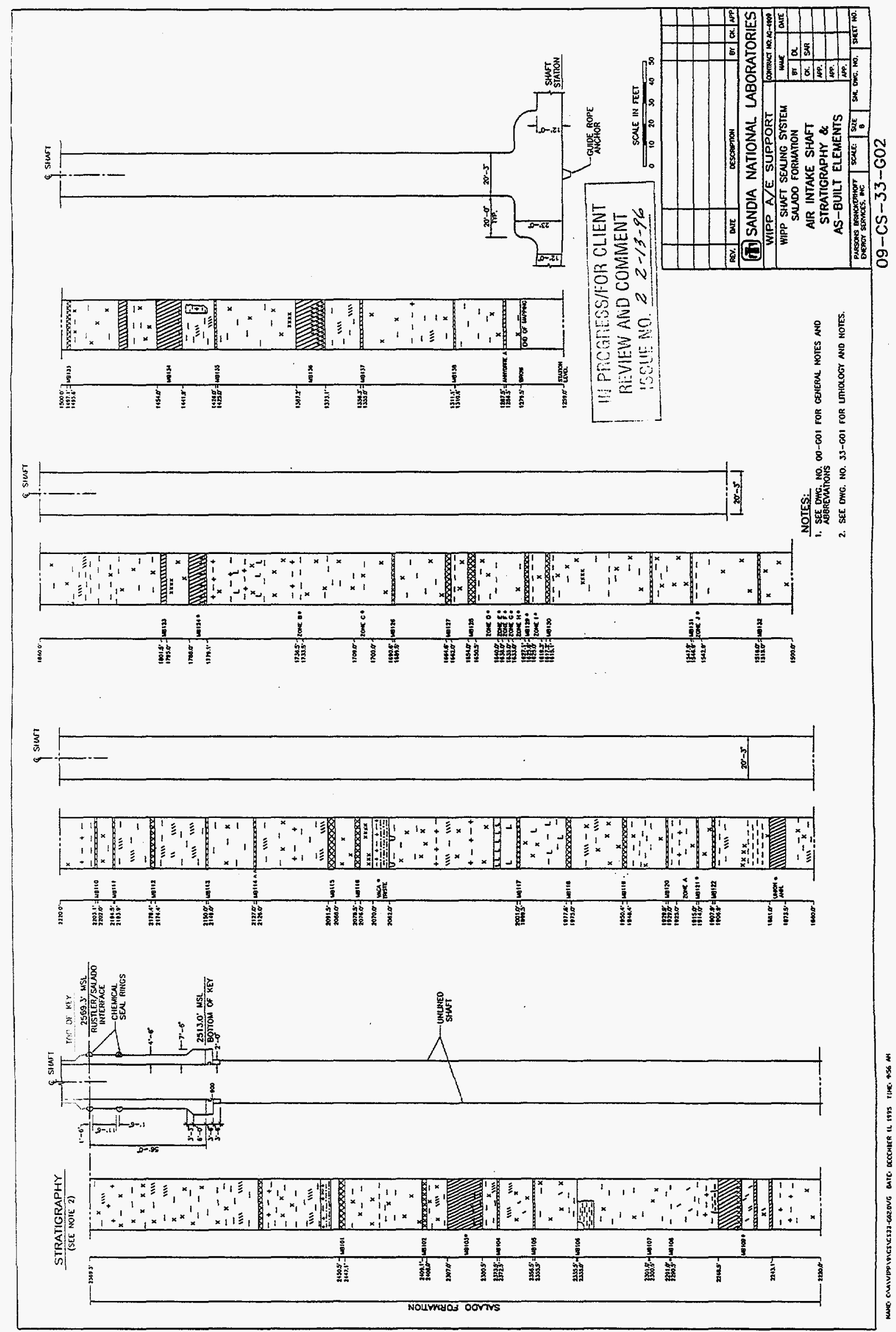

B-10 


\section{WIPP \\ UC721 - DISTRIBUTION LIST \\ SAND96-1960}

\section{Federal Agencies}

US Department of Energy (4)

Office of Civilian Radioactive Waste Mgmt.

Attn: Deputy Director, RW-2

Acting Director, RW-10

Office of Human Resources \& Admin.

Director, RW-30

Office of Program Mgmt. \& Integ.

Director, RW-40

Office of Waste Accept., Stor., \& Tran.

Forrestal Building

Washington, DC 20585

Attn: Project Director

Yucca Mountain Site Characterization Office Director, RW-3

Office of Quality Assurance

101 Convention Center Drive, Suite \#P-110

Las Vegas, NV 89109

US Department of Energy

Albuquerque Operations Office

Attn: National Atomic Museum Library

P.O. Box 5400

Albuquerque, NM 87185-5400

US Department of Energy

Research \& Waste Management Division

Attn: Director

P.O. Box E

Oak Ridge, TN 37831

US Department of Energy (5)

Carlsbad Area Office

Attn: G. Dials

D. Galbraith

M. McFadden

R. Lark

J. A. Mewhinney

P.O. Box 3090

Carlsbad, NM 88221-3090

US Department of Energy

Office of Environmental Restoration and

Waste Management

Attn: J. Lytle, EM-30

Forrestal Building

Washington, DC 20585-0002
US Department of Energy (3)

Office of Environmental Restoration and Waste Management

Attn: M. Frei, EM-34, Trevion II

Washington, DC 20585-0002

US Department of Energy

Office of Environmental Restoration and

Waste Management

Attn: S. Schneider, EM-342, Trevion II

Washington, DC 20585-0002

US Department of Energy (2)

Office of Environment, Safety \& Health

Attn: C. Borgstrom, EH-25

R. Pelletier, EH-231

Washington, DC 20585

US Department of Energy (2)

Idaho Operations Office

Fuel Processing \& Waste Mgmt. Division

785 DOE Place

Idaho Falls, ID 83402

US Environmental Protection Agency (2)

Radiation Protection Programs

Attn: M. Oge

ANR-460

Washington, DC 20460

Boards

Defense Nuclear Facilities Safety Board

Attn: D. Winters

625 Indiana Ave. NW, Suite 700

Washington, DC 20004

Nuclear Waste Technical Review Board (2)

Attn: Chairman S. J. S. Parry

1100 Wilson Blvd., Suite 910

Arlington, VA 22209-2297 


\section{State Agencies}

Attorney General of New Mexico P.O. Drawer 1508

Santa Fe, NM 87504-1508

Environmental Evaluation Group (3)

Attn: Library

7007 Wyoming NE

Suite F-2

Albuquerque, NM 87109

NM Energy, Minerals, and Natural Resources Department

Attn: Library

2040 S. Pacheco

Santa Fe, NM 87505

NM Environment Department (3)

Secretary of the Environment

Attn: Mark Weidler

1190 St. Francis Drive

Santa Fe, NM 87503-0968

NM Bureau of Mines \& Mineral Resources

Socorro, NM 87801

NM Environment Department

WIPP Project Site

Attn: P. McCasland

P.O. Box 3090

Carlsbad, NM 88221

\section{Laboratories/Corporations}

Battelle Pacific Northwest Laboratories Attn: R. E. Westerman, MSIN P8-44 Battelle Blvd.

Richland, WA 99352

INTERA, Inc.

Attn: G. A. Freeze

1650 University Blvd. NE, Suite 300

Albuquerque, NM 87102

INTERA, Inc.

Attn: J. F. Pickens

6850 Austin Center Blvd., Suite 300

Austin, TX 78731

INTERA, Inc.

Attn: W. Stensrud

P.O. Box 2123

Carlsbad, NM 88221
Los Alamos National Laboratory

Attn: B. Erdal, INC-12

P.O. Box 1663

Los Alamos, NM 87544

RE/SPEC, Inc

Attn: Angus Robb

4775 Indian School NE, Suite 300

Albuquerque, NM 87110-3927

RE/SPEC, Inc

Attn: J. L. Ratigan

P.O. Box 725

Rapid City, SD 57709

Tech Reps, Inc. (6)

Attn: J. Chapman (1)

Loretta Robledo (2)

$\mathrm{R}$. Jones (1)

T. Peterson (1)

M. Minahan (1)

5000 Marble NE, Suite 222

Albuquerque, NM 87110

Westinghouse Electric Corporation (5)

Attn: Library

J. Epstein

J. Lee

B. A. Howard

R. Kehrman

P.O. Box 2078

Carlsbad, NM 88221

S. Cohen \& Associates

Attn: Bill Thurber

1355 Beverly Road

McLean, VA 22101

GRAM

Attn: G. Newman

8500 Menaul NE

Albuquerque, NM 87112

LATA

Attn: Leo. W. Scully

2400 Louisiana Blvd NE

Albuquerque, NM 87110 


\section{National Academy of Sciences, WIPP Panel}

Howard Adler

Oxyrase, Incorporated

7327 Oak Ridge Highway

Knoxville, TN 37931

Bob Andrews

Board of Radioactive Waste Management

GF456

2101 Constitution Ave.

Washington, DC 20418

Rodney C. Ewing

Department of Geology

University of New Mexico

Albuquerque, NM 87131

Charles Fairhurst

Department of Civil and Mineral Engineering

University of Minnesota

500 Pillsbury Dr. SE

Minneapolis, MN 55455-0220

B. John Garrick

PLG Incorporated

4590 MacArthur Blvd., Suite 400

Newport Beach, CA 92660-2027

Leonard F. Konikow

US Geological Survey

431 National Center

Reston, VA 22092

Carl A. Anderson, Director

Board of Radioactive Waste Management

National Research Council

HA 456

2101 Constitution Ave. NW

Washington, DC 20418

Christopher G. Whipple

ICF Kaiser Engineers

1800 Harrison St., 7th Floor

Oakland, CA 94612-3430

John O. Blomeke

720 Clubhouse Way

Knoxville, TN 37909
Sue B. Clark

University of Georgia

Savannah River Ecology Lab

P.O. Drawer E

Aiken, SC 29802

Konrad B. Krauskopf

Department of Geology

Stanford University

Stanford, CA 94305-2115

Della Roy

Pennsylvania State University

217 Materials Research Lab

Hastings Road

University Park, PA 16802

David A. Waite

$\mathrm{CH}_{2} \mathrm{M}$ Hill

P.O. Box 91500

Bellevue, WA 98009-2050

Thomas A. Zordon

Zordan Associates, Inc.

3807 Edinburg Drive

Murrysville, PA 15668

\section{Universities}

University of New Mexico

Geology Department

Attn: Library

141 Northrop Hall

Albuquerque, NM 87131

University of Washington

College of Ocean \& Fishery Sciences

Attn: G. R. Heath

583 Henderson Hall, HN-15

Seattle, WA 98195

\section{Libraries}

Thomas Brannigan Library

Attn: D. Dresp

106 W. Hadley St.

Las Cruces, NM 88001

Government Publications Department

Zimmerman Library

University of New Mexico

Albuquerque, NM 87131 
New Mexico Junior College

Pannell Library

Attn: R. Hill

Lovington Highway

Hobbs, NM 88240

New Mexico State Library

Attn: N. McCallan

325 Don Gaspar

Santa Fe, NM 87503

New Mexico Tech

Martin Speere Memorial Library

Campus Street

Socorro, NM 87810

WIPP Public Reading Room

Carlsbad Public Library

$101 \mathrm{~S}$. Halagueno St.

Carlsbad, NM 88220

\section{Foreign Addresses}

Atomic Energy of Canada, Ltd.

Whiteshell Laboratories

Attn: B. Goodwin

Pinawa, Manitoba, CANADA ROE ILO

Francois Chenevier (2)

ANDRA

Route de Panorama Robert Schumann

B. P. 38

92266 Fontenay-aux-Roses, Cedex

FRANCE

Claude Sombret

Centre d'Etudes Nucleaires de la Vallee Rhone

CEN/VALRHO

S.D.H.A. B.P. 171

30205 Bagnols-Sur-Ceze, FRANCE

Commissariat a L'Energie Atomique

Attn: D. Alexandre

Centre d'Etudes de Cadarache

13108 Saint Paul Lez Durance Cedex

FRANCE

Bundesanstalt fur Geowissenschaften und

Rohstoffe

Attn: M. Langer

Postfach 510153

D-30631 Hannover, GERMANY
Bundesministerium fur Forschung und

Technologie

Postfach 200706

5300 Bonn 2, GERMANY

Institut fur Tieflagerung

Attn: K. Kuhn

Theodor-Heuss-Strasse 4

D-3300 Braunschweig, GERMANY

Gesellschaft fur Anlagen und Reaktorsicherheit (GRS)

Attn: B. Baltes

Schwertnergasse 1

D-50667 Cologne, GERMANY

Shingo Tashiro

Japan Atomic Energy Research Institute

Tokai-Mura, Ibaraki-Ken, 319-11

JAPAN

Netherlands Energy Research Foundation ECN

Attn: J. Prij

3 Westerduinweg

P.O. Box 1

1755 ZG Petten

THE NETHERLANDS

Svensk Karnbransleforsorjning $A B$

Attn: F. Karlsson

Project KBS (Karnbranslesakerhet)

Box 5864

S-102 48 Stockholm

SWEDEN

Nationale Genossenschaft fur die Lagerung

Radioaktiver Abfalle (2)

Attn: S. Vomvoris

P. Zuidema

Hardstrasse 73

CH-5430 Wettingen

SWTTZERLAND

AEA Technology

Attn: J. H. Rees

D5W/29 Culham Laboratory

Abington, Oxfordshire OX14 3DB

UNITED KINGDOM

AEA Technology

Attn: W. R. Rodwell

044/A31 Winfrith Technical Centre

Dorchester, Dorset DT2 8DH

UNTTED KINGDOM 
AEA Technology

Attn: J. E. Tinson

B4244 Hanwell Laboratory

Didcot, Oxfordshire OX11 ORA

UNITED KINGDOM

\section{Other}

Dr. Dennis W. Powers

H.G. 12

Box 87

Anthony, TX 79821

Dr. George B. Griswold

6813 Via Campestre

Las Cruces, NM 88005

Robert D. Statler

P.O. Box 1556

Dewey, AZ 86327

Paul D. Seward

9604 Merion Cir. NE

Albuquerque, NM 87111

Internal

\begin{tabular}{|c|c|c|}
\hline MS & Org. & \\
\hline 1324 & 6115 & P. B. Davies \\
\hline 1320 & 6831 & E. J. Nowak \\
\hline 1322 & 6121 & J. R. Tillerson \\
\hline 1328 & 6849 & D. R. Anderson \\
\hline 1328 & 6848 & H. N. Jow \\
\hline 1335 & 6801 & M. Chu \\
\hline 1341 & 6832 & J. T. Holmes \\
\hline 1395 & 6800 & L. Shephard \\
\hline 1395 & 6821 & M. Marietta \\
\hline 1395 & 6841 & V. H. Slaboszewicz \\
\hline 1337 & 6000 & W. D. Weart \\
\hline 1341 & 6822 & L R. Hill (2) \\
\hline 1326 & 6851 & R. Aguilar (2) \\
\hline 1156 & 9333 & J.W. Mercer (2) \\
\hline 1395 & 6821 & P. E. Sanchez \\
\hline 1328 & 6849 & M. Wallace \\
\hline 1328 & 6849 & D. M. Stoelzel \\
\hline 0750 & 6118 & S. J. Lambert \\
\hline 1345 & 6331 & R. V. Guzowski \\
\hline 1322 & 6121 & F.D. Hansen \\
\hline 1330 & 6811 & K. Hart (2) \\
\hline 1330 & 4415 & NWM Library (20) \\
\hline 9018 & $8940-2$ & Central Technical Files \\
\hline 0899 & 4414 & Technical Library (5) \\
\hline 0619 & 12630 & $\begin{array}{l}\text { Review and Approval Desk (2), } \\
\text { For DOE/OSTI }\end{array}$ \\
\hline
\end{tabular}

Distribution - 5 